\title{
Par Pond Refill Water Quality Sampling
}

by

J. W. Koch

Westinghouse Savannah River Company

Savannah River Site

Aiken, South Carolina 29808

F. D. Martin

H. M. Westburv

\section{DOE Contract No. DE-AC09-89SR18035}

This paper was prepared in connection with work done under the above contract number with the U.S. Department of Energy. By acceptance of this paper, the publisher and/or recipient acknowledges the U. S. Government's right to retain a nonexclusive, royalty-free license in and to any copyright covering this paper, along with the right to reproduce and to authorize others to reproduce all or part of the copyrighted paper. 


\section{Par Pond Refill Water Quality Sampling (U)}

J. W. Koch II, F. D. Martin, and H. M. Westbury

Westinghouse Savannah River Company

Savannah River Technology Center

Environmental Sciences Section

Aiken, SC 29808

August 1996

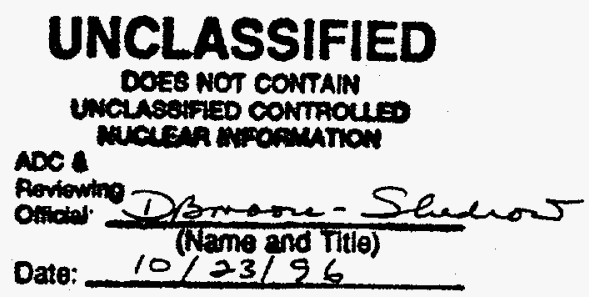

The information contained in this report was developed during the course of work with the U.S. Department of Energy under Contract No. DE-AC09-89SR18035. 


\section{DISCLAIMER}

This report was prepared as an account of work sponsored by an agency of the United States Government. Neither the United States Government nor any agency thereof, nor any of their employees, makes any warranty, express or implied, or assumes any legal liability or responsibility for the accuracy, completeness, or usefulness of any information, apparatus, product, or process disclosed, or represents that its use would not infringe privately owned rights. Reference herein to any specific commercial product, process, or service by trade name, trademark, manufacturer, or otherwise does not necessarily constitute or imply its endorsement, recommendation, or favoring by the United States Government or any agency thereof. The views and opinions of authors expressed herein do not necessarily state or reflect those of the United States Government or any agency thereof.

This report has been reproduced directly from the best available copy.

Available to DOE and DOE contractors from the Office of Scientific and Technical Information, P.O. Box 62, Oak Ridge, TN 37831; prices available from (615) 576-8401.

Available to the public from the National Technical Information Service, U.S. Department of Commerce; 5285 Port Royal Road, Springfield, VA 22161. 


\section{DISCLAIMER}

Portions of this document may be illegible electronic image products. Images are produced from the best available original document. 


\section{Table of Contents}

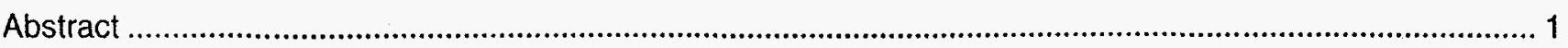

Introduction

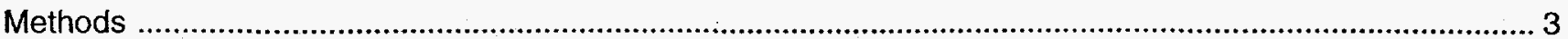

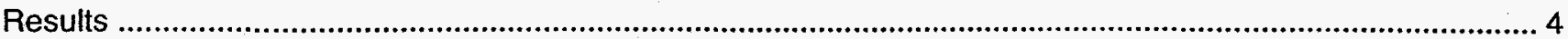

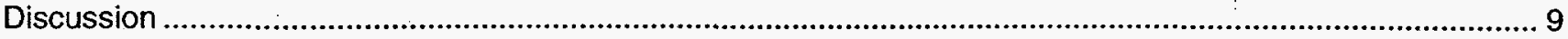

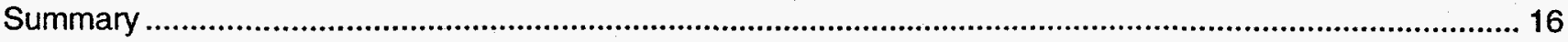

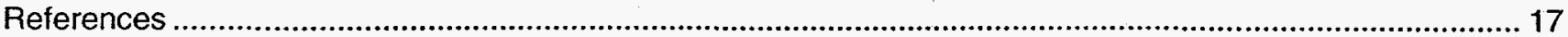

Appendix A Field Results Dissolved Oxygen and Temperature at Four Par Pond Locations, February-September 1995

Appendix B Analytical Results 


\section{List of Tables}

Table 1.

Universal Transverse Mercator coordinates for Par Pond water quality

sample locations

\section{List of Figures}

Figure 1.

Map of Par Pond indicating water quality sampling locations.

February-September 1995

Figure 2.

Rate of refill of Par Pond from 55 meters ( 181 feet) above mean sea level

to approximately 61 meters (200 feet) above mean sea level

Figure 3.

Dissolved oxygen concentrations through the water column at Station 1.

February-August 1995.

Figure 4.

Dissolved oxygen concentrations through the water column at Station 2.

February-August 1995

Figure 5.

Dissolved oxygen concentrations through the water column at Station 3.

February-August 1995

Figure 6.

Dissolved oxygen concentrations through the water column at Station 4.

February-August 1995

Figure 7.

Chlorophyll-a concentrations from surface (upper figure) and bottom

(lower figure) water samples at sample locations in Par Pond.

February-September 1995

Figure 8.

Nitrate concentrations from surface (upper figure) and bottom (lower figure) water samples at sample locations in Par Pond.

February-September 1995

Figure 9.

Ammonia concentrations from surface (upper figure) and bottom

(lower figure) water samples at sample locations in Par Pond.

February-September 1995

Figure 10.

Total Kjeldahl nitrogen concentrations from surface (upper figure) and bottom

(lower figure) water samples at sample locations in Par Pond.

February-September 1995 


\section{List of Figures (continued)}

Figure 11.

Orthophosphate concentrations from surface (upper figure) and bottom (lower figure) water samples at sample locations in Par Pond.

February-September 1995

Figure 12.

Total dissolved phosphorus concentrations from surface (upper figure) and

bottom (lower figure) water samples at sample locations in Par Pond.

February-September 1995 


\title{
Par Pond Refill Water Quality Sampling (U)
}

\author{
J. W. Koch, F. D. Martin, and H. M. Westbury \\ Westinghouse Savannah River Company \\ Savannah River Technology Center \\ Environmental Sciences Section \\ Aiken, SC 29808
}

\begin{abstract}
In February 1995, biweekly water sampling was initiated to monitor changes in water chemistry during the refilling of Par Pond to its full pool, approximately 61 meters ([m] 200 feet) above mean sea level. This sampling program was designed to measure and monitor parameters and constituents that could quickly indicate impending anoxia (oxygen depletion) or eutrophication (nutrient imbalance). Field measurements of dissolved oxygen and water temperature were taken at 1-m depth intervals throughout the water column at all locations. Samples for chemical analyses were taken from the surface and the bottom of the reservoir at the same locations as the field measurements. Laboratory analyses for nitrogen, phosphorus, and chlorophyll-a were performed.

Refilling Par Pond created the potential for the destabilization of the reservoir's nutrient balance caused by the decay of terrestrial plants that had previously grown on the exposed sediments and were now inundated by water. It was hoped that by refilling during the winter, when above-ground terrestrial biomass is low with little or no stored nutrients and aquatic biotic activity is minimal, any eutrophication or anoxia that could result from the sudden release of nutrients would be avoided.

This study was designed to document anoxia and its cause in the event that the anoxia caused a fish kill. However, no fish kill was observed during this study, and dissolved oxygen and nutrient concentrations generally remained within the range expected for southeastern reservoirs. Par Pond water quality monitoring will continue during the second summer after refill as the aquatic macrophytes become reestablished and nutrients in the sediments are released to the water column.
\end{abstract}

\section{Introduction}

Par Pond (Figure 1) was created in 1958 by impounding the headwaters of Lower Three Runs with an earthen embankment. Through 1963, Par Pond was utilized as a recirculating cooling reservoir for $\mathrm{P}$ and $\mathrm{R}$ Reactors at the Savannah River Site (SRS). Par Pond was used to cool P-Reactor effluents from 1964, when R Reactor was shutdown, until 1988, when P-Reactor operations were discontinued. Since the lake was created, makeup water has been pumped into Par Pond from the Savannah River to replace water lost by evapora- tion and seepage to maintain a constant water level in the reservoir.

During the spring of 1991 , a depression was discovered on the downstream slope of the Par Pond dam. The Par Pond water level was lowered from approximately 61 meters ([m] 200 feet) to approximately $55 \mathrm{~m}$ (181 feet) above mean sea level to repair the dam and to ensure the safety of downstream residents. The Par Pond drawdown exposed the contaminated sediments in the basin; consequently the exposed area was listed as a Comprehensive Environmental Response, Compensation, 


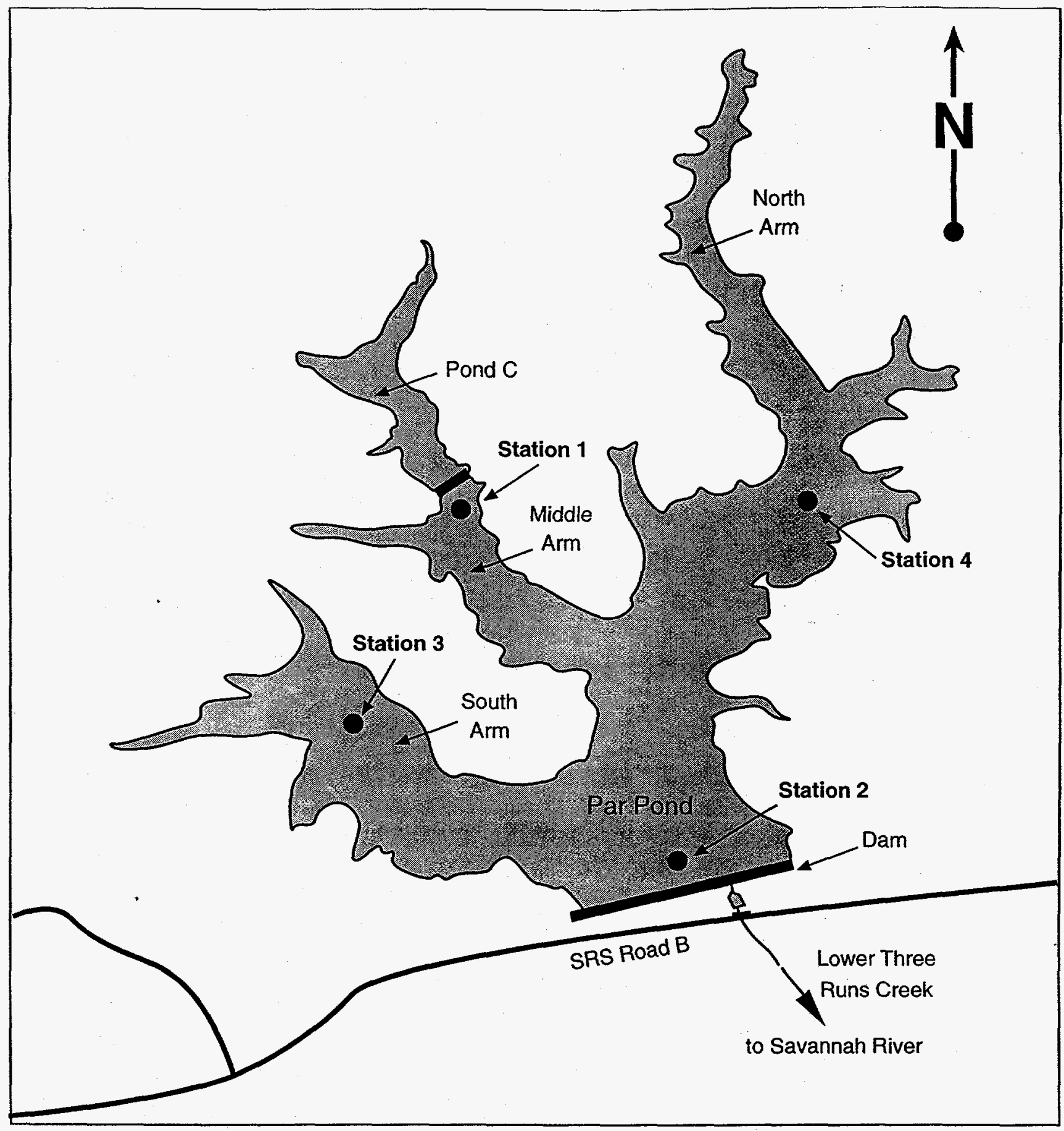

Figure 1. Map of Par Pond indicating water quality sampling locations. February-September 1995.

and Liability Act (CERCLA) unit by the U.S. Environmental Protection Agency. The Par Pond basin below $55 \mathrm{~m}$ (181 feet) above mean sea level remained under water and was not considered part of the CERCLA unit.
Dam repairs were completed in 1994. The refilling of Par Pond to full pool began in August (Figure 2) by discontinuing the siphons that had maintained the water at $55 \mathrm{~m}$ (181 feet) above mean sea level. In October, pumping of water from the 


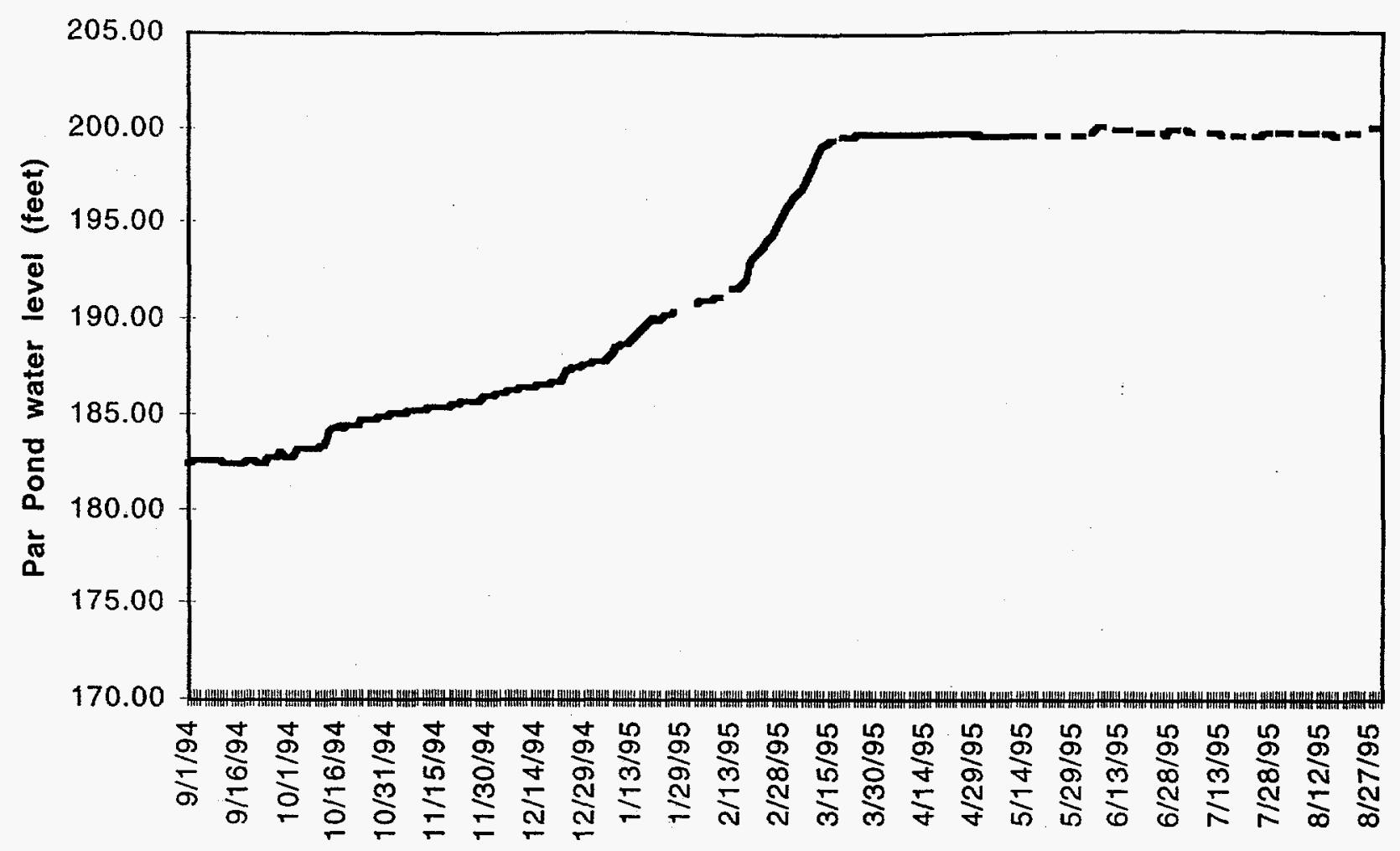

Figure 2. Rate of refill of Par Pond from 55 meters (181 feet) above mean sean level to approximately 61 meters (200 feet) above mean sea level.

Savannah River was initiated to expedite filling of the reservoir to full pool before the spring fishspawning season.

By completing the refill during the cooler months, the likelihood of a fish kill was reduced. The effects of the refill on the reservoir's biological community were of concern. Refill could cause significant nutrient loading as a result of the submersion and subsequent decay of terrestrial vegetation growing on the sediments between 55 and $61 \mathrm{~m}$ (181 and 200 feet) above mean sea level. This nutrient loading could have produced excessive primary productivity in the form of algal growth and subsequent zooplankton growth (zooplankton feed on algae), especially during the warmer months. Zooplankton respiration, the decay of phytoplankton (algae), and the continued decay of submerged terrestrial biomass could have consumed most of the dissolved oxygen in the water, resulting in a major fish kill.
The objective of the sampling activity described in this report was to monitor the water quality conditions in Par Pond during and after the refill by measuring dissolved oxygen, temperature, chlorophyll-a, nitrogen, and phosphorus.

\section{Methods}

Four locations on Par Pond were sampled (Figure 1). These locations matched those of previous studies (Wilde 1985). The Universal Transverse Mercator coordinates are given in Table 1.

Eleven water samples were taken during each sampling event. A total of eight samples was taken from the four locations (one surface and one bottom sample at each location). Two duplicate samples and one quality control sample also were taken. The dissolved oxygen concentration and the water temperature were measured electronically at $1-\mathrm{m}$ intervals 
Table 1. Universal Transverse Mercator coordinates for Par Pond water quality sample locations. February-September 1995.

\begin{tabular}{|c|c|c|}
\hline Sample ID & East coordinate & North coordinate \\
\hline h- & & \\
\hline Station 1 & 449642.976 & 3680714.362 \\
\hline Station 2 & 451591.399 & 3677586.994 \\
\hline Station 3 & 448986.276 & 3678600.502 \\
\hline Station 4 & 452715.582 & 3681542.933 \\
\hline
\end{tabular}

Notes:

Station 1 is located at the dam between Par Pond and Pond C.

Station 2 is located near the dam across Lower Three Runs.

Station 3 is located in the South Arm, near the Savannah River Technology Center Par Pond laboratory and the Par Pond pumphouse.

Station 4 is located in the North Arm.

through the water column at each of the four sample locations.

All in situ water sampling was conducted by personnel of Westinghouse Savannah River Company, Environmental Monitoring Section using established water quality data collection procedures. The Environmental Monitoring Section subcontracted the analyses of nutrients and chlorophyll-a to General Engineering Laboratories, Inc.

Samples were taken approximately twice per month during the reporting period, February through September 1995. Sampling continued through the remainder of 1995 and is scheduled to be completed by the end of September 1996. No samples were filtered. A final report will summarize the results of the monitoring program.

All samples were handled using Standard Operating Procedures as required by the sampling and analysis plan. Collection, preservation, shipping, and chain-of-custody requirements were strictly enforced. Occasionally at the beginning of the study, sample holding times were exceeded. Additionally, several sets of samples were not properly preserved prior to shipment to the analytical laboratory. Factors causing these early lapses were identified and corrected, and the remainder of the samples were collected correctly.

\section{Results}

Dissolved oxygen (DO) is the most critical component of lacustrine ecosystems. A DO concentration of 5 milligrams per liter of water $(\mathrm{mg} / \mathrm{L})$ is considered the minimum capable of supporting most freshwater fishes, although a few species are capable of surviving extended periods in water with less than $2 \mathrm{mg} / \mathrm{L}$ DO (Robison and Buchanan 1988; Pflieger 1975). The DO concentrations, as a function of depth, during this study are given in Appendix A and plotted in Figures 3-6. (Surfer 6.01 for Windows was used to generate all oxygen figures.) March DO concentrations ranged from 5.0 $\mathrm{mg} / \mathrm{L}$ to $10.5 \mathrm{mg} / \mathrm{L}$, while $\mathrm{DO}$ concentrations during the warm months, when the reservoir was stratified (June, July, and August), ranged from less than $1.0 \mathrm{mg} / \mathrm{L}$ to $8.0 \mathrm{mg} / \mathrm{L}$. Previous studies of Par Pond (Chimney et al. 1985) reported DO concentrations from $0.5 \mathrm{mg} / \mathrm{L}$ to $10.1 \mathrm{mg} / \mathrm{L}$. DO concentrations reported from other area lakes include $0.0 \mathrm{mg} / \mathrm{L}$ to $11.3 \mathrm{mg} / \mathrm{L}$ at Lake Thurmond (previously Clarks Hill; Wilde 1985); less than $2.0 \mathrm{mg} / \mathrm{L}$ to $10.0 \mathrm{mg} / \mathrm{L}$ at Lake Murray (McDowell 1977), and less than $0.1 \mathrm{mg} / \mathrm{L}$ to $12.7 \mathrm{mg} / \mathrm{L}$ at $\mathrm{L}$ Lake (Chimney et al. 1989). 


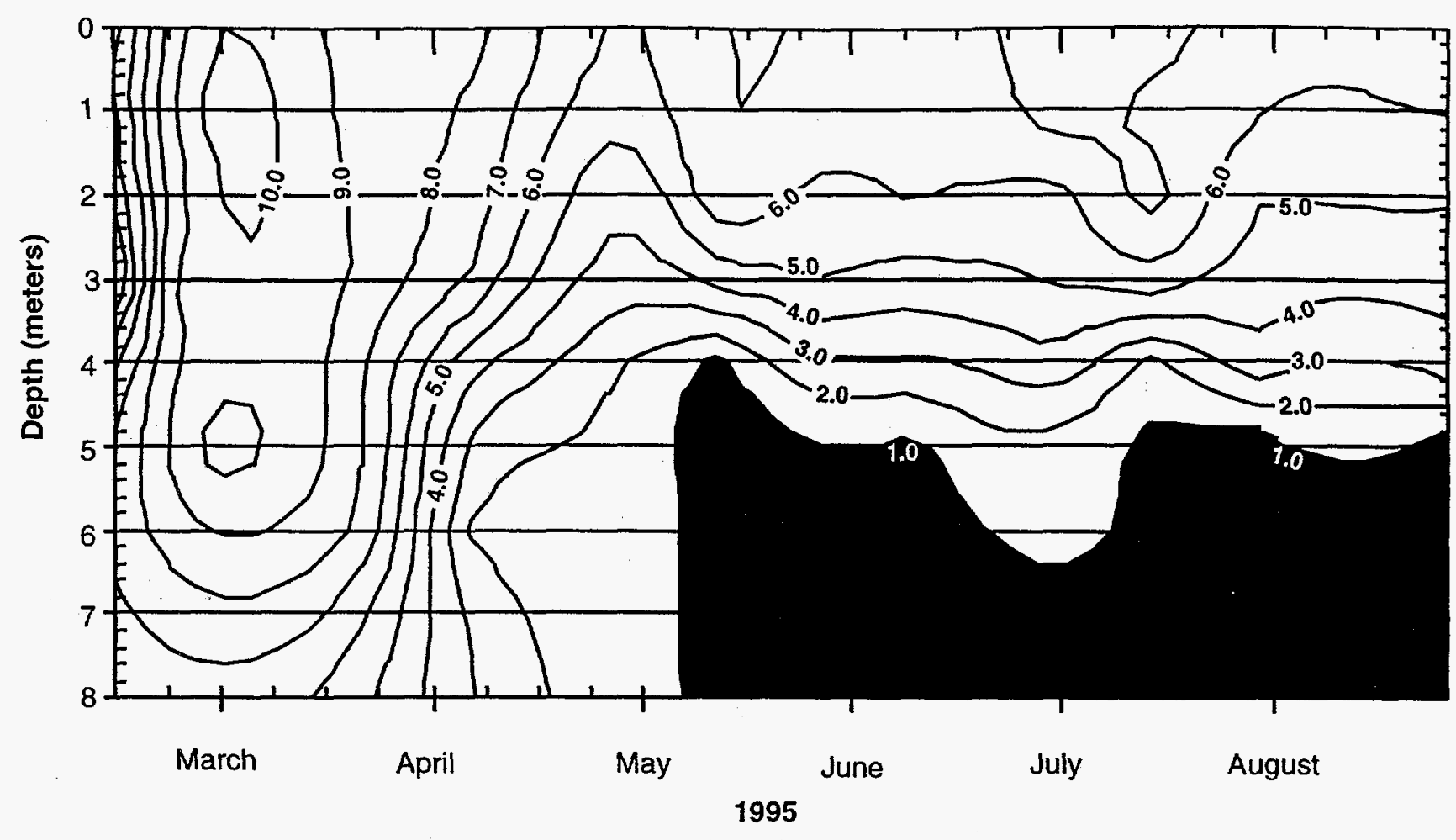

Figure 3. Dissolved oxygen concentrations through the water column at Station 1. February-August 1995.

The development of anoxic conditions below the mixed zone is typical of eutrophic lakes during summer stratification. At Station 2 in June 1985, DO concentrations of less than $1.0 \mathrm{mg} / \mathrm{L}$ were observed at $6 \mathrm{~m}$ and deeper (Wilde 1985); in July $1995 \mathrm{DO}$ at Station 2 fell below $1.0 \mathrm{mg} / \mathrm{L}$ at $4 \mathrm{~m}$ (Figure 4). Station 4 during August was the only station and date where DO of less than $5.0 \mathrm{mg} / \mathrm{L}$ was observed near the surface in both this study (Figure 6) and in 1985 (Chimney et al. 1985). Given the natural variability of DO concentrations due to morphological and climatological factors, the DO results from this study are similar to the 1985 results.

Water temperature determines the solubility of oxygen. The minimum temperature observed was 9.7 degrees Celsius $\left(\left[{ }^{\circ} \mathrm{C}\right] 49\right.$ degrees Fahrenheit $\left.\left[{ }^{\circ} \mathrm{F}\right]\right)$ in February at Station 3 at 2, 3, 4, and $5 \mathrm{~m}$ (Appendix A). The maximum temperature was $33.9^{\circ} \mathrm{C}\left(93^{\circ} \mathrm{F}\right)$ at the surface of Station 4 in June.
Other reported temperature ranges from this area are $7^{\circ} \mathrm{C}$ to $37^{\circ} \mathrm{C}\left(44^{\circ} \mathrm{F}\right.$ to $\left.99^{\circ} \mathrm{F}\right)$ at Par Pond, $6^{\circ} \mathrm{C}$ to $32^{\circ} \mathrm{C}\left(43^{\circ} \mathrm{F}\right.$ to $\left.90^{\circ} \mathrm{F}\right)$ at Lake Thurmond, $1^{\circ} \mathrm{C}$ to $33^{\circ} \mathrm{C}\left(34^{\circ} \mathrm{F}\right.$ to $\left.91^{\circ} \mathrm{F}\right)$ at Monticello Reservoir (Wilde 1985), and $7^{\circ} \mathrm{C}$ to $29^{\circ} \mathrm{C}\left(44^{\circ} \mathrm{F}\right.$ to $\left.84^{\circ} \mathrm{F}\right)$ at Lake Murray (McDowell 1977). Water temperature was not impacted by the refill and the observed temperatures were in the range of expected values.

Chlorophyll-a concentrations are an indirect measure of algal standing crop (biomass) available for zooplankton consumption and a measure of primary productivity in an aquatic ecosystem (Wetzel 1983). Chlorophyll-a concentrations fluctuate seasonally, with the highest concentrations expected in late summer in the top several meters of the water column.

Figure 7 and Appendix B present the chlorophylla concentrations in samples from the top and bot- 


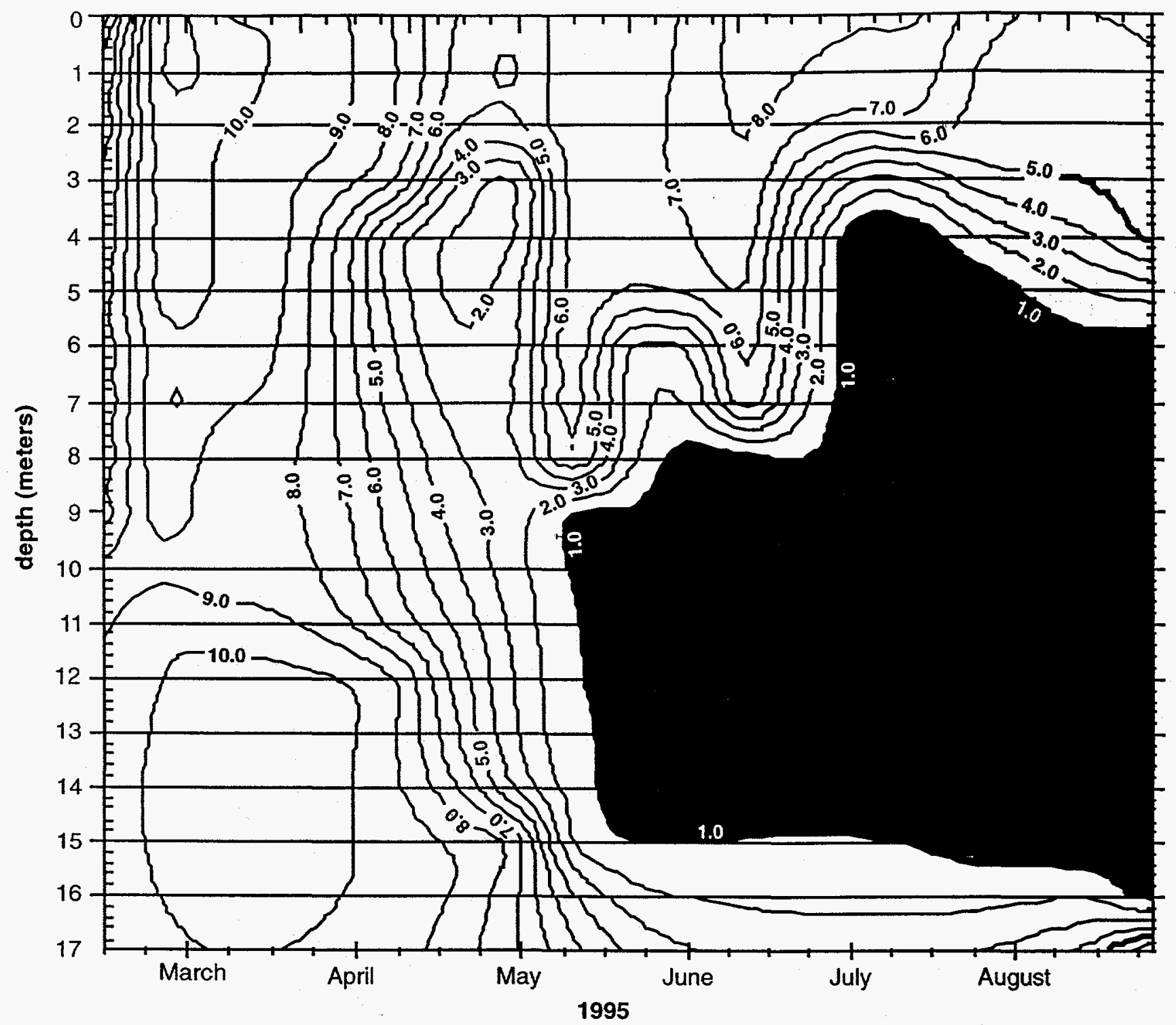

Figure 4. Dissolved oxygen concentrations through the water column at Station 2. February-August 1995.

tom of the four Par Pond sample locations. Chlorophyll-a concentrations ranged from less than 5 milligrams per cubic meter $\left(\mathrm{mg} / \mathrm{m}^{3}\right)$ at all locations through mid-May to $30 \mathrm{mg} / \mathrm{m}^{3}$ during the summer, when phytoplankton productivity is highest. The maximum measured concentration of $60 \mathrm{mg} / \mathrm{m}^{3}$ for the Station 1 bottom sample in June is qualified as an estimated value and considered an outlier. Previous studies reported maximum chlorophyll-a concentrations of $25 \mathrm{mg} / \mathrm{m}^{3}$ in Par Pond (Chimney et al. 1985), $36 \mathrm{mg} / \mathrm{m}^{3}$ at Lake Robinson, and $24.9 \mathrm{mg} / \mathrm{m}^{3}$ at Lake Hartwell (Wilde 1985). An average maximum chlorophyll-a concentration of $21.4 \mathrm{mg} / \mathrm{m}^{3}$ was reported for 13 South Carolina reservoirs (Wilde 1985). With the exception of the one very high value at Station 1 , the observed chlorophyll-a concentrations and seasonal trends in Par Pond are in the range of values and trends expected for southeastern lakes.

Nitrogen is a major plant nutrient and is useful for tracking primary productivity. Nitrogen, available as nitrate, is highest in winter and decreases with increased primary productivity in summer 


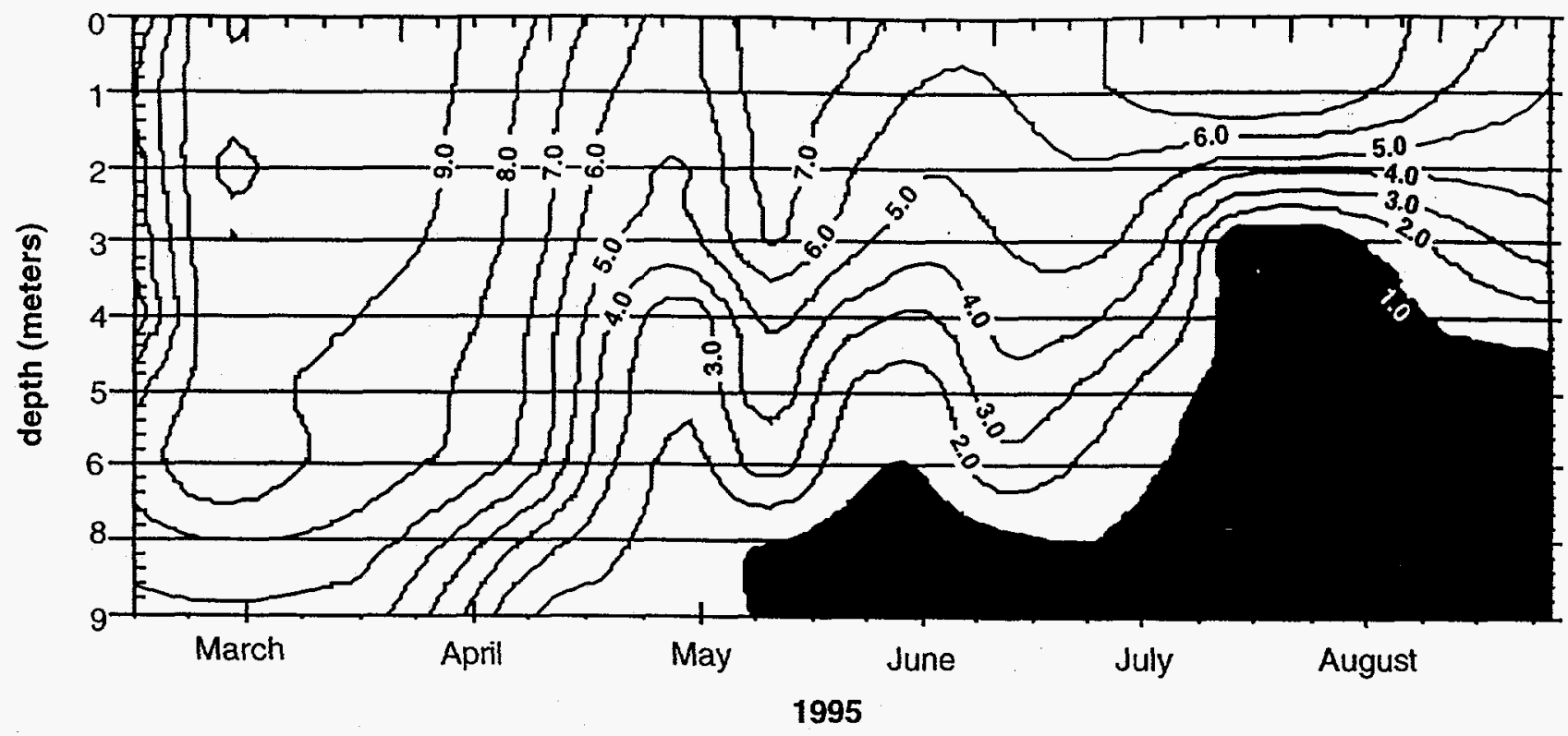

Figure 5. Dissolved oxygen concentrations through the water column at Station 3. February-August 1995.

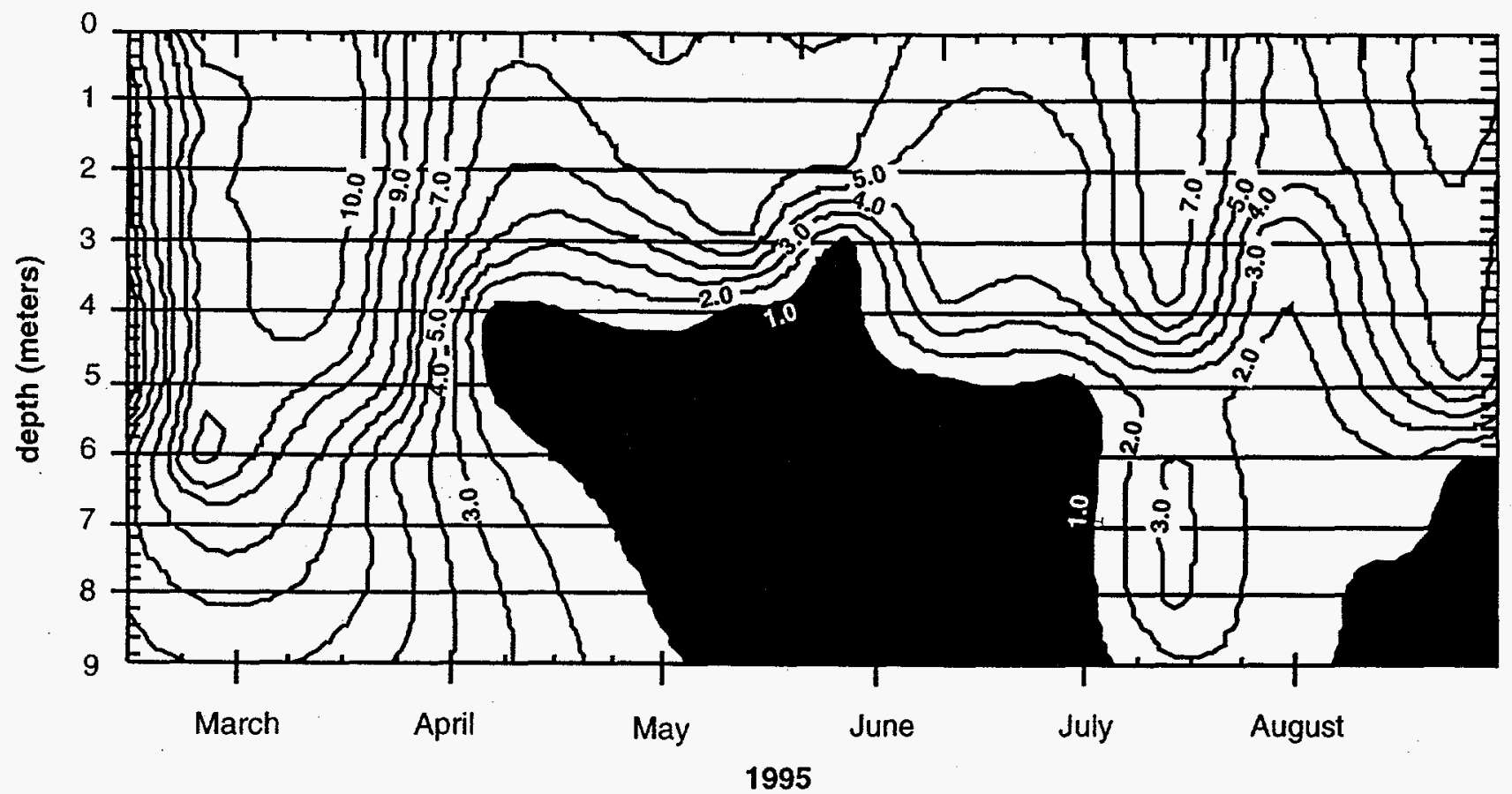

Figure 6. Dissolved oxygen concentrations through the water column at Station 4. February-August 1995.

(Capblancq 1990). Ammonia, a reduced form of nitrogen, accumulates in the hypolimnion as a result of the settling and decay of organic material. Total (Kjeldahl) nitrogen includes ammonia and all forms of organic nitrogen. Nitrogen consumed by productivity or lost to decay is a component of this measurement which represents the conversion of inorganic nitrogen to organic processes.

Nitrogen sampling results are presented in Figures 8-10 and Appendix B. Samples were analyzed for nitrate, ammonia, and total Kjeldahl nitrogen. 

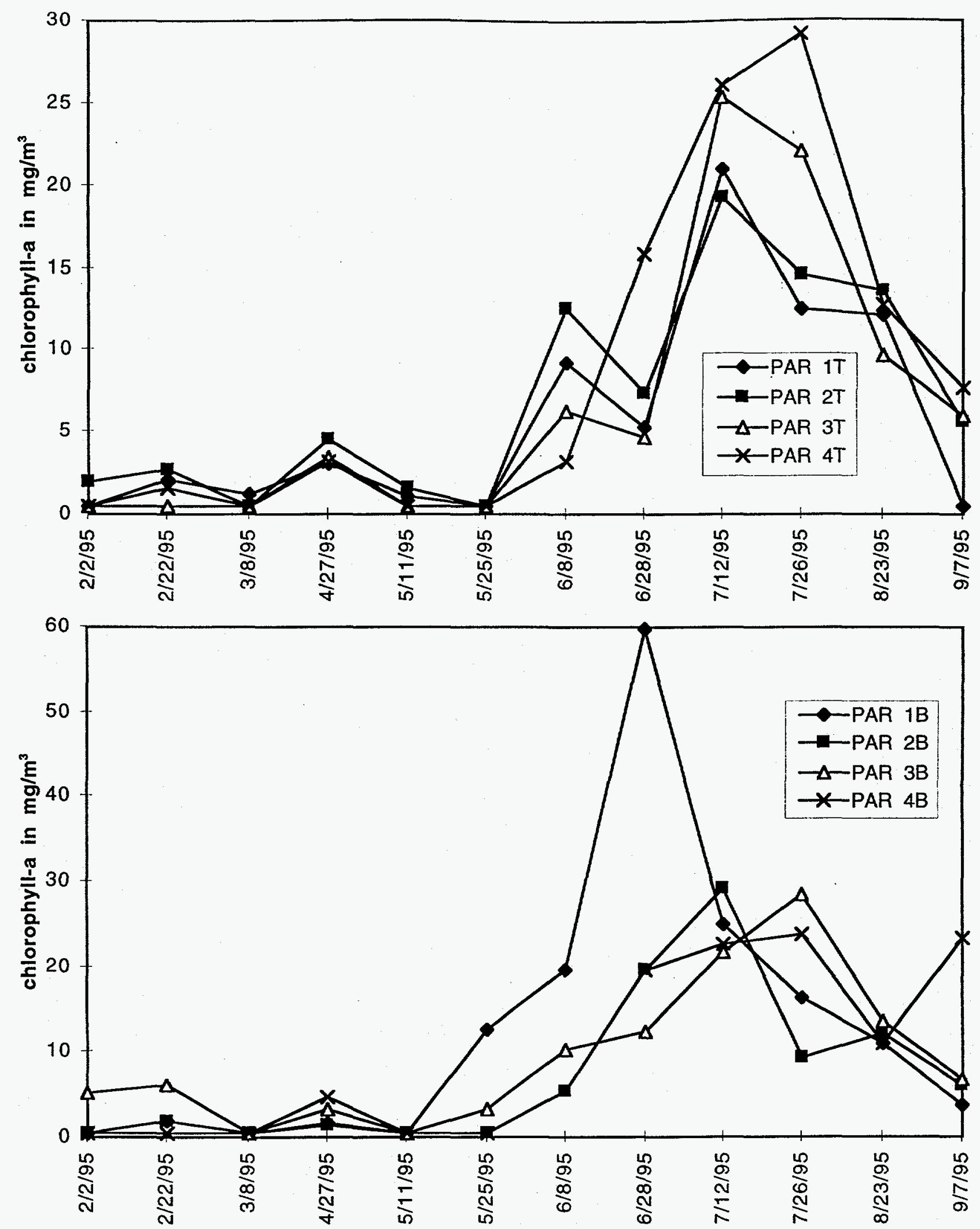

Figure 7. Chlorophyll-a concentrations from surface (upper figure) and bottom (lower figure) water samples at sample locations in Par Pond. Note the difference in scale between the figures. FebruarySeptember 1995. 
Nitrate concentrations ranged from approximately 0 micrograms per liter $(\mu \mathrm{g} / \mathrm{L})$ during the growing season to $180 \mu \mathrm{g} / \mathrm{L}$ in February (Figure 8). Other studies have measured nitrate ranges of $0 \mu \mathrm{g} / \mathrm{L}$ to $660 \mu \mathrm{g} / \mathrm{L}$ in L-Lake, $0 \mu \mathrm{g} / \mathrm{L}$ to $1510 \mu \mathrm{g} / \mathrm{L}$ in Lake Thurmond, $10 \mu \mathrm{g} / \mathrm{L}$ to $210 \mu \mathrm{g} / \mathrm{L}$ in Lake Hartwell, and $7 \mu \mathrm{g} / \mathrm{L}$ to $146 \mu \mathrm{g} / \mathrm{L}$ in Par Pond in 1987 (Chimney et al. 1989). The nitrate values in Par Pond during this study fall within the range expected in southeastern reservoirs.

During the study, ammonia ranged from approximately $30 \mu \mathrm{g} / \mathrm{L}$ to $480 \mu \mathrm{g} / \mathrm{L}$ (Figure 9) and Kjeldahl nitrogen ranged from $50 \mu \mathrm{g} / \mathrm{L}$ to 2040 $\mu \mathrm{g} / \mathrm{L}$ (Figure 10). Ammonia concentrations were previously reported at $0 \mu \mathrm{g} / \mathrm{L}$ to $670 \mu \mathrm{g} / \mathrm{L}$ from Lake Thurmond, $1 \mu \mathrm{g} / \mathrm{L}$ to $240 \mu \mathrm{g} / \mathrm{L}$ from Lake Hartwell, and $2 \mu \mathrm{g} / \mathrm{L}$ to $79 \mu \mathrm{g} / \mathrm{L}$ from Par Pond (Chimney et al. 1989). Ammonia concentrations reported from this study are within the range of expected values.

Total Kjeldahl nitrogen values previously reported were $0 \mu \mathrm{g} / \mathrm{L}$ to $2070 \mu \mathrm{g} / \mathrm{L}$ on L Lake, $0 \mu \mathrm{g} / \mathrm{L}$ to $809 \mu \mathrm{g} / \mathrm{L}$ on Lake Thurmond, and $7 \mu \mathrm{g} / \mathrm{L}$ to 146 $\mu \mathrm{g} / \mathrm{L}$ on Par Pond in 1987 (Chimney et al. 1989). An average low of $22 \mu \mathrm{g} / \mathrm{L}$ and an average high of $690 \mu \mathrm{g} / \mathrm{L}$ was reported for regional lakes (Wilde, 1985). Total Kjeldahl nitrogen at the end of summer (August-September; approximately $1550 \mu \mathrm{g} / \mathrm{L}$ to $2070 \mu \mathrm{g} / \mathrm{L}$ ) was higher than most values reported from other reservoirs but values reported from other months fell within the expected range. The results for all types of nitrogen are indicative of expected seasonal trends.

Phosphorus is considered to be the limiting nutrient in most freshwater aquatic systems (Wetzel 1983). The decay of phytoplankton releases phosphorus as a colloid, which can settle to the bottom or be hydrolyzed to orthophosphate. Orthophosphate is quickly assimilated by the biota and excreted as dissolved phosphorus. Dissolved phosphorus is also released from the substrate by the decay of dead and settled plant material, including phytoplankton (Brown and Barnwell 1987). The values for orthophosphate and total dissolved phosphorus from this study are presented in Figures 11 and 12 and Appendix B.

Orthophosphate values during this study ranged from $11 \mu \mathrm{g} / \mathrm{L}$ to $125 \mu \mathrm{g} / \mathrm{L}$ (Figure 11) and total phosphorus ranged from $30 \mu \mathrm{g} / \mathrm{L}$ to $611 \mu \mathrm{g} / \mathrm{L}$ (Figure 12). Other studies reported orthophosphate values from less than $5 \mu \mathrm{g} / \mathrm{L}$ to $816 \mu \mathrm{g} / \mathrm{L}$ in L Lake (Chimney et al. 1989) and from less than $100 \mu \mathrm{g} / \mathrm{L}$ to $800 \mu \mathrm{g} / \mathrm{L}$ in Lake Thurmond (Wilde 1985). Regional lakes had a range of average orthophosphate values from $2 \mu \mathrm{g} / \mathrm{L}$ to $210 \mu \mathrm{g} / \mathrm{L}$ (Wilde 1985). Total phosphorus values of $14 \mu \mathrm{g} / \mathrm{L}$ to $864 \mu \mathrm{g} / \mathrm{L}$ were reported from L Lake (Chimney et al. 1989) and values of less than $100 \mu \mathrm{g} / \mathrm{L}$ to $520 \mu \mathrm{g} / \mathrm{L}$ were reported from Lake Thurmond (Wilde 1985). Regional lakes had an average total phosphorus range of $7 \mu \mathrm{g} / \mathrm{L}$ to $388 \mu \mathrm{g} / \mathrm{L}$ (Wilde 1985). Phosphorus values reported in this study are similar to those measured in other regional reservoirs. No seasonal trends were detected. Phosphorus concentrations from the May 25, 1995, collection are higher than those from other dates.

\section{Discussion}

Most lentic systems in the Southeast are man-made reservoirs that are eutrophic to some extent. Eutophy is the result of nutrient loading and is characterized by vertical thermal stratification in the warm months. Stratification forms an upper layer (epilimnion) and a lower layer (hypolimnion) separated by a thermocline, which is identified by a large change in temperature over a very short depth. The epilimnion and the hypolimnion are chemically very different. When a reservoir is stratified, the mixing of the water and reaeration, which introduces oxygen into the water column, is restricted to the epilimnion. The epilimnion and the hypolimnion remain distinct; therefore, the hypolimnion becomes anoxic because oxygen used in hypolimnetic biological processes is not replaced with oxygen from the epilimnion. In some cases, 

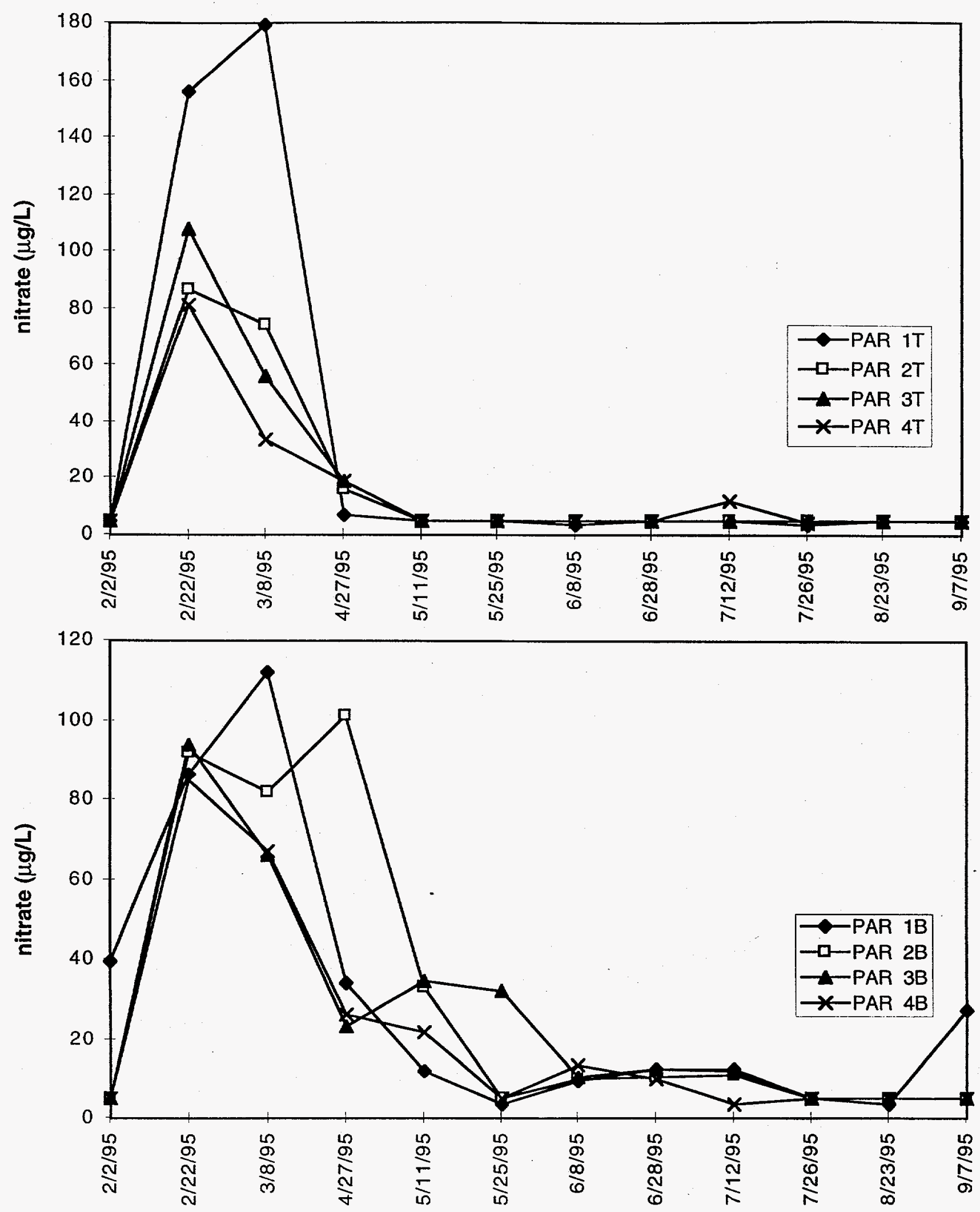

Figure 8. Nitrate concentrations from surface (upper figure) and bottom (lower figure) water samples at sample locations in Par Pond. Note the difference in scale between the figures. February-September 1995. 

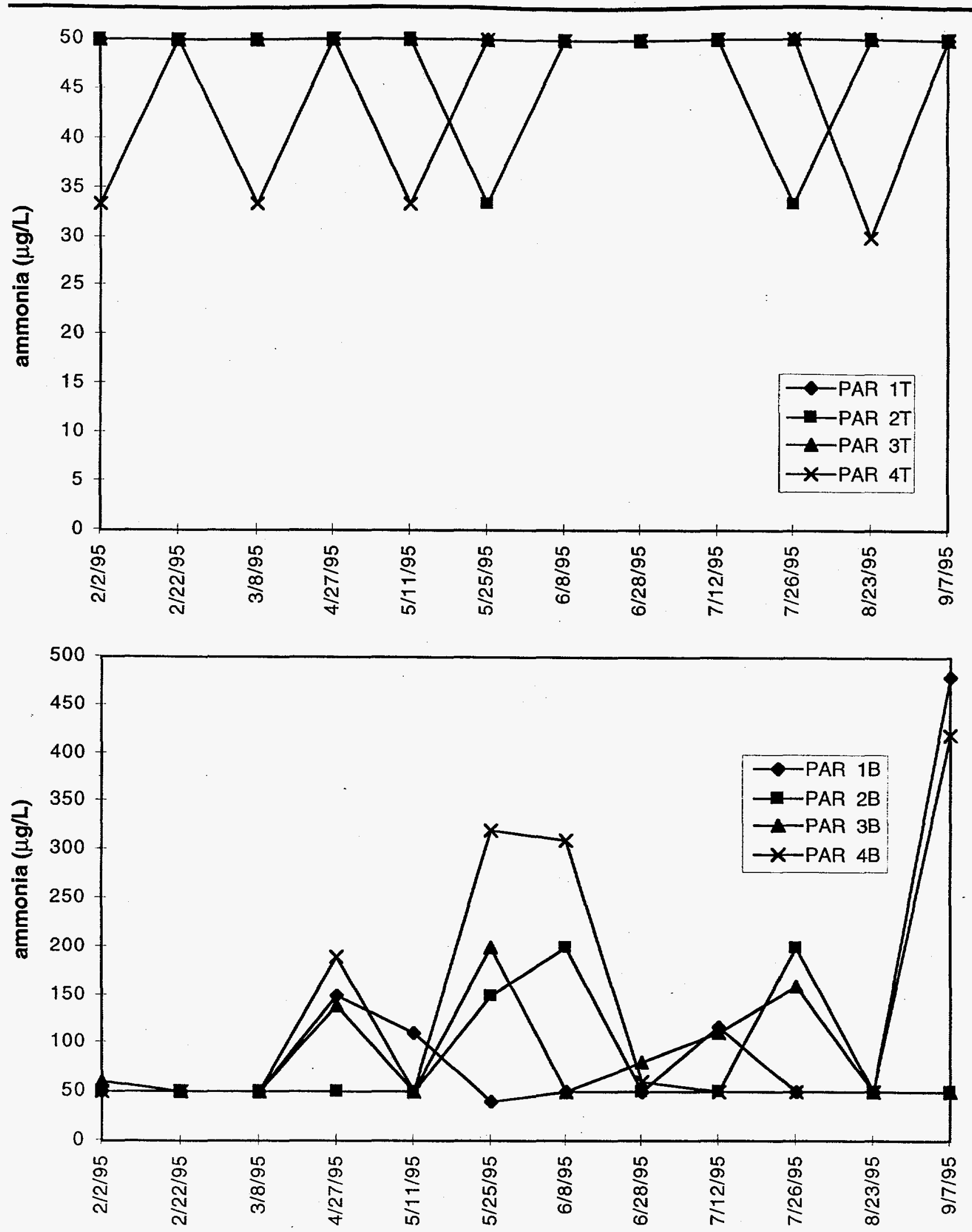

Figure 9. Ammonia concentrations from surface (upper figure) and bottom (lower figure) water samples at sample locations in Par Pond. Note the difference in scale between the figures. February-September 1995. 

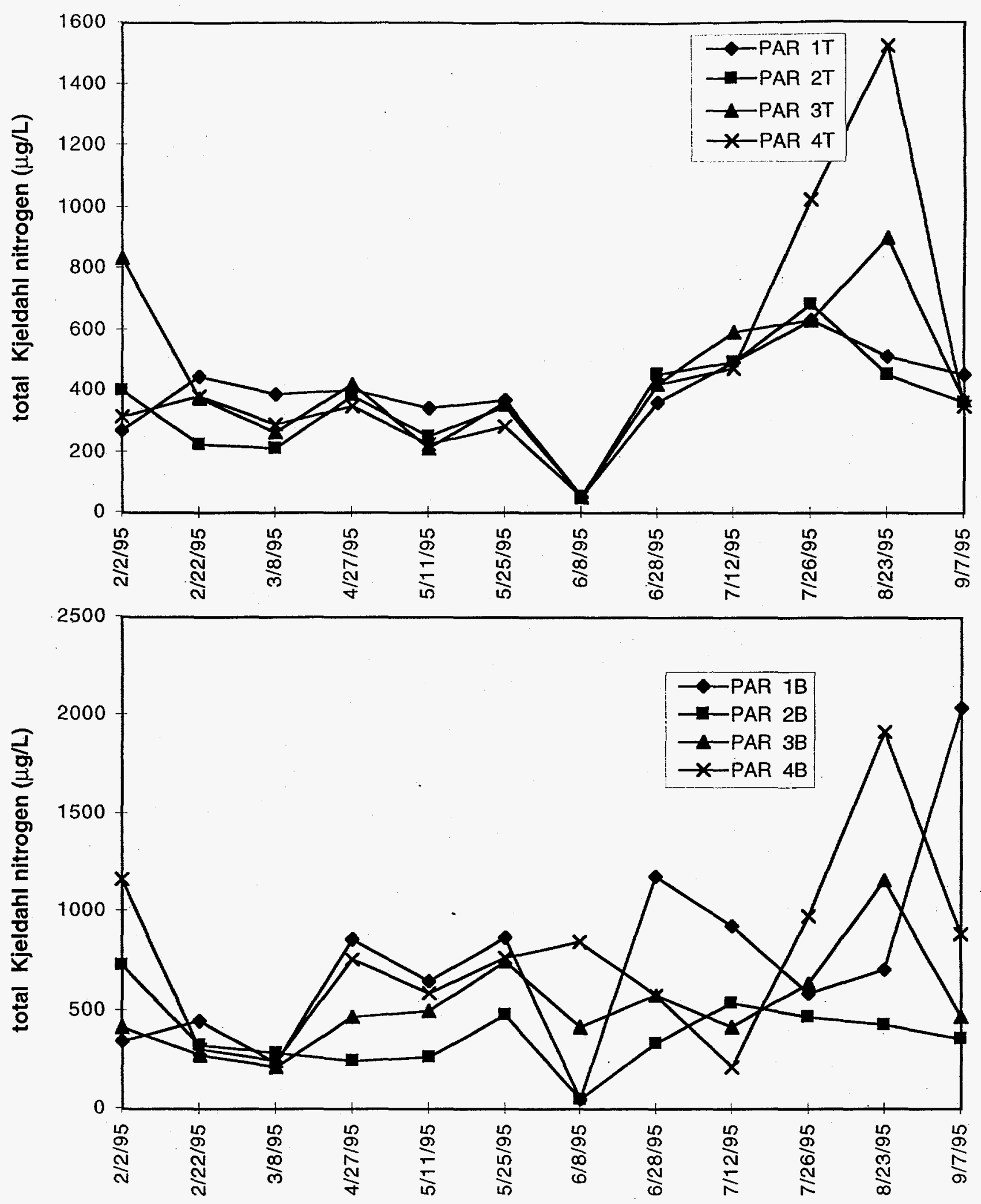

Figure 10. Total Kjeldahl nitrogen concentrations from surface (upper figure) and bottom (lower figure) water samples at sample locations in Par Pond. Note the difference in scale between the figures. February-September 1995. 

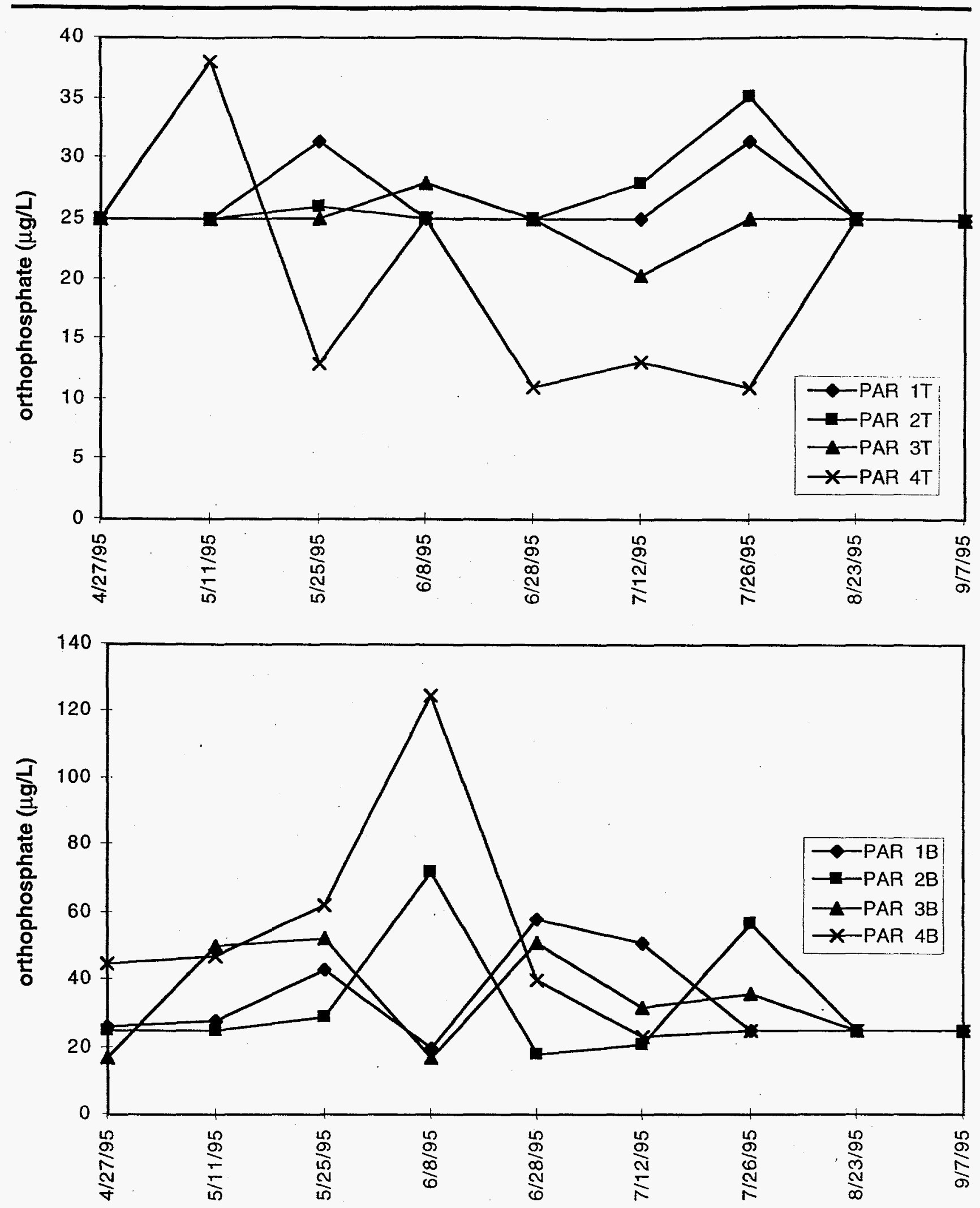

Figure 11. Orthophosphate concentrations from surface (upper figure) and bottom (lower figure) water samples at sample locations in Par Pond. Note the difference in scale between the figures. FebruarySeptember 1995. 

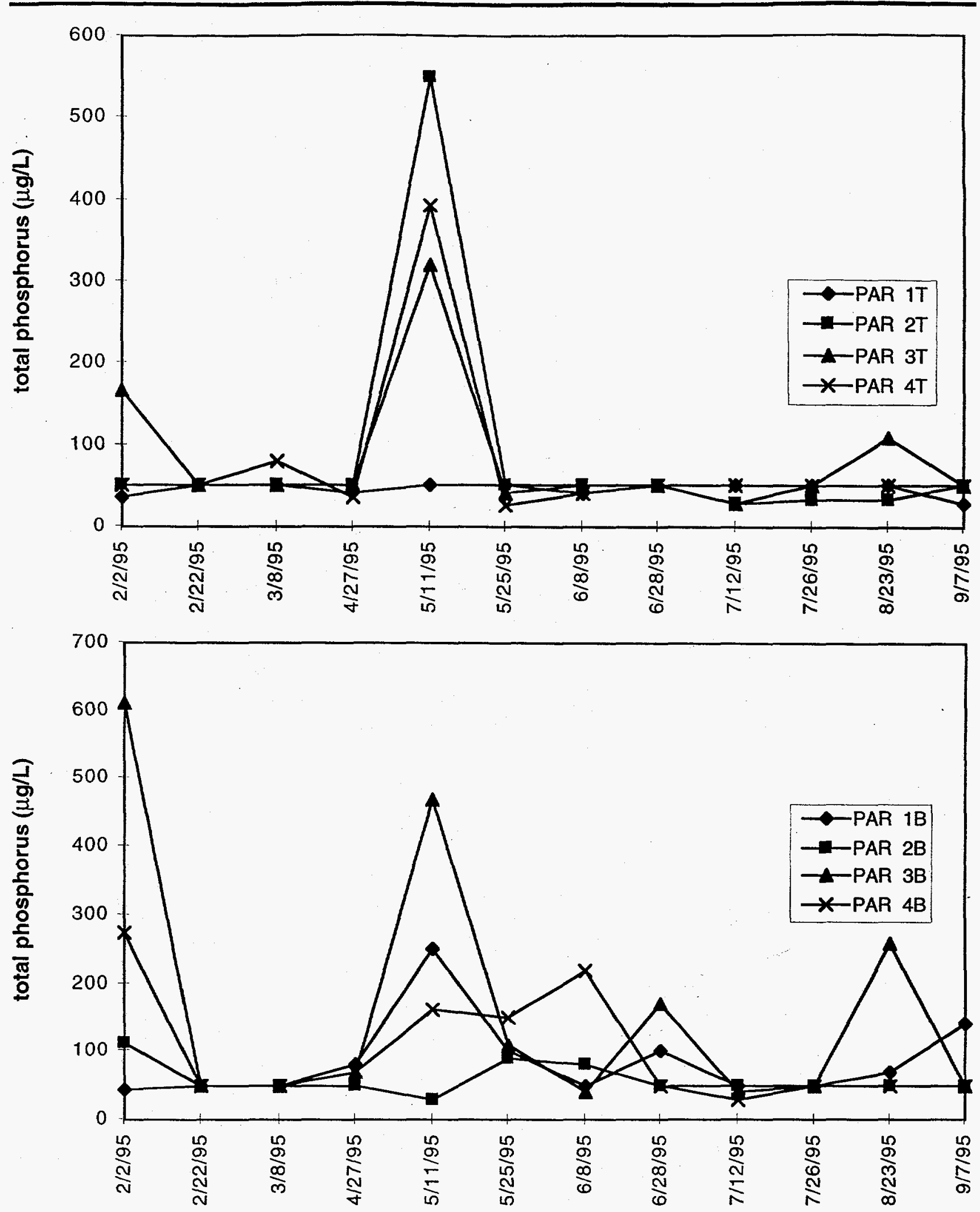

Figure 12. Total dissolved phosphorus concentrations from surface (upper figure) and bottom (lower figure) water samples at sample locations in Par Pond. Note the difference in scale between the figures. February-September 1995. 
high primary productivity in the epilimnion reduces light penetration to lower depths, causing the thermocline to move up in the water column and reducing the depth of the oxygenated epilimnion. This increased productivity in the epilimnion can cause increases in zooplankton biomass and respiration, increasing oxygen demands. In extreme cases, particularly at high water temperatures which decrease oxygen solubility, even the restricted epilimnion becomes depleted of oxygen. If most of the reservoir becomes anoxic, organisms will die.

The development of eutrophic conditions is impacted by meteorology, water currents, terrestrial shading, depth, the input of nutrients, and primary productivity. Of all nutrients, phosphorus is usually the one that limits primary production in reservoirs. Other nutrients, such as nitrogen and carbon, are released by oxygen-consuming decay processes (Brown and Barnwell 1987). When Par Pond was refilled, large amounts of terrestrial vegetation were inundated and began to decay. The release of nutrients from decaying vegetation resulted in the potential for excessive planktonic growth and oxygen depletion; the combination could have resulted in stress and mortality of aquatic organisms, particularly fish.

This study was designed to document anoxia and to provide information regarding its cause, in the event that it caused a fish kill. No fish kill was observed during summer 1995, and dissolved oxygen and nutrient concentrations generally remained within the range expected for regional reservoirs.

When Par Pond was drawn down, its littoral zone was converted from an aquatic to a terrestrial environment. Nutrient cycling in terrestrial systems is very different from that in aquatic systems. Nutrients from senescent plant material migrate down into the soil. During the winter, most plant material that remains on the surface as litter or standing dead biomass is highly refractory, woody material. Because Par Pond was refilled during the winter, much of the nutrients in the terrestrial plant material had migrated into the soil and was unavailable for rapid leaching into the water or was in refractory forms in senescent material and not susceptible to rapid decay by aquatic mechanisms.

Prior to the drawdown, Par Pond had a very rich nutrient store that had accumulated over the years of pumping make-up water from the Savannah River. These nutrients were contained in the phytoplankton, in substantial aquatic macrophyte biomass, and in the thick organic sediments. The drawdown eliminated the most productive macrophyte beds and caused about $65 \%$ of the nutrientrich water in Par Pond to be discharged out of the reservoir. The organic sediment layer in the exposed areas was quickly decomposed, and, with the soil now dry and permeable, nutrients leached deep into the soil profile. The loss of nutrients pumped out of the reservoir during drawdown and the lack of labile nutrients in decaying terrestrial plant material after the refill decreased the likelihood of anoxic conditions caused by decay or nutrient loading.

During the summer, decay of plant material in the hypolimnion decreases because of anoxic conditions. However, much of the terrestrial plant material in Par Pond was located in shallow areas where anoxia was not a consideration and decom: position proceeded unimpeded. The lake destratified during the fall 1995. The continuous decay of terrestrial plant material, coupled with the increased consumption of oxygen by biological processes in the recently oxygenated hypolimnion and the development of new aquatic macrophyte beds, may increase nutrient loading and oxygen demand during the second summer after refill. This study will continue to monitor the Par Pond water chemistry during the second summer after refill to better understand the conditions in the event of a fish kill. 


\section{Summary}

The refill of Par Pond created a potential for the destabilization of the aquatic nutrient balance in the reservoir due to the decay of terrestrial plants that had been growing on the now-inundated, but previously exposed, sediments. It was hoped that the timing of the refill, during the winter when above-ground terrestrial biomass is low and aquatic biotic activity is minimal, along with the refrac- tory nature of winter terrestrial plant material, would prevent the sudden release of nutrients, which could result in eutrophication or anoxia. No fish kill or extreme eutrophic conditions were observed during this study. Par Pond water quality will continue to be monitored for instability during the second summer as the aquatic macrophytes become reestablished and sediment nutrients become more available to the water column. 


\section{References}

Brown, L. C., and T. O. Barnwell, Jr. 1987. The Enhanced Stream Water Quality Models QUAL2E and QUAL2E-UNCAS: Documentation and Users Manual. Environmental Research Laboratory. U.S. Environmental Protection Agency. Athens, GA.

Capblancq, J. 1990. Nutrient dynamics and pelagic food web interactions in oligotrophic and eutrophic environments: An overview. Hydrobiologia 207: $1-14$.

Chimney, M. J., W. R. Cody, and W. M. Starkel. 1985. Final report on the water quality, phytoplankton, and zooplankton of Par Pond and Pond B. January 1984-1985. Vols. I, II, III. - DPST-85-789. Prepared by Environmental \& - Chemical Sciences, Inc. for Savannah River Laboratory. E. I. du Pont de Nemours \& Co. Aiken, SC.

Chimney, M. J., W. M. Starkel, and J. H. Nagle. 1989. L-Lake Water Quality: L-Lake/Steel Creek Biological Monitoring Program. November 1986December 1987. NAI-SR-82. Normandeau Associates Inc. Aiken, SC. DPST-88-651
McDowell, E. B., Jr. 1977. Phosphorus, Planktonic Metabolism and Dissolved Oxygen Depletion in Lake Murray, South Carolina. Master's thesis. School of Public Health, University of South Carolina. Columbia, SC.

Pflieger, W. L. 1975. The Fishes of Missouri. Missouri Department of Conservation. St. Louis, MO.

Robison, H. W. and T. M. Buchanan. 1988. Fishes of Arkansas. University of Arkansas Press. Fayetteville, AK.

Wetzel, R. G. 1983. Limnology. Second Edition. Saunders College Publishing, Philadelphia, PA.

Whicker, F. W., J. E. Pinder II, J. W. Bowling, J. J. Alberts, and I. L. Brisbin, Jr. 1990. Distribution of long-lived radionuclides in an abandoned cooling reservoir. Ecological Monographs 60(4):471-479.

Wilde, E. W. 1985. Compliance of the Savannah River Plant P-Reactor Cooling System with Environmental Regulations. DP-1708. E.I. du Pont de Nemours \& Co. Savannah River Laboratory. Aiken, SC. 
Appendix A

Field Results

Dissolved Oxygen and Temperature

at Four Par Pond Locations

February-September 1995 
Table A-1. Sample location: PAR 1.

\begin{tabular}{|c|c|c|c|c|}
\hline Location & Depth (meters) & Sample date & Temperature $\left({ }^{\circ} \mathrm{C}\right)$ & DO (mg/L) \\
\hline$\because \because$ & -n & nn & n & n \\
\hline PAR 1 & 0 & $2 / 14 / 95$ & 13.5 & 4.70 \\
\hline PAR 1 & 1 & $2 / 14 / 95$ & 13.4 & 4.80 \\
\hline PAR 1 & 2 & $2 / 14 / 95$ & 12.7 & 4.20 \\
\hline PAR 1 & 3 & $2 / 14 / 95$ & 12.1 & 2.40 \\
\hline PAR 1 & 0 & $2 / 21 / 95$ & 13.0 & 8.40 \\
\hline PAR 1 & 1 & $2 / 21 / 95$ & 10.4 & 9.10 \\
\hline PAR 1 & 2 & $2 / 21 / 95$ & 10.2 & 8.50 \\
\hline PAR 1 & 3 & $2 / 21 / 95$ & 10.2 & 9.40 \\
\hline PAR 1 & 4 & $2 / 21 / 95$ & 10.2 & 8.90 \\
\hline PAR 1 & 0 & $3 / 7 / 95$ & 15.2 & 10.00 \\
\hline PAR 1 & 1 & $3 / 7 / 95$ & 14.8 & 10.42 \\
\hline PAR 1 & 2 & $3 / 7 / 95$ & 14.0 & 9.90 \\
\hline PAR 1 & 3 & $3 / 7 / 95$ & 13.4 & 9.71 \\
\hline PAR 1 & 4 & $3 / 7 / 95$ & 13.0 & 9.40 \\
\hline PAR 1 & 5 & $3 / 7 / 95$ & 13.0 & 10.53 \\
\hline PAR 1 & 0 & $3 / 21 / 95$ & 15.8 & 8.30 \\
\hline PAR 1 & 1 & $3 / 21 / 95$ & 15.4 & 8.30 \\
\hline PAR 1 & 2 & $3 / 21 / 95$ & 15.2 & 8.60 \\
\hline PAR 1 & 3 & $3 / 21 / 95$ & 14.6 & 8.70 \\
\hline PAR 1 & 4 & $3 / 21 / 95$ & 13.9 & 7.90 \\
\hline PAR 1 & 5 & $3 / 21 / 95$ & 13.0 & 7.90 \\
\hline PAR 1 & 6 & $3 / 21 / 95$ & 13.0 & 7.60 \\
\hline PAR 1 & 0 & $4 / 4 / 95$ & 18.7 & 8.40 \\
\hline PAR 1 & 1 & $4 / 4 / 95$ & 18.4 & 7.90 \\
\hline PAR 1 & 2 & $4 / 4 / 95$ & 18.0 & 7.60 \\
\hline PAR 1 & 3 & $4 / 4 / 95$ & 17.3 & 7.10 \\
\hline PAR 1 & 4 & $4 / 4 / 95$ & 16.7 & 4.40 \\
\hline PAR 1 & 5 & $4 / 4 / 95$ & 15.6 & 2.80 \\
\hline PAR 1 & 6 & $4 / 4 / 95$ & 15.3 & 1.90 \\
\hline PAR 1 & 0 & $4 / 26 / 95$ & 22.5 & 5.80 \\
\hline PAR 1 & 1 & $4 / 26 / 95$ & 22.2 & 5.40 \\
\hline PAR 1 & 2 & $4 / 26 / 95$ & 22.0 & 3.90 \\
\hline PAR 1 & 3 & $4 / 26 / 95$ & 21.2 & 3.60 \\
\hline PAR 1 & 4 & $4 / 26 / 95$ & 20.3 & 2.00 \\
\hline PAR 1 & 5 & $4 / 26 / 95$ & 19.0 & 1.80 \\
\hline PAR 1 & 6 & $4 / 26 / 95$ & 16.9 & 1.70 \\
\hline PAR 1 & 0 & $5 / 10 / 95$ & 25.0 & 7.00 \\
\hline PAR 1 & 1 & $5 / 10 / 95$ & 25.0 & 7.00 \\
\hline PAR 1 & 2 & $5 / 10 / 95$ & 24.8 & 6.80 \\
\hline PAR 1 & 3 & $5 / 10 / 95$ & 22.9 & 4.50 \\
\hline PAR 1 & 4 & $5 / 10 / 95$ & 21.4 & 0.70 \\
\hline PAR 1 & 5 & $5 / 10 / 95$ & 19.7 & 0.50 \\
\hline PAR 1 & 6 & $5 / 10 / 95$ & 18.5 & 0.60 \\
\hline PAR 1 & 0 & $5 / 24 / 95$ & 29.3 & 6.90 \\
\hline PAR 1 & 1 & $5 / 24 / 95$ & 28.1 & 6.60 \\
\hline
\end{tabular}


Table A-1 (continued). Sample location: PAR 1.

Location

PAR

PAR 1

PAR 1

PAR 1

PAR 1

PAR 1

PAR 1

PAR 1

PAR 1

PAR 1

PAR 1

PAR 1

PAR 1

PAR 1

PAR 1

PAR 1

PAR 1

PAR 1

PAR 1

PAR 1

PAR 1

PAR 1

PAR 1

PAR 1

PAR 1

PAR 1

PAR 1

PAR 1

PAR 1

RAR 1

PAR 1

PAR 1

PAR 1

PAR 1

PAR 1

PAR 1

PAR 1

PAR 1

PAR 1

PAR 1

PAR 1

PAR 1

PAR 1

PAR 1

PAR 1
Depth (meters)

Sample date

Temperature $\left({ }^{\circ} \mathrm{C}\right)$

DO $(\mathrm{mg} / \mathrm{L})$

\begin{tabular}{ll}
\hline $5 / 24 / 95$ & 26.4 \\
\hline $5 / 24 / 95$ & 25.7 \\
\hline $5 / 24 / 95$ & 24.9 \\
\hline $5 / 24 / 95$ & 24.5 \\
\hline $6 / 7 / 95$ & 27.2 \\
\hline $6 / 7 / 95$ & 27.1 \\
$6 / 7 / 95$ & 26.6 \\
$6 / 7 / 95$ & 25.6 \\
\hline $6 / 7 / 95$ & 24.8 \\
$6 / 7 / 95$ & 22.9 \\
\hline $6 / 7 / 95$ & 21.0 \\
\hline $6 / 27 / 95$ & 30.3 \\
\hline $6 / 27 / 95$ & 28.7 \\
\hline $6 / 27 / 95$ & 28.4 \\
$6 / 27 / 95$ & 26.6 \\
\hline $6 / 27 / 95$ & 25.1
\end{tabular}

26.4

24.9

27.2

27.1

26.6

25.6

22.9

21.0

0

1

3

4

5

$6 / 27 / 95$

$6 / 27 / 95$

$6 / 27 / 95$

$6 / 27 / 95$

$6 / 27 / 95$

$7 / 11 / 95$

$7 / 11 / 95$

$7 / 11 / 95$

$7 / 11 / 95$

$7 / 11 / 95$

$7 / 11 / 95$

$7 / 11 / 95$

$7 / 25 / 95$

$7 / 25 / 95$

$7 / 25 / 95$

$7 / 25 / 95$

$7 / 25 / 95$

$7 / 25 / 95$

$7 / 25 / 95$

$7 / 25 / 95$

7/25/95

$8 / 22 / 95$

$8 / 22 / 95$

$8 / 22 / 95$

$8 / 22 / 95$

$8 / 22 / 95$

$8 / 22 / 95$

$8 / 22 / 95$

$8 / 22 / 95$

$8 / 22 / 95$
25.1

24.7

24.0

23.5

21.4

31.4

31.3

30.3

30.0

28.7

25.8

22.2

30.5

29.9

29.6

29.5

29.3

25.9

23.6

20.7

21.3

30.1

29.7

29.4

29.2

28.9

25.7

22.4

21.2

21.0
5.70

5.10

2.91

0.89

6.70

6.30

6.10

4.60

2.90

0.70

0.80

7.06

7.47

5.59

5.18

3.68

1.68

1.22

0.77

0.65

7.48

6.70

7.41

5.70

1.69

0.71

0.95

6.33

6.14

5.07

4.44

3.79

0.32

0.34

0.43

0.53

6.42

6.09

5.11

4.31

3.62

0.39

0.42

0.49

0.54 
Table A-1 (continued). Sample location: PAR 1.

\begin{tabular}{|c|c|c|c|c|}
\hline Location & Depth (meters) & Sample date & Temperature $\left({ }^{\circ} \mathrm{C}\right)$ & $\mathrm{DO}(\mathrm{mg} / \mathrm{L})$ \\
\hline $0 \times 2 \times 2$ & a, & & й & \\
\hline PAR 1 & 0 & $9 / 7 / 95$ & 29.3 & 7.00 \\
\hline PAR 1 & 1 & $9 / 7 / 95$ & 28.7 & 6.33 \\
\hline PAR 1 & 2 & $9 / 7 / 95$ & 26.8 & 5.22 \\
\hline PAR 1 & 3 & $9 / 7 / 95$ & 26.1 & 4.40 \\
\hline PAR 1 & 4 & $9 / 7 / 95$ & 26.1 & 3.93 \\
\hline PAR 1 & 5 & $9 / 7 / 95$ & 25.9 & 1.30 \\
\hline PAR 1 & 6 & $9 / 7 / 95$ & 23.3 & 0.97 \\
\hline PAR 1 & 7 & $9 / 7 / 95$ & 21.2 & 0.71 \\
\hline PAR 1 & 8 & $9 / 7 / 95$ & 20.5 & 0.40 \\
\hline PAR 1 & 0 & $9 / 27 / 95$ & 24.2 & 6.54 \\
\hline PAR 1 & 1 & $9 / 27 / 95$ & 24.2 & 6.38 \\
\hline PAR 1 & 2 & $9 / 27 / 95$ & 24.0 & 4.75 \\
\hline PAR 1 & 3 & $9 / 27 / 95$ & 23.6 & 2.26 \\
\hline PAR 1 & 4 & $9 / 27 / 95$ & 23.2 & 1.81 \\
\hline
\end{tabular}


Table A-2. Sample location: PAR 2.

Location

Depth (meters)

Sample date Temperature ( $\left.{ }^{\circ} \mathrm{C}\right)$

DO (mg/L)

\begin{tabular}{|c|c|c|c|c|}
\hline PAR 2 & 0 & $2 / 14 / 95$ & 13.2 & 3.10 \\
\hline PAR 2 & 1 & $2 / 14 / 95$ & 13.3 & 4.70 \\
\hline PAR 2 & 2 & $2 / 14 / 95$ & 13.5 & 4.90 \\
\hline PAR 2 & 3 & $2 / 14 / 95$ & 13.2 & 5.70 \\
\hline PAR 2 & 4 & $2 / 14 / 95$ & 13.4 & 5.80 \\
\hline PAR 2 & 5 & $2 / 14 / 95$ & 13.2 & 6.30 \\
\hline PAR 2 & 6 & $2 / 14 / 95$ & 13.1 & 6.20 \\
\hline PAR 2 & 7 & $2 / 14 / 95$ & 13.0 & 6.00 \\
\hline PAR 2 & 8 & $2 / 14 / 95$ & 13.5 & 6.20 \\
\hline PAR 2 & 9 & $2 / 14 / 95$ & 13.7 & 6.50 \\
\hline PAR 2 & 0 & $2 / 21 / 95$ & 12.2 & 10.80 \\
\hline PAR 2 & 1 & $2 / 21 / 95$ & 10.5 & 10.70 \\
\hline PAR 2 & 2 & $2 / 21 / 95$ & 10.4 & 10.60 \\
\hline PAR 2 & 3 & $2 / 21 / 95$ & 10.8 & 10.50 \\
\hline PAR 2 & 4 & $2 / 21 / 95$ & 10.5 & 9.70 \\
\hline PAR 2 & 5 & $2 / 21 / 95$ & 10.4 & 10.10 \\
\hline PAR 2 & 6 & $2 / 21 / 95$ & 10.4 & 10.50 \\
\hline PAR 2 & 7 & $2 / 21 / 95$ & 10.4 & 10.40 \\
\hline PAR 2 & 8 & $2 / 21 / 95$ & 10.4 & 9.40 \\
\hline PAR 2 & 9 & $2 / 21 / 95$ & 10.3 & 10.30 \\
\hline PAR 2 & 10 & $2 / 21 / 95$ & 10.6 & 9.90 \\
\hline PAR 2 & 0 & $3 / 7 / 95$ & 12.7 & 10.80 \\
\hline PAR 2 & 1 & $3 / 7 / 95$ & 12.5 & 11.40 \\
\hline PAR 2 & 2 & $3 / 7 / 95$ & 12.2 & 10.90 \\
\hline PAR 2 & 3 & $3 / 7 / 95$ & 12.0 & 9.90 \\
\hline PAR 2 & 4 & $3 / 7 / 95$ & 11.7 & 9.80 \\
\hline PAR 2 & 5 & $3 / 7 / 95$ & 11.6 & 9.90 \\
\hline PAR 2 & 6 & $3 / 7 / 95$ & 11.4 & 9.90 \\
\hline PAR 2 & 7 & $3 / 7 / 95$ & 11.1 & 9.40 \\
\hline PAR 2 & 8 & $3 / 7 / 95$ & 11.0 & 9.50 \\
\hline PAR 2 & 9 & $3 / 7 / 95$ & 10.8 & 8.40 \\
\hline PAR 2 & 10 & $3 / 7 / 95$ & 10.6 & 8.10 \\
\hline PAR 2 & 11 & $3 / 7 / 95$ & 10.5 & 7.80 \\
\hline PAR 2 & 0 & $3 / 21 / 95$ & 15.8 & 9.90 \\
\hline PAR 2 & 1 & $3 / 21 / 95$ & 15.6 & 9.80 \\
\hline PAR 2 & 2 & $3 / 21 / 95$ & 15.5 & 9.70 \\
\hline PAR 2 & 3 & $3 / 21 / 95$ & 15.0 & 9.50 \\
\hline PAR 2 & 4 & $3 / 21 / 95$ & 14.9 & 9.30 \\
\hline PAR 2 & 5 & $3 / 21 / 95$ & 14.4 & 8.80 \\
\hline PAR 2 & 6 & $3 / 21 / 95$ & 14.2 & 8.70 \\
\hline PAR 2 & 7 & $3 / 21 / 95$ & 14.0 & 8.50 \\
\hline PAR 2 & 8 & $3 / 21 / 95$ & 13.5 & 8.40 \\
\hline PAR 2 & 9 & $3 / 21 / 95$ & 13.4 & 8.20 \\
\hline PAR 2 & 10 & $3 / 21 / 95$ & 13.1 & 7.90 \\
\hline PAR 2 & 11 & $3 / 21 / 95$ & 13.0 & 8.10 \\
\hline
\end{tabular}


Table A-2 (continued). Sample location: PAR 2.

\begin{tabular}{|c|c|c|c|c|}
\hline Location & Depth (meters) & Sample date & Temperature $\left({ }^{\circ} \mathrm{C}\right)$ & DO (mg/L) \\
\hline मी & mal & man & andm & ma \\
\hline PAR 2 & 12 & $3 / 21 / 95$ & 12.8 & 8.30 \\
\hline PAR 2 & 0 & $4 / 4 / 95$ & 18.0 & 8.80 \\
\hline PAR 2 & 1 & $4 / 4 / 95$ & 17.9 & 8.60 \\
\hline PAR 2 & 2 & $4 / 4 / 95$ & 17.8 & 8.30 \\
\hline PAR 2 & 3 & $4 / 4 / 95$ & 17.5 & 8.50 \\
\hline PAR 2 & 4 & $4 / 4 / 95$ & $\overline{17.3}$ & 8.50 \\
\hline PAR 2 & 5 & $4 / 4 / 95$ & 17.3 & 8.00 \\
\hline PAR 2 & 6 & $4 / 4 / 95$ & 15.1 & 3.70 \\
\hline PAR 2 & 7 & $4 / 4 / 95$ & 14.6 & 4.10 \\
\hline PAR 2 & 8 & $4 / 4 / 95$ & 13.8 & 4.50 \\
\hline PAR 2 & 9 & $4 / 4 / 95$ & 13.4 & 5.00 \\
\hline PAR 2 & 10 & $4 / 4 / 95$ & 13.0 & 5.60 \\
\hline PAR 2 & 11 & $4 / 4 / 95$ & 13.1 & 5.90 \\
\hline PAR 2 & 12 & $4 / 4 / 95$ & 12.7 & 6.40 \\
\hline PAR 2 & 0 & $4 / 26 / 95$ & 21.7 & 6.80 \\
\hline PAR 2 & 1 & $4 / 26 / 95$ & 21.0 & 4.80 \\
\hline PAR 2 & 2 & $4 / 26 / 95$ & 20.8 & 4.70 \\
\hline PAR 2 & 3 & $4 / 26 / 95$ & 20.6 & 4.70 \\
\hline PAR 2 & 4 & $4 / 26 / 95$ & 20.3 & 1.20 \\
\hline PAR 2 & 5 & $4 / 26 / 95$ & 16.9 & 1.30 \\
\hline PAR 2 & 6 & $4 / 26 / 95$ & 15.2 & 2.00 \\
\hline PAR 2 & 7 & $4 / 26 / 95$ & 14.6 & 2.10 \\
\hline PAR 2 & 8 & $4 / 26 / 95$ & 14.5 & 2.40 \\
\hline PAR 2 & $\overline{9}$ & $4 / 26 / 95$ & 14.3 & 2.40 \\
\hline PAR 2 & 10 & $4 / 26 / 95$ & 14.2 & 2.60 \\
\hline PAR 2 & 11 & $4 / 26 / 95$ & 13.9 & 2.70 \\
\hline PAR 2 & 12 & $4 / 26 / 95$ & 13.9 & 3.10 \\
\hline PAR 2 & 13 & $4 / 26 / 95$ & 13.7 & 3.40 \\
\hline PAR 2 & 14 & $4 / 26 / 95$ & 13.6 & 3.50 \\
\hline PAR 2 & 15 & $4 / 26 / 95$ & 13.8 & 4.30 \\
\hline PAR 2 & $\overline{0}$ & $5 / 10 / 95$ & 23.1 & 7.00 \\
\hline PAR 2 & 1 & $5 / 10 / 95$ & 23.1 & 6.90 \\
\hline PAR 2 & 2 & $5 / 10 / 95$ & 23.1 & 7.00 \\
\hline PAR 2 & 3 & $5 / 10 / 95$ & 23.1 & 6.90 \\
\hline PAR 2 & 4 & $5 / 10 / 95$ & 23.0 & 6.80 \\
\hline PAR 2 & 5 & $5 / 10 / 95$ & 23.1 & 6.90 \\
\hline PAR 2 & 6 & $5 / 10 / 95$ & 23.1 & 7.00 \\
\hline PAR 2 & 7 & $5 / 10 / 95$ & 23.0 & 6.70 \\
\hline PAR 2 & 8 & $5 / 10 / 95$ & 22.9 & 6.30 \\
\hline PAR 2 & 9 & $5 / 10 / 95$ & 20.1 & 0.60 \\
\hline PAR 2 & 10 & $5 / 10 / 95$ & 17.7 & 0.80 \\
\hline PAR 2 & 11 & $5 / 10 / 95$ & 15.0 & 0.90 \\
\hline PAR 2 & 12 & $5 / 10 / 95$ & 14.7 & 1.00 \\
\hline PAR 2 & 13 & $5 / 10 / 95$ & 14.6 & 1.10 \\
\hline PAR 2 & 14 & $5 / 10 / 95$ & 14.5 & 1.20 \\
\hline
\end{tabular}


Table A-2 (continued). Sample location: PAR 2.

Location

Depth (meters)

Sample date

Temperature $\left({ }^{\circ} \mathrm{C}\right)$

\begin{tabular}{|c|c|c|c|c|}
\hline PAR 2 & 15 & $5 / 10 / 95$ & 14.4 & 1.30 \\
\hline PAR 2 & 0 & $5 / 24 / 95$ & 26.6 & 6.30 \\
\hline PAR 2 & 1 & $5 / 24 / 95$ & 25.5 & 6.50 \\
\hline PAR 2 & 2 & $5 / 24 / 95$ & 25.5 & 6.50 \\
\hline PAR 2 & 3 & $5 / 24 / 95$ & 25.5 & 6.40 \\
\hline PAR 2 & 4 & $5 / 24 / 95$ & 25.2 & 5.90 \\
\hline PAR 2 & 5 & $5 / 24 / 95$ & 24.6 & 5.80 \\
\hline PAR 2 & 6 & $5 / 24 / 95$ & 20.9 & 0.40 \\
\hline PAR 2 & 7 & $5 / 24 / 95$ & 17.6 & 0.45 \\
\hline PAR 2 & 8 & $5 / 24 / 95$ & 15.9 & 0.54 \\
\hline PAR 2 & 9 & $5 / 24 / 95$ & 15.3 & 0.58 \\
\hline PAR 2 & 10 & $5 / 24 / 95$ & 15.1 & 0.61 \\
\hline PAR 2 & 11 & $5 / 24 / 95$ & 14.8 & 0.64 \\
\hline PAR 2 & 12 & $5 / 24 / 95$ & 14.5 & 0.75 \\
\hline PAR 2 & 13 & $5 / 24 / 95$ & 14.3 & 0.82 \\
\hline PAR 2 & 0 & $6 / 7 / 95$ & 26.9 & 8.40 \\
\hline PAR 2 & 1 & $6 / 7 / 95$ & 26.9 & 8.40 \\
\hline PAR 2 & 2 & $6 / 7 / 95$ & 26.8 & 8.20 \\
\hline PAR 2 & 3 & $6 / 7 / 95$ & 26.8 & 8.10 \\
\hline PAR 2 & 4 & $6 / 7 / 95$ & 26.8 & 8.00 \\
\hline PAR 2 & 5 & $6 / 7 / 95$ & 26.7 & 7.40 \\
\hline PAR 2 & 6 & $6 / 7 / 95$ & 26.7 & 6.60 \\
\hline PAR 2 & 7 & $6 / 7 / 95$ & 26.6 & 6.10 \\
\hline PAR 2 & 8 & $6 / 7 / 95$ & 23.5 & 0.40 \\
\hline PAR 2 & 9 & $6 / 7 / 95$ & 19.4 & 0.48 \\
\hline PAR 2 & 10 & $6 / 7 / 95$ & 16.9 & 0.60 \\
\hline PAR 2 & 11 & $6 / 7 / 95$ & 16.0 & 0.60 \\
\hline PAR 2 & 12 & $6 / 7 / 95$ & 15.6 & 0.57 \\
\hline PAR 2 & 13 & $6 / 7 / 95$ & 15.3 & 0.60 \\
\hline PAR 2 & 14 & $6 / 7 / 95$ & 15.0 & 0.60 \\
\hline PAR 2 & 0 & $6 / 27 / 95$ & 29.9 & 8.10 \\
\hline PAR 2 & 1 & $6 / 27 / 95$ & 29.9 & 7.48 \\
\hline PAR 2 & 2 & $6 / 27 / 95$ & 29.6 & 6.82 \\
\hline PAR 2 & 3 & $6 / 27 / 95$ & 28.7 & 3.06 \\
\hline PAR 2 & 4 & $6 / 27 / 95$ & 25.5 & 0.32 \\
\hline PAR 2 & 5 & $6 / 27 / 95$ & 20.9 & 0.42 \\
\hline PAR 2 & 6 & $6 / 27 / 95$ & 17.6 & 0.47 \\
\hline PAR 2 & 7 & $6 / 27 / 95$ & 16.8 & 0.42 \\
\hline PAR 2 & 8 & $6 / 27 / 95$ & 16.2 & 0.45 \\
\hline PAR 2 & 9 & $6 / 27 / 95$ & 15.9 & 0.43 \\
\hline PAR 2 & 10 & $6 / 27 / 95$ & 15.6 & 0.44 \\
\hline PAR 2 & 11 & $6 / 27 / 95$ & 15.3 & 0.47 \\
\hline PAR 2 & 0 & $7 / 11 / 95$ & 29.9 & 8.10 \\
\hline PAR 2 & 1 & $7 / 11 / 95$ & 29.9 & 7.48 \\
\hline PAR 2 & 2 & $7 / 11 / 95$ & 29.6 & 6.82 \\
\hline
\end{tabular}


Table A-2 (continued). Sample location: PAR 2.

\begin{tabular}{|c|c|c|c|c|}
\hline Location & Depth (meters) & Sample date & Temperature $\left({ }^{\circ} \mathrm{C}\right)$ & $\mathrm{DO}(\mathrm{mg} / \mathrm{L})$ \\
\hline & - & n- & - & a \\
\hline PAR 2 & 3 & $7 / 11 / 95$ & 28.7 & 3.06 \\
\hline PAR 2 & 4 & $7 / 11 / 95$ & 25.5 & 0.32 \\
\hline PAR 2 & 5 & $7 / 11 / 95$ & 20.9 & 0.42 \\
\hline PAR 2 & 6 & $7 / 11 / 95$ & 17.6 & 0.47 \\
\hline PAR 2 & 7 & $7 / 11 / 95$ & 16.8 & 0.42 \\
\hline PAR 2 & 8 & $7 / 11 / 95$ & 16.2 & 0.45 \\
\hline PAR 2 & 9 & $7 / 11 / 95$ & 15.9 & 0.43 \\
\hline PAR 2 & 10 & $7 / 11 / 95$ & 15.6 & 0.44 \\
\hline PAR 2 & 11 & $7 / 11 / 95$ & 15.3 & 0.47 \\
\hline PAR 2 & 0 & $7 / 25 / 95$ & 30.6 & 6.05 \\
\hline PAR 2 & 1 & $7 / 25 / 95$ & 29.8 & 5.38 \\
\hline PAR 2 & 2 & $7 / 25 / 95$ & 29.7 & 5.43 \\
\hline PAR 2 & 3 & $7 / 25 / 95$ & 29.6 & 5.12 \\
\hline PAR 2 & 4 & $7 / 25 / 95$ & 29.2 & 2.45 \\
\hline PAR 2 & 5 & $7 / 25 / 95$ & 26.9 & 0.31 \\
\hline PAR 2 & 6 & $7 / 25 / 95$ & 23.8 & 0.36 \\
\hline PAR 2 & 7 & $7 / 25 / 95$ & 20.3 & 0.41 \\
\hline PAR 2 & 8 & $7 / 25 / 95$ & 19.2 & 0.42 \\
\hline PAR 2 & 9 & $7 / 25 / 95$ & 17.9 & 0.44 \\
\hline PAR 2 & 10 & $7 / 25 / 95$ & 17.2 & 0.50 \\
\hline PAR 2 & 11 & $7 / 25 / 95$ & 16.7 & 0.46 \\
\hline PAR 2 & 12 & $7 / 25 / 95$ & 16.4 & 0.48 \\
\hline PAR 2 & $\overline{13}$ & $7 / 25 / 95$ & 16.2 & 0.50 \\
\hline PAR 2 & 14 & $7 / 25 / 95$ & 16.2 & 0.49 \\
\hline PAR 2 & 15 & $7 / 25 / 95$ & 16.2 & 0.45 \\
\hline PAR 2 & 0 & $8 / 22 / 95$ & 29.4 & 6.11 \\
\hline PAR 2 & $\overline{1}$ & $8 / 22 / 95$ & 29.2 & 5.92 \\
\hline PAR 2 & 2 & $8 / 22 / 95$ & 29.1 & 5.41 \\
\hline PAR 2 & 3 & $8 / 22 / 95$ & 29.0 & 5.48 \\
\hline PAR 2 & 4 & $8 / 22 / 95$ & 28.7 & 5.42 \\
\hline PAR 2 & 5 & $8 / 22 / 95$ & 28.7 & 2.36 \\
\hline PAR 2 & 6 & $8 / 22 / 95$ & 25.8 & 0.41 \\
\hline PAR 2 & 7 & $8 / 22 / 95$ & 22.7 & 0.45 \\
\hline PAR 2 & 8 & $8 / 22 / 95$ & 19.6 & 0.46 \\
\hline PAR 2 & 9 & $8 / 22 / 95$ & 18.7 & 0.48 \\
\hline PAR 2 & 10 & $8 / 22 / 95$ & 18.3 & 0.48 \\
\hline PAR 2 & 11 & $8 / 22 / 95$ & 18.1 & 0.50 \\
\hline PAR 2 & 12 & $8 / 22 / 95$ & 16.9 & 0.49 \\
\hline$\overline{\text { PAR } 2}$ & 13 & $8 / 22 / 95$ & 16.8 & 0.45 \\
\hline PAR 2 & 14 & $8 / 22 / 95$ & 16.5 & 0.43 \\
\hline PAR 2 & 15 & $8 / 22 / 95$ & 16.5 & 0.46 \\
\hline PAR 2 & 16 & $8 / 22 / 95$ & 16.4 & 0.44 \\
\hline PAR 2 & 0 & $9 / 7 / 95$ & 27.5 & 6.88 \\
\hline PAR 2 & 1 & $9 / 7 / 95$ & 27.3 & 6.73 \\
\hline PAR 2 & 2 & $9 / 7 / 95$ & 27.3 & 6.95 \\
\hline
\end{tabular}


Table A-2 (continued). Sample location: PAR 2.

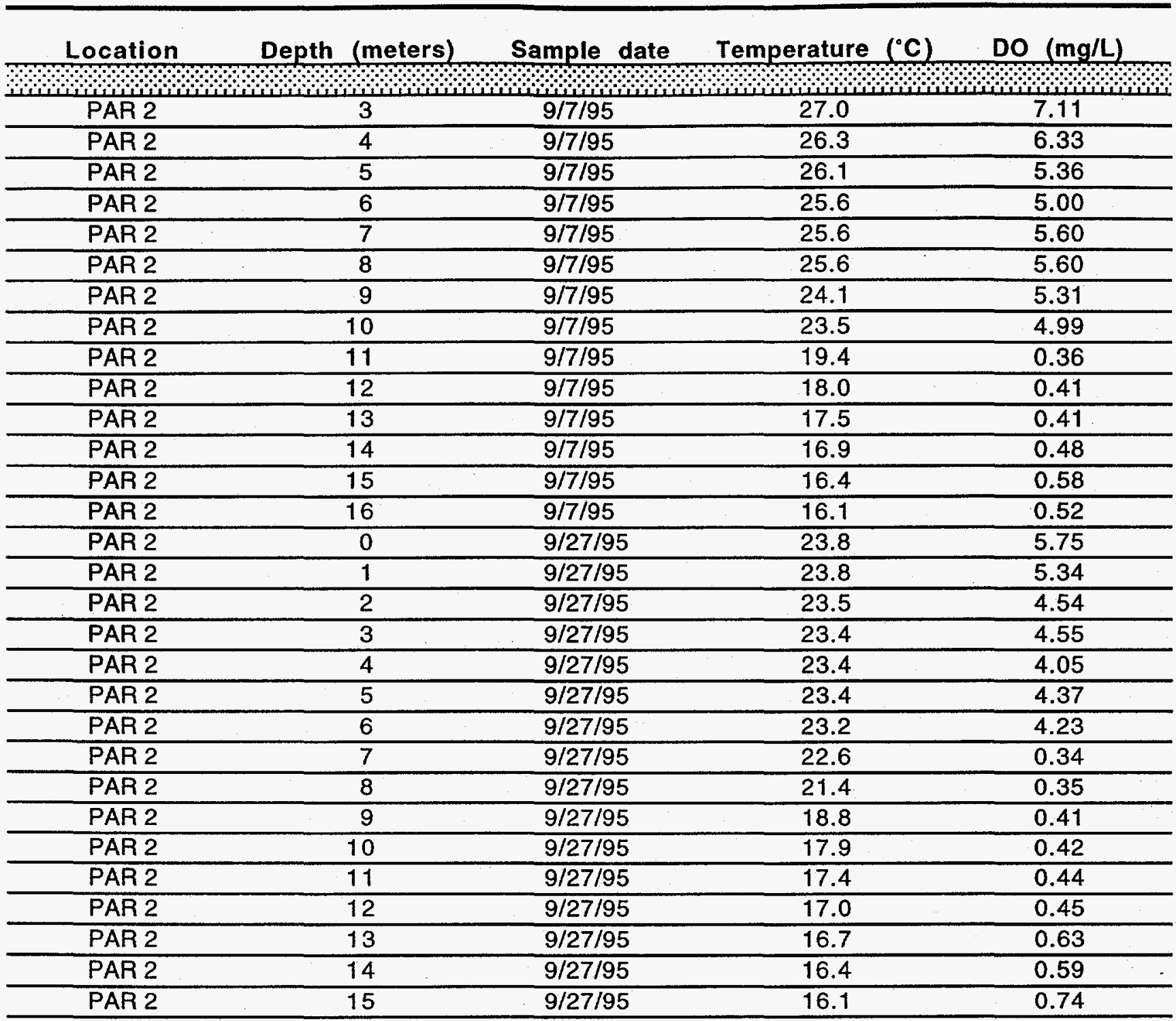


Table A-3. Sample location: PAR 3.

\begin{tabular}{|c|c|c|c|c|}
\hline Location & Depth (meters) & Sample date & Temperature $\left({ }^{\circ} \mathrm{C}\right)$ & DO (mg/L) \\
\hline n & - & Ha & n+m & and \\
\hline PAR 3 & 0 & $2 / 14 / 95$ & 16.1 & 5.80 \\
\hline PAR 3 & 1 & $2 / 14 / 95$ & 14.6 & 6.90 \\
\hline PAR 3 & 2 & $2 / 14 / 95$ & 14.4 & 6.40 \\
\hline PAR 3 & 3 & $2 / 14 / 95$ & 14.5 & 6.00 \\
\hline PAR 3 & 4 & $2 / 14 / 95$ & 14.0 & 5.30 \\
\hline PAR 3 & 0 & $2 / 21 / 95$ & 10.6 & 9.90 \\
\hline PAR 3 & 1 & $2 / 21 / 95$ & 10.0 & 9.90 \\
\hline PAR 3 & 2 & $2 / 21 / 95$ & 9.7 & 10.30 \\
\hline PAR 3 & 3 & $2 / 21 / 95$ & 9.7 & 9.60 \\
\hline PAR 3 & 4 & $2 / 21 / 95$ & 9.7 & 9.40 \\
\hline PAR 3 & 5 & $2 / 21 / 95$ & 9.7 & 9.80 \\
\hline PAR 3 & 6 & $2 / 21 / 95$ & 10.7 & 9.70 \\
\hline PAR 3 & 0 & $3 / 7 / 95$ & 15.8 & 9.60 \\
\hline PAR 3 & 1 & $3 / 7 / 95$ & 15.6 & 9.40 \\
\hline PAR 3 & 2 & $3 / 7 / 95$ & 15.4 & 9.40 \\
\hline PAR 3 & 3 & $3 / 7 / 95$ & 15.0 & 9.70 \\
\hline PAR 3 & 4 & $3 / 7 / 95$ & 14.8 & 9.90 \\
\hline PAR 3 & 5 & $3 / 7 / 95$ & 12.7 & 8.60 \\
\hline PAR 3 & 6 & $3 / 7 / 95$ & 12.0 & 10.10 \\
\hline PAR 3 & 7 & $3 / 7 / 95$ & 11.9 & 7.80 \\
\hline PAR 3 & 0 & $3 / 21 / 95$ & 16.1 & 9.40 \\
\hline PAR 3 & 1 & $3 / 21 / 95$ & 16.1 & 9.50 \\
\hline PAR 3 & 2 & $3 / 21 / 95$ & 16.0 & 9.30 \\
\hline PAR 3 & 3 & $3 / 21 / 95$ & 16.0 & 9.10 \\
\hline PAR 3 & 4 & $3 / 21 / 95$ & 15.3 & 8.60 \\
\hline PAR 3 & 5 & $3 / 21 / 95$ & 14.9 & 7.80 \\
\hline PAR 3 & 6 & $3 / 21 / 95$ & 14.8 & 8.30 \\
\hline PAR 3 & 7 & $3 / 21 / 95$ & 14.5 & 6.90 \\
\hline PAR 3 & 8 & $3 / 21 / 95$ & 12.8 & 5.90 \\
\hline PAR 3 & 0 & $4 / 4 / 95$ & 18.1 & 8.50 \\
\hline PAR 3 & 1 & $4 / 4 / 95$ & 18.1 & 8.10 \\
\hline PAR 3 & 2 & $4 / 4 / 95$ & 18.1 & $\overline{8.00}$ \\
\hline PAR 3 & $\overline{3}$ & $4 / 4 / 95$ & 18.1 & 8.00 \\
\hline PAR 3 & 4 & $4 / 4 / 95$ & 17.9 & 7.60 \\
\hline PAR 3 & 5 & $4 / 4 / 95$ & 17.6 & 7.60 \\
\hline PAR 3 & 6 & $4 / 4 / 95$ & 17.4 & 7.30 \\
\hline PAR 3 & 7 & $4 / 4 / 95$ & 16.6 & 4.20 \\
\hline PAR 3 & 8 & $4 / 4 / 95$ & 14.8 & 2.10 \\
\hline PAR 3 & 0 & $4 / 26 / 95$ & 24.4 & 5.50 \\
\hline PAR 3 & 1 & $4 / 26 / 95$ & 23.3 & 5.40 \\
\hline PAR 3 & 2 & $4 / 26 / 95$ & 21.9 & 4.60 \\
\hline PAR 3 & 3 & $4 / 26 / 95$ & 21.3 & 4.50 \\
\hline PAR 3 & 4 & $4 / 26 / 95$ & 21.2 & 1.90 \\
\hline PAR 3 & 5 & $4 / 26 / 95$ & 18.9 & 1.70 \\
\hline PAR 3 & 6 & $4 / 26 / 95$ & 17.7 & 1.40 \\
\hline
\end{tabular}


Table A-3 (continued). Sample location: PAR 3.

\section{Location}

\section{W}

$\frac{\text { PAR }}{\text { PAR } 3}$

PAR 3

PAR 3

PAR 3

PAR 3

PAR 3

PAR 3

PAR 3

PAR 3

PAR 3

PAR 3

PAR 3

PAR 3

PAR 3

PAR 3

PAR 3

PAR 3

PAR 3

PAR 3

PAR 3

PAR 3

PAR 3

PAR 3

PAR 3

PAR 3

PAR 3

PAR 3

PAR 3

PAR 3

PAR 3

PAR 3

PAR 3

PAR 3

PAR 3

PAR 3

PAR 3

PAR 3

PAR 3

PAR 3

PAR 3

PAR 3

PAR 3

PAR 3

PAR 3

PAR 3
Depth (meters)

Sample date

Temperature ( $\left.{ }^{\circ} \mathrm{C}\right)$

DO (mg/L)

$4 / 26 / 95$

4/26/95

$5 / 10 / 95$

$5 / 10 / 95$

$5 / 10 / 95$

$5 / 10 / 95$

$5 / 10 / 95$

$5 / 10 / 95$

$5 / 10 / 95$

$5 / 10 / 95$

$5 / 10 / 95$

$5 / 24 / 95$

$5 / 24 / 95$

$5 / 24 / 95$

$5 / 24 / 95$

$5 / 24 / 95$

$5 / 24 / 95$

$5 / 24 / 95$

$5 / 24 / 95$

$5 / 24 / 95$

$6 / 7 / 95$

$6 / 7 / 95$

$6 / 7 / 95$

$6 / 7 / 95$

$6 / 7 / 95$

$6 / 7 / 95$

$6 / 7 / 95$

$6 / 7 / 95$

$6 / 7 / 95$

$6 / 27 / 95$

$6 / 27 / 95$

$6 / 27 / 95$

$6 / 27 / 95$

$6 / 27 / 95$

$6 / 27 / 95$

$6 / 27 / 95$

$6 / 27 / 95$

$6 / 27 / 95$

$7 / 11 / 95$

$7 / 11 / 95$

$7 / 11 / 95$

$7 / 11 / 95$

$7 / 11 / 95$

$7 / 11 / 95$

$7 / 11 / 95$
16.4

15.8

24.9

24.8

24.8

24.2

23.4

23.1

22.5

17.8

16.0

29.6

27.5

25.8

25.3

24.6

23.8

19.8

18.3

16.2

26.9

26.9

26.7

25.8

25.7

25.5

25.2

22.3

19.1

30.3

28.7

28.4

26.6

25.1

24.7

24.0

23.5

21.4

31.4

31.2

29.4

27.5

25.5

23.1

19.8
1.40

1.40

8.20

7.70

7.60

7.40

5.50

4.90

3.70

0.90

0.90

6.90

6.10

5.10

4.50

2.23

0.37

0.39

0.44

0.52

6.10

5.90

5.30

4.90

4.30

3.70

2.80

0.59

0.74

7.06

7.47

5.59

5.18

3.68

1.68

1.22

0.77

0.65

7.47

7.87

4.43

0.19

0.52

0.61

0.70 
Table A-3 (continued). Sample location: PAR 3.

\begin{tabular}{|c|c|c|c|c|}
\hline Location & Depth (meters) & Sample date & Temperature $\left({ }^{\circ} \mathrm{C}\right)$ & $\mathrm{DO}(\mathrm{mg} / \mathrm{L})$ \\
\hline \multicolumn{5}{|l|}{ n- } \\
\hline PAR 3 & 0 & $7 / 25 / 95$ & 31.4 & 7.47 \\
\hline PAR 3 & 1 & $7 / 25 / 95$ & 31.2 & 7.87 \\
\hline PAR 3 & 2 & $7 / 25 / 95$ & 29.4 & 4.43 \\
\hline PAR 3 & 3 & $7 / 25 / 95$ & 27.5 & 0.19 \\
\hline PAR 3 & 4 & $7 / 25 / 95$ & 25.5 & 0.52 \\
\hline PAR 3 & 5 & $7 / 25 / 95$ & 23.1 & 0.61 \\
\hline PAR 3 & 6 & $7 / 25 / 95$ & 19.8 & 0.70 \\
\hline PAR 3 & 0 & $8 / 22 / 95$ & 30.6 & 5.51 \\
\hline PAR 3 & 1 & $8 / 22 / 95$ & 30.3 & 4.97 \\
\hline PAR 3 & 2 & $8 / 22 / 95$ & 30.2 & 4.39 \\
\hline PAR 3 & 3 & $8 / 22 / 95$ & 30.1 & 3.61 \\
\hline PAR 3 & 4 & $8 / 22 / 95$ & 28.6 & 1.51 \\
\hline PAR 3 & 5 & $8 / 22 / 95$ & 25.9 & 0.39 \\
\hline PAR 3 & 6 & $8 / 22 / 95$ & 23.8 & 0.44 \\
\hline PAR 3 & 7 & $8 / 22 / 95$ & 21.3 & 0.46 \\
\hline PAR 3 & 8 & $8 / 22 / 95$ & 19.1 & 0.52 \\
\hline PAR 3 & 0 & $9 / 7 / 95$ & 29.7 & 5.40 \\
\hline PAR 3 & 1 & $9 / 7 / 95$ & 29.4 & 5.10 \\
\hline PAR 3 & 2 & $9 / 7 / 95$ & 28.6 & 4.80 \\
\hline PAR 3 & 3 & $9 / 7 / 95$ & 27.2 & 3.90 \\
\hline PAR 3 & 4 & $9 / 7 / 95$ & 26.7 & 1.66 \\
\hline PAR 3 & 5 & $9 / 7 / 95$ & 24.6 & 0.55 \\
\hline PAR 3 & 6 & $9 / 7 / 95$ & 23.7 & 0.48 \\
\hline PAR 3 & 7 & $9 / 7 / 95$ & 20.5 & 0.51 \\
\hline PAR 3 & 8 & $9 / 7 / 95$ & 20.2 & 0.44 \\
\hline PAR 3 & 0 & $9 / 27 / 95$ & 26.0 & 6.52 \\
\hline PAR 3 & 1 & $9 / 27 / 95$ & 24.5 & 5.86 \\
\hline PAR 3 & 2 & $9 / 27 / 95$ & 23.8 & 3.37 \\
\hline PAR 3 & 3 & $9 / 27 / 95$ & 23.5 & 1.57 \\
\hline PAR 3 & 4 & $9 / 27 / 95$ & 23.4 & 2.08 \\
\hline PAR 3 & 5 & $9 / 27 / 95$ & 23.3 & 2.02 \\
\hline PAR 3 & 6 & $9 / 27 / 95$ & 23.2 & 1.83 \\
\hline
\end{tabular}


Table A-4. Sample location: PAR 4.

\begin{tabular}{|c|c|c|c|c|}
\hline Location & Depth (meters) & Sample date & Temperature $\left({ }^{\circ} \mathrm{C}\right)$ & DO (mg/L) \\
\hline \multicolumn{5}{|c|}{ की } \\
\hline PAR 4 & 0 & $2 / 14 / 95$ & 16.8 & 6.00 \\
\hline PAR 4 & 1 & $2 / 14 / 95$ & 17.0 & 5.30 \\
\hline PAR 4 & 2 & $2 / 14 / 95$ & 16.3 & 4.50 \\
\hline PAR 4 & 3 & $2 / 14 / 95$ & 16.7 & 4.30 \\
\hline PAR 4 & 4 & $2 / 14 / 95$ & 16.9 & 2.80 \\
\hline PAR 4 & 5 & $2 / 14 / 95$ & 16.3 & 2.40 \\
\hline PAR 4 & 0 & $2 / 21 / 95$ & 11.0 & 10.30 \\
\hline PAR 4 & 1 & $2 / 21 / 95$ & 10.5 & 9.40 \\
\hline PAR 4 & 2 & $2 / 21 / 95$ & 10.3 & 9.70 \\
\hline PAR 4 & 3 & $2 / 21 / 95$ & 10.3 & 9.70 \\
\hline PAR 4 & 4 & $2 / 21 / 95$ & 10.2 & 9.50 \\
\hline PAR 4 & 5 & $2 / 21 / 95$ & 10.2 & 9.80 \\
\hline PAR 4 & 6 & $2 / 21 / 95$ & 10.7 & 10.20 \\
\hline PAR 4 & 0 & $3 / 7 / 95$ & 14.7 & 9.80 \\
\hline PAR 4 & 1 & $3 / 7 / 95$ & 14.7 & 9.60 \\
\hline PAR 4 & 2 & $3 / 7 / 95$ & 14.6 & 9.90 \\
\hline PAR 4 & 3 & $3 / 7 / 95$ & 14.6 & 9.60 \\
\hline PAR 4 & 4. & $3 / 7 / 95$ & 14.6 & 9.70 \\
\hline PAR 4 & 5 & $3 / 7 / 95$ & 14.5 & 9.50 \\
\hline PAR 4 & 6 & $3 / 7 / 95$ & 14.5 & 9.90 \\
\hline PAR 4 & 7 & $3 / 7 / 95$ & 13.9 & 8.60 \\
\hline PAR 4 & 0 & $3 / 21 / 95$ & 17.3 & 9.60 \\
\hline PAR 4 & $T$ & $3 / 21 / 95$ & 17.1 & 9.60 \\
\hline PAR 4 & 2 & $3 / 21 / 95$ & 17.1 & 9.60 \\
\hline$\overline{\text { PAR } 4}$ & $\overline{3}$ & $3 / 21 / 95$ & 15.8 & 9.10 \\
\hline PAR 4 & 4 & $3 / 21 / 95$ & 15.1 & 8.90 \\
\hline PAR 4 & 5 & $3 / 21 / 95$ & 14.4 & 8.40 \\
\hline PAR 4 & 6 & $3 / 21 / 95$ & 13.7 & 5.20 \\
\hline PAR 4 & 7 & $3 / 21 / 95$ & 13.3 & 5.50 \\
\hline PAR 4 & 8 & $3 / 21 / 95$ & 13.1 & 5.70 \\
\hline$\overline{P A R} 4$ & 0 & $4 / 4 / 95$ & 18.4 & 8.10 \\
\hline PAR 4 & 1 & $4 / 4 / 95$ & 18.4 & 7.70 \\
\hline$\overline{\text { PAR } 4}$ & 2 & $4 / 4 / 95$ & 18.4 & 7.90 \\
\hline PAR 4 & 3 & $4 / 4 / 95$ & 18.3 & 7.70 \\
\hline PAR 4 & 4 & $4 / 4 / 95$ & 18.2 & 7.30 \\
\hline PAR 4 & 5 & $4 / 4 / 95$ & 16.9 & 2.10 \\
\hline PAR 4 & 6 & $4 / 4 / 95$ & 13.5 & 3.30 \\
\hline PAR 4 & 7 & $4 / 4 / 95$ & 13.5 & 3.30 \\
\hline PAR 4 & 8 & $4 / 4 / 95$ & 13.5 & 3.30 \\
\hline PAR 4 & 0 & $4 / 26 / 95$ & 23.2 & 4.70 \\
\hline PAR 4 & 1 & $4 / 26 / 95$ & 22.6 & 4.70 \\
\hline PAR 4 & 2 & $4 / 26 / 95$ & 22.3 & 4.40 \\
\hline PAR 4 & 3 & $4 / 26 / 95$ & 21.5 & 1.80 \\
\hline PAR 4 & 4 & $4 / 26 / 95$ & 19.9 & 1.80 \\
\hline PAR 4 & 5 & $4 / 26 / 95$ & 17.5 & 1.30 \\
\hline
\end{tabular}


Table A-4 (continued). Sample location: PAR 4.

\begin{tabular}{|c|c|c|c|c|}
\hline Location & Depth (meters) & Sample date & Temperature $\left({ }^{\circ} \mathrm{C}\right)$ & DO (mg/L) \\
\hline nn & on & an & & $\beta_{2}$ \\
\hline PAR 4 & 6 & $4 / 26 / 95$ & 15.9 & 1.30 \\
\hline PAR 4 & 7 & $4 / 26 / 95$ & 15.3 & 1.30 \\
\hline PAR 4 & 8 & $4 / 26 / 95$ & 14.8 & 1.30 \\
\hline PAR 4 & 0 & $5 / 10 / 95$ & 24.9 & 7.50 \\
\hline PAR 4 & 1 & $5 / 10 / 95$ & 24.9 & 7.90 \\
\hline PAR 4 & 2 & $5 / 10 / 95$ & 24.6 & 7.10 \\
\hline PAR 4 & 3 & $5 / 10 / 95$ & 24.4 & 6.90 \\
\hline PAR 4 & 4 & $5 / 10 / 95$ & 24.3 & 6.60 \\
\hline PAR 4 & 5 & $5 / 10 / 95$ & 24.2 & 6.50 \\
\hline PAR 4 & 6 & $5 / 10 / 95$ & 22.6 & 0.80 \\
\hline PAR 4 & 7 & $5 / 10 / 95$ & 15.7 & 0.90 \\
\hline PAR 4 & 8 & $5 / 10 / 95$ & 15.2 & 1.00 \\
\hline PAR 4 & 0 & $5 / 24 / 95$ & 26.1 & 6.20 \\
\hline PAR 4 & 1 & $5 / 24 / 95$ & 25.4 & 5.90 \\
\hline PAR 4 & 2 & $5 / 24 / 95$ & 25.3 & 5.30 \\
\hline PAR 4 & 3 & $5 / 24 / 95$ & 24.8 & 4.30 \\
\hline PAR 4 & 4 & $5 / 24 / 95$ & 20.7 & 0.61 \\
\hline PAR 4 & 5 & $5 / 24 / 95$ & 16.4 & 0.95 \\
\hline PAR 4 & 0 & $6 / 7 / 95$ & 27.0 & 7.20 \\
\hline PAR 4 & 1 & $6 / 7 / 95$ & 26.8 & 6.70 \\
\hline PAR 4 & 2 & $6 / 7 / 95$ & 26.5 & 6.20 \\
\hline PAR 4 & 3 & $6 / 7 / 95$ & 25.9 & 4.00 \\
\hline PAR 4 & 4 & $6 / 7 / 95$ & 25.1 & 1.30 \\
\hline PAR 4 & 5 & $6 / 7 / 95$ & 17.7 & 0.59 \\
\hline PAR 4 & 6 & $6 / 7 / 95$ & 16.9 & 0.63 \\
\hline PAR 4 & 7 & $6 / 7 / 95$ & 16.4 & 0.76 \\
\hline PAR 4 & 0 & $6 / 27 / 95$ & 33.9 & 6.82 \\
\hline PAR 4 & 1 & $6 / 27 / 95$ & 33.5 & 6.70 \\
\hline PAR 4 & 2 & $6 / 27 / 95$ & 30.0 & 6.80 \\
\hline PAR 4 & 3 & $6 / 27 / 95$ & 27.6 & 6.34 \\
\hline PAR 4 & 4 & $6 / 27 / 95$ & 26.0 & 0.24 \\
\hline PAR 4 & 5 & $6 / 27 / 95$ & 23.0 & 0.40 \\
\hline PAR 4 & 6 & $6 / 27 / 95$ & 19.5 & 0.37 \\
\hline PAR 4 & 7 & $6 / 27 / 95$ & 18.5 & 0.44 \\
\hline PAR 4 & 8 & $6 / 27 / 95$ & 16.3 & 0.36 \\
\hline PAR 4 & 0 & $7 / 11 / 95$ & 30.5 & 7.15 \\
\hline PAR 4 & 1 & $7 / 11 / 95$ & 30.5 & 6.50 \\
\hline PAR 4 & 2 & $7 / 11 / 95$ & 30.1 & 6.12 \\
\hline PAR 4 & 3 & $7 / 11 / 95$ & 27.6 & 0.13 \\
\hline PAR 4 & 4 & $7 / 11 / 95$ & 24.9 & 0.44 \\
\hline PAR 4 & 5 & $7 / 11 / 95$ & 23.2 & 0.47 \\
\hline PAR 4 & 6 & $7 / 11 / 95$ & 20.1 & 0.50 \\
\hline PAR 4 & 7 & $7 / 11 / 95$ & 17.7 & 0.64 \\
\hline PAR 4 & 0 & $7 / 25 / 95$ & 30.7 & 5.59 \\
\hline PAR 4 & 1 & $7 / 25 / 95$ & 30.5 & 5.10 \\
\hline
\end{tabular}


Table A-4 (continued). Sample location: PAR 4.

\begin{tabular}{|c|c|c|c|c|}
\hline Location & Depth (meters) & Sample date & Temperature ('c) & $\mathrm{DO}(\mathrm{mg} / \mathrm{L})$ \\
\hline O & $\cdots$ & \% & & \\
\hline PAR 4 & 2 & $7 / 25 / 95$ & 30.3 & 4.42 \\
\hline$\overline{P A R} 4$ & 3 & $7 / 25 / 95$ & 30.0 & 4.86 \\
\hline$\overline{\text { PAR } 4}$ & 4 & $7 / 25 / 95$ & 29.7 & 3.81 \\
\hline$\overline{\text { PAR } 4}$ & 5 & $7 / 25 / 95$ & 26.8 & 0.60 \\
\hline$\overline{\text { PAR } 4}$ & 6 & $7 / 25 / 95$ & 23.4 & 0.34 \\
\hline PAR 4 & 7 & $7 / 25 / 95$ & 20.4 & 0.37 \\
\hline PAR 4 & 8 & $7 / 25 / 95$ & 19.7 & 0.40 \\
\hline PAR 4 & 9 & $7 / 25 / 95$ & 17.8 & 0.48 \\
\hline PAR 4 & 0 & $8 / 22 / 95$ & 30.6 & 5.76 \\
\hline PAR 4 & 1 & $8 / 22 / 95$ & 30.5 & 5.40 \\
\hline PAR 4 & 2 & $8 / 22 / 95$ & 30.4 & 4.50 \\
\hline PAR 4 & 3 & $8 / 22 / 95$ & 30.4 & 4.70 \\
\hline PAR 4 & 4 & $8 / 22 / 95$ & 29.1 & 3.70 \\
\hline PAR 4 & 5 & $8 / 22 / 95$ & 26.5 & 0.56 \\
\hline PAR 4 & 6 & $8 / 22 / 95$ & 22.9 & 0.39 \\
\hline PAR 4 & 7 & $8 / 22 / 95$ & 20.8 & 0.40 \\
\hline PAR 4 & 8 & $8 / 22 / 95$ & 19.4 & 0.41 \\
\hline PAR 4 & 9 & $8 / 22 / 95$ & 18.1 & 0.45 \\
\hline PAR 4 & 0 & $9 / 7 / 95$ & 26.0 & 5.10 \\
\hline PAR 4 & 1 & $9 / 7 / 95$ & 25.3 & 4.60 \\
\hline PAR 4 & 2 & $9 / 7 / 95$ & 25.3 & 2.13 \\
\hline PAR 4 & 3 & $9 / 7 / 95$ & 25.1 & 2.07 \\
\hline PAR 4 & 4 & $9 / 7 / 95$ & 24.1 & 0.33 \\
\hline PAR 4 & 5 & $9 / 7 / 95$ & 23.8 & 0.30 \\
\hline PAR 4 & 6 & $9 / 7 / 95$ & 23.3 & 0.29 \\
\hline PAR 4 & 7 & $9 / 7 / 95$ & 22.4 & 0.38 \\
\hline PAR 4 & 8 & $9 / 7 / 95$ & 20.2 & 0.32 \\
\hline PAR 4 & 9 & $9 / 7 / 95$ & 20.0 & 0.30 \\
\hline PAR 4 & 0 & $9 / 27 / 95$ & 24.0 & 5.73 \\
\hline PAR 4 & 1 & $9 / 27 / 95$ & 23.8 & 5.06 \\
\hline PAR 4 & 2 & $9 / 27 / 95$ & 23.6 & 4.77 \\
\hline PAR 4 & 3 & $9 / 27 / 95$ & 23.0 & 3.12 \\
\hline$\overline{\text { PAR } 4}$ & 4 & $9 / 27 / 95$ & 22.3 & 0.62 \\
\hline PAR 4 & 5 & $9 / 27 / 95$ & 18.3 & 0.55 \\
\hline
\end{tabular}




\section{Appendix B}

\section{Analytical Results \\ Par Pond Stations 1-4 \\ February-September 1995}

Analytical results from the Par Pond refill water quality sampling are presented in this appendix. For each sampling location, the sampling date and the results of all laboratory analyses are presented. Included in the tables are any result or analytical qualifiers.
General Engineering Laboratories, Inc., performed all the analysis for this sampling project.

The abbreviation " $\mathrm{B}$ " in the tables represents a bottom sample; " $T$ " represents a surface sample. The following qualifiers are used with the results:

\begin{tabular}{|l|l|}
\hline Result Qualifiers \\
\hline Code & Description \\
\hline (Blank) & Data not remarked. Number should be interpreted exactly as reported. \\
\hline$J$ & The analytical result is an estimated quantity. \\
\hline$R$ & $\begin{array}{l}\text { Rejected because performance requirements in the sample or associated quality control } \\
\text { analyses were not met. The analyte may or may not be present. }\end{array}$ \\
\hline$U$ & Material analyzed for but not detected. Value reported is the sample quantification limit. \\
\hline
\end{tabular}

\begin{tabular}{|l|l|}
\hline Analysis Qualifiers \\
\hline Code & Description \\
\hline$Q$ & $\begin{array}{l}\text { Sample held beyond normal holding time. If holding time is exceeded by less than } 30 \text { days, } \\
\text { used with "J". If holding time is exceeded by more than } 30 \text { days, used with "R". }\end{array}$ \\
\hline$L$ & Analytical factor causing bias. The associated result may underestimate the true value. \\
\hline$H$ & Analytical factor causing bias. The associated result may overestimate the true value. \\
\hline$P$ & Analytical factor causing bias. The associated result may be of high variability. \\
\hline V & The analyte was detected in both the sample and associated method blank. \\
\hline
\end{tabular}


Table B-1. Sample location: Par top (T).

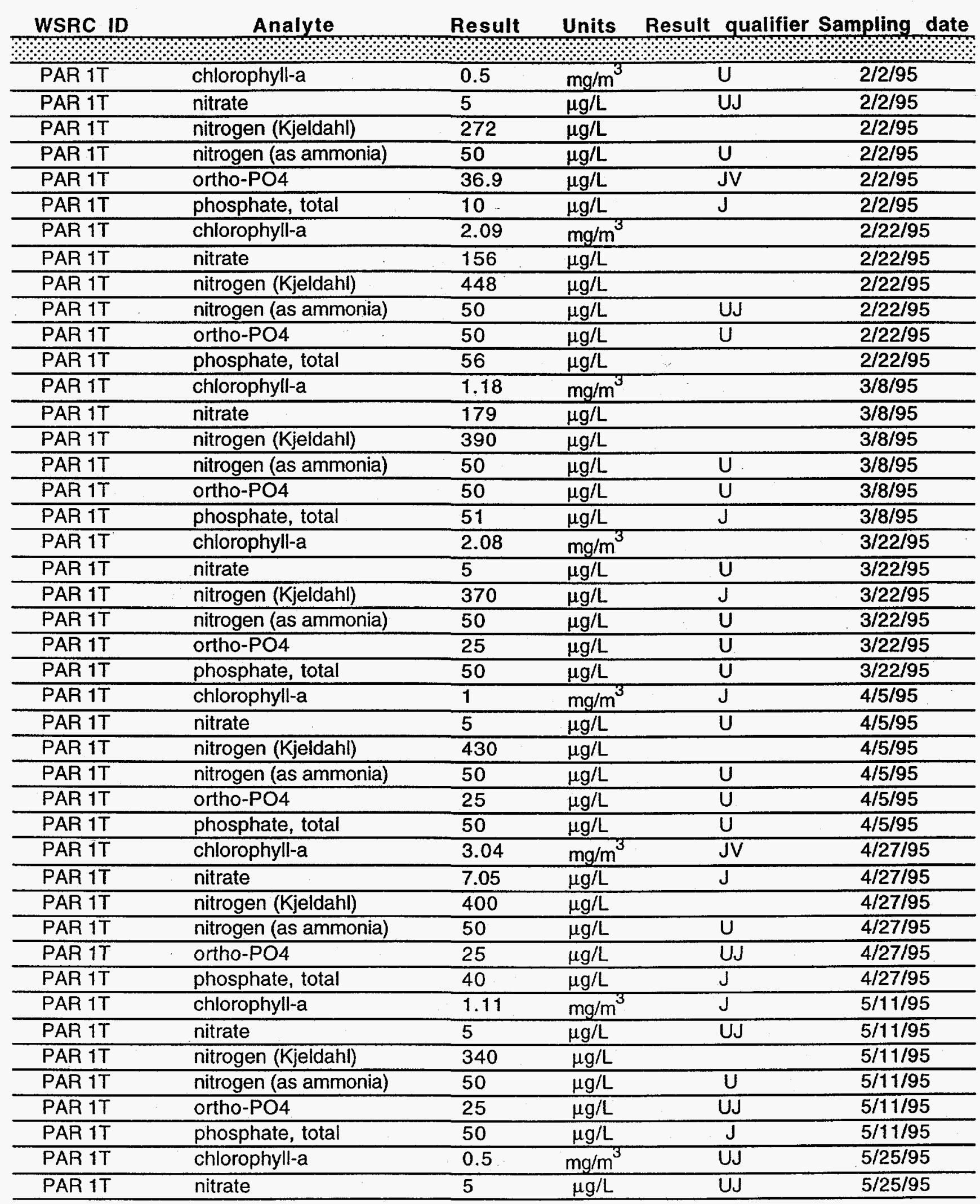


Table B-1 (continued). Sample location: Par 1 top (T).

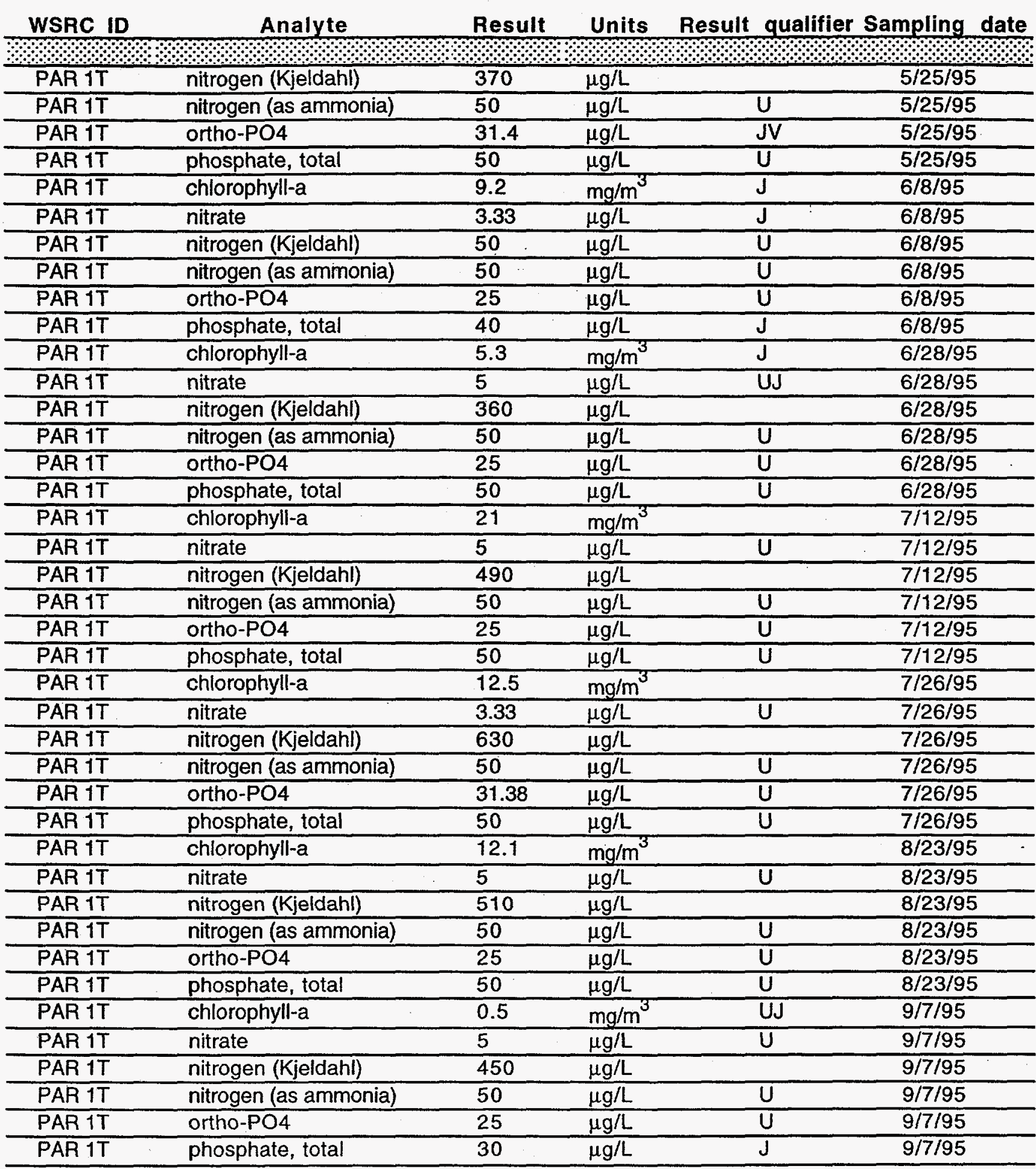


Table B-2. Sample location: Par 1 bottom (B).

WSRC ID

\begin{tabular}{|c|c|c|c|c|c|}
\hline PAR 1B & chlorophyll-a & 0.5 & $\mathrm{mg} / \mathrm{m}^{3}$ & U & $2 / 2 / 95$ \\
\hline PAR 1B & nitrate & 39.28 & $\mu g / L$ & US & $2 / 2 / 95$ \\
\hline PAR 1B & nitrogen (Kjeldahl) & 346 & $\mu \mathrm{g} / \mathrm{L}$ & & $2 / 2 / 95$ \\
\hline PAR 1B & nitrogen (as ammonia) & 50 & $\mu \mathrm{g} / \mathrm{L}$ & $\bar{U}$ & $2 / 2 / 95$ \\
\hline PAR 1B & PO4, total & 44.5 & $\mu \mathrm{g} / \mathrm{L}$ & JV & $2 / 2 / 95$ \\
\hline PAR 1B & phosphate, total & 25 & $\mu \mathrm{g} / \mathrm{L}$ & UJ & $2 / 2 / 95$ \\
\hline PAR 1B & chlorophyll-a & 1.94 & $\mathrm{mg} / \mathrm{m}^{3}$ & & $2 / 22 / 95$ \\
\hline PAR 1B & nitrate & 86.33 & $\mu \mathrm{g} / \mathrm{L}$ & & $2 / 22 / 95$ \\
\hline PAR 1B & nitrogen (Kjeldahl) & 443 & $\mu \mathrm{g} / \mathrm{L}$ & & $2 / 22 / 95$ \\
\hline PAR 1B & nitrogen (as ammonia) & 50 & $\mu \mathrm{g} / \mathrm{L}$ & UJ & $2 / 22 / 95$ \\
\hline PAR 1B & ortho-PO4 & 50 & $\mu \mathrm{g} / \mathrm{L}$ & U & $2 / 22 / 95$ \\
\hline PAR 1B & phosphate, total & 44 & $\mu \mathrm{g} / \mathrm{L}$ & $\mathrm{J}$ & $2 / 22 / 95$ \\
\hline PAR 1B & chlorophyll-a & 0.5 & $\mathrm{mg} / \mathrm{m}^{3}$ & $\mathrm{U}$ & $3 / 8 / 95$ \\
\hline PAR 1B & nitrate & 112.33 & $\mu \mathrm{g} / \mathrm{L}$ & & $3 / 8 / 95$ \\
\hline PAR 1B & nitrogen (Kjeldahl) & 231 & $\mu \mathrm{g} / \mathrm{L}$ & & $3 / 8 / 95$ \\
\hline PAR 1B & nitrogen (as ammonia) & 50 & $\mu \mathrm{g} / \mathrm{L}$ & $\bar{U}$ & $3 / 8 / 95$ \\
\hline PAR 1B & ortho-PO4 & 50 & $\mu \mathrm{g} / \mathrm{L}$ & $\bar{U}$ & $3 / 8 / 95$ \\
\hline PAR 1B & phosphate, total & 39 & $\mu \mathrm{g} / \mathrm{L}$ & $\mathrm{J}$ & $3 / 8 / 95$ \\
\hline PAR 1B & nitrate & 66.67 & $\mu \mathrm{g} / \mathrm{L}$ & & $3 / 22 / 95$ \\
\hline PAR 1B & nitrogen (Kjeldahl) & 290 & $\mu \mathrm{g} / \mathrm{L}$ & $J$ & $3 / 22 / 95$ \\
\hline PAR 1B & nitrogen (as ammonia) & 50 & $\mu \mathrm{g} / \mathrm{L}$ & $\bar{U}$ & $3 / 22 / 95$ \\
\hline PAR 1B & ortho-PO4 & 25 & $\mu \mathrm{g} / \mathrm{L}$ & $\bar{U}$ & $3 / 22 / 95$ \\
\hline PAR 1B & phosphate, total & 50 & $\mu \mathrm{g} / \mathrm{L}$ & $\bar{U}$ & $3 / 22 / 95$ \\
\hline PAR 1B & chlorophyll-a & 1.6 & $\mathrm{mg} / \mathrm{m}^{3}$ & & $4 / 5 / 95$ \\
\hline PAR 1B & nitrate & 28.96 & $\mu \mathrm{g} / \mathrm{L}$ & & $4 / 5 / 95$ \\
\hline PAR 1B & nitrogen (Kjeldahl) & 820 & $\mu \mathrm{g} / \mathrm{L}$ & & $4 / 5 / 95$ \\
\hline PAR 1B & nitrogen (as ammonia) & 50 & $\mu \mathrm{g} / \mathrm{L}$ & $\bar{U}$ & $4 / 5 / 95$ \\
\hline PAR 1B & ortho-PO4 & 20 & $\mu \mathrm{g} / \mathrm{L}$ & $J$ & $4 / 5 / 95$ \\
\hline PAR 1B & phosphate, total & 50 & $\mu \mathrm{g} / \mathrm{L}$ & $\bar{U}$ & $4 / 5 / 95$ \\
\hline PAR 1B & chlorophyll-a & 1.61 & $\mathrm{mg} / \mathrm{m}^{3}$ & JV & $4 / 27 / 95$ \\
\hline PAR 1B & nitrate & 34.3 & $\mu \mathrm{g} / \mathrm{L}$ & $J$ & $4 / 27 / 95$ \\
\hline PAR 1B & nitrogen (Kjeldahl) & 860 & $\mu \mathrm{g} / \mathrm{L}$ & & $4 / 27 / 95$ \\
\hline PAR 1B & nitrogen (as ammonia) & 150 & $\mu \mathrm{g} / \mathrm{L}$ & & $4 / 27 / 95$ \\
\hline PAR 1B & ortho-PO4 & 26 & $\mu \mathrm{g} / \mathrm{L}$ & $J$ & $4 / 27 / 95$ \\
\hline PAR 1B & phosphate, total & 80 & $\mu \mathrm{g} / \mathrm{L}$ & $J$ & $4 / 27 / 95$ \\
\hline PAR 1B & chlorophyll-a & 0.5 & $\mathrm{mg} / \mathrm{m}^{3}$ & UJ & $5 / 11 / 95$ \\
\hline PAR 1B & nitrate & 11.8 & $\mu \mathrm{g} / \mathrm{L}$ & $J$ & $5 / 11 / 95$ \\
\hline PAR 1B & nitrogen (Kjeldahl) & 650 & $\mu \mathrm{g} / \mathrm{L}$ & & $5 / 11 / 95$ \\
\hline PAR 1B & nitrogen (as ammonia) & 110 & $\mu \mathrm{g} / \mathrm{L}$ & & $5 / 11 / 95$ \\
\hline PAR 1B & ortho-PO4 & 28 & $\mu \mathrm{g} / \mathrm{L}$ & $\mathrm{J}$ & $5 / 11 / 95$ \\
\hline PAR 1B & phosphate, total & 250 & $\mu \mathrm{g} / \mathrm{L}$ & & $5 / 11 / 95$ \\
\hline PAR 1B & chlorophyll-a & 12.7 & $\mathrm{mg} / \mathrm{m}^{3}$ & $J$ & $5 / 25 / 95$ \\
\hline PAR 1B & nitrate & 3.33 & $\mu \mathrm{g} / \mathrm{L}$ & UJ & $5 / 25 / 95$ \\
\hline PAR 1B & nitrogen (Kjeldahl) & 870 & $\mu \mathrm{g} / \mathrm{L}$ & & $5 / 25 / 95$ \\
\hline
\end{tabular}


Table B-2 (continued). Sample location: Par 1 bottom (B).

\begin{tabular}{|c|c|c|c|c|c|c|}
\hline WSRC ID & Analyte & Result & Units & Result o & qualifier & Sampling \\
\hline & & & & & & \\
\hline PAR 1B & nitrogen (as ammonia) & 40 & $\mu \mathrm{g} / \mathrm{L}$ & $\mathrm{J}$ & J & $5 / 25 / 95$ \\
\hline PAR 1B & ortho-PO4 & 43.23 & $\mu g / L$ & & JV & $5 / 25 / 95$ \\
\hline PAR 1B & phosphate, total & 100 & $\mu g / L$ & $\mathrm{~J}$ & J & $5 / 25 / 95$ \\
\hline PAR 1B & chlorophyll-a & 19.7 & $\mathrm{mg} / \mathrm{m}^{3}$ & $J$ & $J$ & $6 / 8 / 95$ \\
\hline PAR 1B & nitrate & 9.44 & $\mu \mathrm{g} / \mathrm{L}$ & $J$ & J & $6 / 8 / 95$ \\
\hline PAR 1B & nitrogen (Kjeldahl) & 50 & $\mu \mathrm{g} / \mathrm{L}$ & $\bar{U}$ & $\bar{J}$ & $6 / 8 / 95$ \\
\hline PAR 1B & nitrogen (as ammonia) & 50 & $\mu \mathrm{g} / \mathrm{L}$ & $\mathrm{U}$ & $\bar{U}$ & $6 / 8 / 95$ \\
\hline PAR 1B & ortho-PO4 & 20 & $\mu \mathrm{g} / \mathrm{L}$ & $J$ & J & $6 / 8 / 95$ \\
\hline PAR 1B & phosphate, total & 50 & $\mu \mathrm{g} / \mathrm{L}$ & $\mathrm{J}$ & $\mathrm{J}$ & $6 / 8 / 95$ \\
\hline PAR 1B & chlorophyll-a & 59.7 & $\mathrm{mg} / \mathrm{m}^{3}$ & $\mathrm{~J}$ & $\mathrm{j}$ & $6 / 28 / 95$ \\
\hline PAR 1B & nitrate & 12.4 & $\mu \mathrm{g} / \mathrm{L}$ & $\mathrm{J}$ & $\mathrm{J}$ & $6 / 28 / 95$ \\
\hline PAR 1B & nitrogen (Kjeldahl) & 1180 & $\mu \mathrm{g} / \mathrm{L}$ & & & $6 / 28 / 95$ \\
\hline PAR 1B & nitrogen (as ammonia) & 50 & $\mu \mathrm{g} / \mathrm{L}$ & $\bar{U}$ & $\bar{U}$ & $6 / 28 / 95$ \\
\hline PAR 1B & ortho-PO4 & 58 & $\mu \mathrm{g} / \mathrm{L}$ & & & $6 / 28 / 95$ \\
\hline PAR 1B & phosphate, total & 100 & $\mu \mathrm{g} / \mathrm{L}$ & $J$ & J & $6 / 28 / 95$ \\
\hline PAR 1B & chlorophyll-a & 25 & $\mathrm{mg} / \mathrm{m}^{3}$ & & & $7 / 12 / 95$ \\
\hline PAR 1B & nitrate & 12.5 & $\mu \mathrm{g} / \mathrm{L}$ & & & $7 / 12 / 95$ \\
\hline PAR 1B & nitrogen (Kjeldahl) & 930 & $\mu \mathrm{g} / \mathrm{L}$ & & & $7 / 12 / 95$ \\
\hline PAR 1B & nitrogen (as ammonia) & 117.35 & $\mu \mathrm{g} / \mathrm{L}$ & & & $7 / 12 / 95$ \\
\hline PAR 1B & ortho-PO4 & 51 & $\mu \mathrm{g} / \mathrm{L}$ & & & $7 / 12 / 95$ \\
\hline PAR 1B & phosphate, total & 50 & $\mu \mathrm{g} / \mathrm{L}$ & $\mathrm{J}$ & J & $7 / 12 / 95$ \\
\hline PAR 1B & chlorophyll-a & 16.4 & $\mathrm{mg} / \mathrm{m}^{3}$ & & & $7 / 26 / 95$ \\
\hline PAR 1B & nitrate & 5 & $\mu g / L$ & & $\bar{U}$ & $7 / 26 / 95$ \\
\hline PAR 1B & nitrogen (Kjeldahl) & 590 & $\mu \mathrm{g} / \mathrm{L}$ & & & $7 / 26 / 95$ \\
\hline PAR 1B & nitrogen (as ammonia) & 50 & $\mu \mathrm{g} / \mathrm{L}$ & & $\bar{U}$ & $7 / 26 / 95$ \\
\hline PAR 1B & ortho-PO4 & 25 & $\mu \mathrm{g} / \mathrm{L}$ & & $\bar{U}$ & $7 / 26 / 95$ \\
\hline PAR 1B & phosphate, total & 50 & $\mu \mathrm{g} / \mathrm{L}$ & & $\bar{U}$ & $7 / 26 / 95$ \\
\hline PAR 1B & chlorophyll-a & 11.1 & $\mathrm{mg} / \mathrm{m}^{3}$ & & & $8 / 23 / 95$ \\
\hline PAR 1B & nitrate & 3.33 & $\mu \mathrm{g} / \mathrm{L}$ & & U & $8 / 23 / 95$ \\
\hline PAR 1B & nitrogen (Kjeldahl) & 710 & $\mu \mathrm{g} / \mathrm{L}$ & & & $8 / 23 / 95$ \\
\hline PAR 1B & nitrogen (as ammonia) & 50 & $\mu \mathrm{g} / \mathrm{L}$ & & $\bar{U}$ & $8 / 23 / 95$ \\
\hline PAR 1B & ortho-PO4 & 25 & $\mu g / L$ & & $\bar{U}$ & $8 / 23 / 95$ \\
\hline PAR 1B & phosphate, total & 70 & $\mu g / L$ & & $\mathrm{~J}$ & $8 / 23 / 95$ \\
\hline PAR 1B & chlorophyll-a & 3.81 & $\mathrm{mg} / \mathrm{m}^{3}$ & & $J$ & $9 / 7 / 95$ \\
\hline PAR 1B & nitrate & 27 & $\mu \mathrm{g} / \mathrm{L}$ & & & $9 / 7 / 95$ \\
\hline PAR 1B & nitrogen (Kjeldahl) & 2040 & $\mu \mathrm{g} / \mathrm{L}$ & & & $9 / 7 / 95$ \\
\hline PAR 1B & nitrogen (as ammonia) & 480 & $\mu \mathrm{g} / \mathrm{L}$ & & & $9 / 7 / 95$ \\
\hline PAR 1B & ortho-PO4 & 25 & $\mu \mathrm{g} / \mathrm{L}$ & & U & $9 / 7 / 95$ \\
\hline PAR 1B & phosphate, total & 140 & $\mu g / L$ & & & $9 / 7 / 95$ \\
\hline
\end{tabular}


Table B-3. Sample location: Par 2 top (T).

WSRC ID

\begin{tabular}{|c|c|c|c|c|c|}
\hline PAR 2T & chlorophyll-a & 1.92 & $\mathrm{mg} / \mathrm{m}^{3}$ & & $2 / 2 / 95$ \\
\hline PAR 2T & nitrate & 5 & $\mu \mathrm{g} / \mathrm{L}$ & UJ & $2 / 2 / 95$ \\
\hline PAR 2T & nitrogen (Kjeldahl) & 402 & $\mu \mathrm{g} / \mathrm{L}$ & & $2 / 2 / 95$ \\
\hline PAR 2T & nitrogen (as ammonia) & 50 & $\mu \mathrm{g} / \mathrm{L}$ & $\bar{U}$ & $2 / 2 / 95$ \\
\hline PAR 2T & ortho-PO4 & 50 & $\mu \mathrm{g} / \mathrm{L}$ & UV & $2 / 2 / 95$ \\
\hline PAR 2T & phosphate, total & 25 & $\mu \mathrm{g} / \mathrm{L}$ & UJ & $2 / 2 / 95$ \\
\hline PAR 2T & chlorophyll-a & 2.67 & $\mathrm{mg} / \mathrm{m}^{3}$ & & $2 / 22 / 95$ \\
\hline PAR 2T & nitrate & 87 & $\mu \mathrm{g} / \mathrm{L}$ & & $2 / 22 / 95$ \\
\hline PAR 2T & nitrogen (Kjeldahl) & 224 & $\mu \mathrm{g} / \mathrm{L}$ & & $2 / 22 / 95$ \\
\hline PAR 2T & nitrogen (as ammonia) & 50 & $\mu g / L$ & UJ & $2 / 22 / 95$ \\
\hline PAR 2T & ortho-PO4 & 50 & $\mu \mathrm{g} / \mathrm{L}$ & $\mathrm{U}$ & $2 / 22 / 95$ \\
\hline PAR 2T & phosphate, total & 12 & $\mu \mathrm{g} / \mathrm{L}$ & $J$ & $2 / 22 / 95$ \\
\hline PAR 2T & chlorophyll-a & 0.5 & $\mathrm{mg} / \mathrm{m}^{3}$ & $\mathrm{U}$ & $3 / 8 / 95$ \\
\hline PAR 2T & nitrate & 74 & $\mu g / L$ & & $3 / 8 / 95$ \\
\hline PAR 2T & nitrogen (Kjeldahl) & 209 & $\mu \mathrm{g} / \mathrm{L}$ & $\mathrm{V}$ & $3 / 8 / 95$ \\
\hline PAR 2T & nitrogen (as ammonia) & 50 & $\mu \mathrm{g} / \mathrm{L}$ & $\bar{U}$ & $3 / 8 / 95$ \\
\hline PAR 2T & ortho-PO4 & 50 & $\mu \mathrm{g} / \mathrm{L}$ & $\bar{U}$ & $3 / 8 / 95$ \\
\hline PAR 2T & phosphate, total & 25 & $\mu \mathrm{g} / \mathrm{L}$ & $\mathrm{U}$ & $3 / 8 / 95$ \\
\hline PAR 2T & chlorophyll-a & 2.29 & $\mathrm{mg} / \mathrm{m}^{3}$ & & $3 / 22 / 95$ \\
\hline PAR 2T & nitrate & 5 & $\mathrm{ug} / \mathrm{L}$ & $\mathrm{U}$ & $3 / 22 / 95$ \\
\hline PAR 2T & nitrogen (Kjeldahl) & 380 & $u g / L$ & $\mathrm{~J}$ & $3 / 22 / 95$ \\
\hline PAR 2T & nitrogen (as ammonia) & 50 & $\mathrm{Ug} / \mathrm{L}$ & $\bar{U}$ & $3 / 22 / 95$ \\
\hline PAR 2T & ortho-PO4 & 25 & $\mathrm{ug} / \mathrm{L}$ & $\mathrm{U}$ & $3 / 22 / 95$ \\
\hline PAR 2T & phosphate, total & 50 & $\mathrm{ug} / \mathrm{L}$ & $\bar{U}$ & $3 / 22 / 95$ \\
\hline PAR 2T & chlorophyll-a & 1.69 & $\mathrm{mg} / \mathrm{m}^{3}$ & & $4 / 5 / 95$ \\
\hline PAR 2T & nitrate & 5 & $\mathrm{ug} / \mathrm{L}$ & $\mathrm{U}$ & $4 / 5 / 95$ \\
\hline PAR 2T & nitrogen (Kjeldahl) & 520 & $\mathrm{ug} / \mathrm{L}$ & & $4 / 5 / 95$ \\
\hline PAR 2T & nitrogen (as ammonia) & 50 & $\mathrm{ug} / \mathrm{L}$ & $\mathrm{U}$ & $4 / 5 / 95$ \\
\hline PAR 2T & ortho-PO4 & 21.9 & $\mathrm{ug} / \mathrm{L}$ & & $4 / 5 / 95$ \\
\hline PAR 2T & phosphate, total & 50 & $\mathrm{ug} / \mathrm{L}$ & $\bar{U}$ & $4 / 5 / 95$ \\
\hline PAR 2T & chlorophyll-a & 4.58 & $\mathrm{mg} / \mathrm{m}^{3}$ & JV. & $4 / 27 / 95$ \\
\hline PAR 2T & nitrate & 16.2 & $\mu \mathrm{g} / \mathrm{L}$ & $J$ & $4 / 27 / 95$ \\
\hline PAR 2T & nitrogen (Kjeldahl) & 380 & $\mu \mathrm{g} / \mathrm{L}$ & & $4 / 27 / 95$ \\
\hline PAR 2T & nitrogen (as ammonia) & 50 & $\mu \mathrm{g} / \mathrm{L}$ & $\mathrm{U}$ & $4 / 27 / 95$ \\
\hline PAR 2T & ortho-PO4 & 25 & $\mu \mathrm{g} / \mathrm{L}$ & UJ & $4 / 27 / 95$ \\
\hline PAR 2T & phosphate, total & 50 & $\mu \mathrm{g} / \mathrm{L}$ & $\mathrm{U}$ & $4 / 27 / 95$ \\
\hline PAR 2T & chlorophyll-a & 1.58 & $\mathrm{mg} / \mathrm{m}^{3}$ & $\mathbf{J}$ & $5 / 11 / 95$ \\
\hline PAR 2T & nitrate & 5 & $\mu \mathrm{g} / \mathrm{L}$ & UJ & $5 / 11 / 95$ \\
\hline PAR 2T & nitrogen (Kjeldahl) & 250 & $\mu g / L$ & & $5 / 11 / 95$ \\
\hline PAR 2T & nitrogen (as ammonia) & 50 & $\mu \mathrm{g} / \mathrm{L}$ & $U$ & $5 / 11 / 95$ \\
\hline PAR 2T & ortho-PO4 & 25 & $\mu \mathrm{g} / \mathrm{L}$ & UJ & $5 / 11 / 95$ \\
\hline PAR 2T & phosphate, total & 550 & $\mu \mathrm{g} / \mathrm{L}$ & & $5 / 11 / 95$ \\
\hline PAR 2T & chlorophyll-a & 0.5 & $\mathrm{mg} / \mathrm{m}^{3}$ & UJ & $5 / 25 / 95$ \\
\hline PAR 2T & nitrate & 5 & $\mu \mathrm{g} / \mathrm{L}$ & UJ & $5 / 25 / 95$ \\
\hline
\end{tabular}


Table B-3 (continued). Sample location: Par 2 top (T).

\begin{tabular}{|c|c|c|c|c|c|}
\hline WSRC ID & Analyte & Result & Units & Result qualifier & Sampling date \\
\hline - & $\because \cdots$ & & m & ओ & की \\
\hline PAR 2T & nitrogen (Kjeldahl) & 350 & $\mu \mathrm{g} / \mathrm{L}$ & & $5 / 25 / 95$ \\
\hline PAR 2T & nitrogen (as ammonia) & 33.33 & $\mu g / L$ & $\bar{U}$ & $5 / 25 / 95$ \\
\hline PAR 2T & ortho-PO4 & 26 & $\mu \mathrm{g} / \mathrm{L}$ & JV & $5 / 25 / 95$ \\
\hline PAR 2T & phosphate, total & 50 & $\mu \mathrm{g} / \mathrm{L}$ & $\bar{U}$ & $5 / 25 / 95$ \\
\hline PAR 2T & chlorophyll-a & 12.5 & $\mathrm{mg} / \mathrm{m}^{3}$ & $J$ & $6 / 8 / 95$ \\
\hline PAR 2T & nitrate & 5 & $\mu g / L$ & UJ & $6 / 8 / 95$ \\
\hline PAR 2T & nitrogen (Kjeldahl) & 50 & $\mu \mathrm{g} / \mathrm{L}$ & $\bar{U}$ & $6 / 8 / 95$ \\
\hline PAR 2T & nitrogen (as ammonia) & 50 & $\mu \mathrm{g} / \mathrm{L}$ & $\bar{U}$ & $6 / 8 / 95$ \\
\hline PAR 2T & ortho-PO4 & 25 & $\mu \mathrm{g} / \mathrm{L}$ & $U$ & $6 / 8 / 95$ \\
\hline PAR 2T & phosphate, total & 50 & $\mu \mathrm{g} / \mathrm{L}$ & $\bar{U}$ & $6 / 8 / 95$ \\
\hline PAR 2T & chlorophyll-a & 7.4 & $\mathrm{mg} / \mathrm{m}^{3}$ & J & $6 / 28 / 95$ \\
\hline PAR 2T & nitrate & 5 & $\mu \mathrm{g} / \mathrm{L}$ & UJ & $6 / 28 / 95$ \\
\hline PAR 2T & nitrogen (Kjeldahl) & 450 & $\mu \mathrm{g} / \mathrm{L}$ & & $6 / 28 / 95$ \\
\hline PAR 2T & nitrogen (as ammonia) & 50 & $\mu \mathrm{g} / \mathrm{L}$ & $\mathrm{U}$ & $6 / 28 / 95$ \\
\hline PAR 2T & ortho-PO4 & 25 & $\mu \mathrm{g} / \mathrm{L}$ & $\bar{U}$ & $6 / 28 / 95$ \\
\hline PAR 2T & phosphate, total & 50 & $\mu \mathrm{g} / \mathrm{L}$ & $\bar{U}$ & $6 / 28 / 95$ \\
\hline PAR 2T & chlorophyll-a & 19.2 & $\mathrm{mg} / \mathrm{m}^{3}$ & & $7 / 12 / 95$ \\
\hline PAR 2T & nitrate & 5 & $\mu \mathrm{g} / \mathrm{L}$ & $\mathrm{U}$ & $7 / 12 / 95$ \\
\hline PAR 2T & nitrogen (Kjeldahl) & 490 & $\mu g / L$ & & $7 / 12 / 95$ \\
\hline PAR 2T & nitrogen (as ammonia) & 50 & $\mu \mathrm{g} / \mathrm{L}$ & $\bar{U}$ & $7 / 12 / 95$ \\
\hline PAR 2T & ortho-PO4 & 28 & $\mu \mathrm{g} / \mathrm{L}$ & $J$ & $7 / 12 / 95$ \\
\hline PAR 2T & phosphate, total & 30 & $\mu \mathrm{g} / \mathrm{L}$ & $\mathrm{J}$ & $7 / 12 / 95$ \\
\hline PAR 2T & chlorophyll-a & 14.6 & $\mathrm{mg} / \mathrm{m}^{3}$ & & $7 / 26 / 95$ \\
\hline PAR 2T & nitrate & 5 & $\mu \mathrm{g} / \mathrm{L}$ & $\bar{U}$ & $7 / 26 / 95$ \\
\hline PAR.2T & nitrogen (Kjeldahl) & 681.59 & $\mu \mathrm{g} / \mathrm{L}$ & & $7 / 26 / 95$ \\
\hline PAR 2T & nitrogen (as ammonia) & 33.33 & $\mu \mathrm{g} / \mathrm{L}$ & $\bar{U}$ & $7 / 26 / 95$ \\
\hline PAR 2T & ortho-PO4 & 35.12 & $\mu \mathrm{g} / \mathrm{L}$ & $\bar{U}$ & $7 / 26 / 95$ \\
\hline PAR 2T & phosphate, total & 33.33 & $\mu \mathrm{g} / \mathrm{L}$ & & $7 / 26 / 95$ \\
\hline PAR 2T & chlorophyll-a & 13.6 & $\mathrm{mg} / \mathrm{m}^{3}$ & & $8 / 23 / 95$ \\
\hline PAR 2T & nitrate & 5 & $\mu \mathrm{g} / \mathrm{L}$ & $\bar{U}$ & $8 / 23 / 95$ \\
\hline PAR 2T & nitrogen (Kjeldahl) & 453.69 & $\mu \mathrm{g} / \mathrm{L}$ & & $8 / 23 / 95$ \\
\hline PAR 2T & nitrogen (as ammonia) & 50 & $\mu \mathrm{g} / \mathrm{L}$ & $\mathrm{U}$ & $8 / 23 / 95$ \\
\hline PAR 2T & ortho-PO4 & 25 & $\mu \mathrm{g} / \mathrm{L}$ & $\mathrm{U}$ & $8 / 23 / 95$ \\
\hline PAR 2T & phosphate, total & 33.33 & $\mu \mathrm{g} / \mathrm{L}$ & & $8 / 23 / 95$ \\
\hline PAR 2T & chlorophyll-a & 5.59 & $\mathrm{mg} / \mathrm{m}^{3}$ & $J$ & $9 / 7 / 95$ \\
\hline PAR 2T & nitrate & 5 & $\mu g / L$ & $\bar{U}$ & $9 / 7 / 95$ \\
\hline PAR 2T & nitrogen (Kjeldahl) & 360 & $\mu g / L$ & & $9 / 7 / 95$ \\
\hline PAR 2T & nitrogen (as ammonia) & 50 & $\mu \mathrm{g} / \mathrm{L}$ & $\bar{U}$ & $9 / 7 / 95$ \\
\hline PAR 2T & ortho-PO4 & 25 & $\mu \mathrm{g} / \mathrm{L}$ & $\bar{U}$ & $9 / 7 / 95$ \\
\hline PAR 2T & phosphate, total & 50 & $\mu g / L$ & 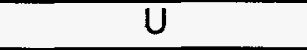 & $9 / 7 / 95$ \\
\hline
\end{tabular}


Table B-4 (continued). Sample location: Par 2 bottom (B).

WSRC ID PAR

\begin{tabular}{|c|c|c|c|c|c|}
\hline PAR 2B & nitrate & 5 & $\mu \mathrm{g} / \mathrm{L}$ & UJ & $5 / 25 / 95$ \\
\hline PAR 2B & nitrogen (Kjeldahl) & 480 & $\mu \mathrm{g} / \mathrm{L}$ & & $5 / 25 / 95$ \\
\hline PAR 2B & nitrogen (as ammonia) & 150 & $\mu \mathrm{g} / \mathrm{L}$ & & $5 / 25 / 95$ \\
\hline PAR 2B & ortho-PO4 & 29 & $\mu \mathrm{g} / \mathrm{L}$ & $\mathrm{JV}$ & $5 / 25 / 95$ \\
\hline PAR 2B & phosphate, total & 90 & $\mu \mathrm{g} / \mathrm{L}$ & $J$ & $5 / 25 / 95$ \\
\hline PAR 2B & chlorophyll-a & 5.4 & $\mathrm{mg} / \mathrm{m}^{3}$ & 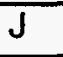 & $6 / 8 / 95$ \\
\hline PAR 2B & nitrate & 10.1 & $\mu \mathrm{g} / \mathrm{L}$ & $J$ & $6 / 8 / 95$ \\
\hline PAR 2B & nitrogen (Kjeldahl) & 50 & $\mu \mathrm{g} / \mathrm{L}$ & $\bar{U}$ & $6 / 8 / 95$ \\
\hline PAR 2B & nitrogen (as ammonia) & 200 & $\mu \mathrm{g} / \mathrm{L}$ & & $6 / 8 / 95$ \\
\hline PAR 2B & ortho-PO4 & 72 & $\mu \mathrm{g} / \mathrm{L}$ & & $6 / 8 / 95$ \\
\hline PAR 2B & phosphate, total & 80 & $\mu \mathrm{g} / \mathrm{L}$ & J & $6 / 8 / 95$ \\
\hline PAR 2B & chlorophyll-a & 19.8 & $\mathrm{mg} / \mathrm{m}^{3}$ & $\bar{J}$ & $6 / 28 / 95$ \\
\hline PAR 2B & nitrate & 10.4 & $\mu \mathrm{g} / \mathrm{L}$ & $\mathrm{J}$ & $6 / 28 / 95$ \\
\hline PAR 2B & nitrogen (Kjeldahl) & 330 & $\mu \mathrm{g} / \mathrm{L}$ & & $6 / 28 / 95$ \\
\hline PAR 2B & nitrogen (as ammonia) & 50 & $\mu \mathrm{g} / \mathrm{L}$ & $\bar{U}$ & $6 / 28 / 95$ \\
\hline PAR 2B & ortho-PO4 & 18 & $\mu \mathrm{g} / \mathrm{L}$ & $J$ & $6 / 28 / 95$ \\
\hline PAR 2B & phosphate, total & 50 & $\mu \mathrm{g} / \mathrm{L}$ & $\bar{U}$ & $6 / 28 / 95$ \\
\hline PAR 2B & chlorophyll-a & 29.3 & $\mathrm{mg} / \mathrm{m}^{3}$ & & $7 / 12 / 95$ \\
\hline PAR 2B & nitrate & 10.7 & $\mu \mathrm{g} / \mathrm{L}$ & & $7 / 12 / 95$ \\
\hline PAR 2B & nitrogen (Kjeldahl) & 540 & $\mu \mathrm{g} / \mathrm{L}$ & & $7 / 12 / 95$ \\
\hline PAR 2B & nitrogen (as ammonia) & 50 & $\mu \mathrm{g} / \mathrm{L}$ & $\mathrm{U}$ & $7 / 12 / 95$ \\
\hline PAR 2B & ortho-PO4 & 21 & $\mu \mathrm{g} / \mathrm{L}$ & $\mathrm{J}$ & $7 / 12 / 95$ \\
\hline PAR 2B & phosphate, total & 50 & $\mu \mathrm{g} / \mathrm{L}$ & $\bar{U}$ & $7 / 12 / 95$ \\
\hline PAR 2B & chlorophyll-a & 9.4 & $\mathrm{mg} / \mathrm{m}^{3}$ & & $7 / 26 / 95$ \\
\hline PAR 2B & nitrate & 5 & $\mu \mathrm{g} / \mathrm{L}$ & $\bar{U}$ & $7 / 26 / 95$ \\
\hline PAR 2B & nitrogen (Kjeldahl) & 470 & $\mu \mathrm{g} / \mathrm{L}$ & & $7 / 26 / 95$ \\
\hline PAR 2B & nitrogen (as ammonia) & 200 & $\mu \mathrm{g} / \mathrm{L}$ & & $7 / 26 / 95$ \\
\hline PAR 2B & ortho-PO4 & 57 & $\mu \mathrm{g} / \mathrm{L}$ & & $7 / 26 / 95$ \\
\hline PAR 2B & phosphate, total & 50 & $\mu \mathrm{g} / \mathrm{L}$ & $U$ & $7 / 26 / 95$ \\
\hline PAR 2B & chlorophyll-a & 12.2 & $\mathrm{mg} / \mathrm{m}^{3}$ & & $8 / 23 / 95$ \\
\hline PAR 2B & nitrate & 5 & $\mu \mathrm{g} / \mathrm{L}$ & $\mathrm{U}$ & $8 / 23 / 95$ \\
\hline PAR 2B & nitrogen (Kjeldahl) & 430 & $\mu \mathrm{g} / \mathrm{L}$ & & $8 / 23 / 95$ \\
\hline PAR 2B & nitrogen (as ammonia) & 50 & $\mu \mathrm{g} / \mathrm{L}$ & $\mathrm{U}$ & $8 / 23 / 95$ \\
\hline PAR 2B & ortho-PO4 & 25 & $\mu \mathrm{g} / \mathrm{L}$ & $\mathrm{U}$ & $8 / 23 / 95$ \\
\hline PAR 2B & phosphate, total & 50 & $\mu \mathrm{g} / \mathrm{L}$ & $\bar{U}$ & $8 / 23 / 95$ \\
\hline PAR 2B & chlorophyll-a & 5.98 & $\mathrm{mg} / \mathrm{m}^{3}$ & $\mathrm{~J}$ & $9 / 7 / 95$ \\
\hline PAR 2B & nitrate & 5 & $\mu \mathrm{g} / \mathrm{L}$ & $\mathrm{U}$ & $9 / 7 / 95$ \\
\hline PAR 2B & nitrogen (Kjeldahl) & 350 & $\mu \mathrm{g} / \mathrm{L}$ & & 9/7/95 \\
\hline PAR 2B & nitrogen (as ammonia) & 50 & $\mu \mathrm{g} / \mathrm{L}$ & $\mathrm{U}$ & $9 / 7 / 95$ \\
\hline PAR 2B & ortho-PO4 & 25 & $\mu \mathrm{g} / \mathrm{L}$ & $\mathrm{U}$ & 9/7/95 \\
\hline PAR 2B & phosphate, total & 50 & $\mu \mathrm{g} / \mathrm{L}$ & $\mathrm{U}$ & $9 / 7 / 95$ \\
\hline
\end{tabular}


Table B-5. Sample location: Par 3 top (T).

\begin{tabular}{|c|c|c|c|c|c|}
\hline WSRC ID & Analyte & Result & Units & Result qualifier & Sampling date \\
\hline \multicolumn{6}{|l|}{ all } \\
\hline PAR 3T & chlorophyll-a & 0.5 & $\mathrm{mg} / \mathrm{m}^{3}$ & U & $2 / 2 / 95$ \\
\hline PAR 3T & nitrate & 5 & $\mu \mathrm{g} / \mathrm{L}$ & UJ & $2 / 2 / 95$ \\
\hline PAR 3T & nitrogen (Kjeldahl) & 833 & $\mu \mathrm{g} / \mathrm{L}$ & & $2 / 2 / 95$ \\
\hline PAR 3T & nitrogen (as ammonia) & 50 & $\mu \mathrm{g} / \mathrm{L}$ & $\bar{U}$ & $2 / 2 / 95$ \\
\hline PAR 3T & ortho-PO4 & 166 & $\mu \mathrm{g} / \mathrm{L}$ & $\mathrm{V}$ & $2 / 2 / 95$ \\
\hline PAR 3T & phosphate, total & 25 & $\mu \mathrm{g} / \mathrm{L}$ & UJ & $2 / 2 / 95$ \\
\hline PAR 3T & chlorophyll-a & 0.5 & $\mathrm{mg} / \mathrm{m}^{3}$ & $\bar{U}$ & $2 / 22 / 95$ \\
\hline PAR 3T & nitrate & 108 & $\mu \mathrm{g} / \mathrm{L}$ & & $2 / 22 / 95$ \\
\hline PAR 3T & nitrogen (Kjeldahl) & 375 & $\mu \mathrm{g} / \mathrm{L}$ & & $2 / 22 / 95$ \\
\hline PAR 3T & nitrogen (as ammonia) & 50 & $\mu \mathrm{g} / \mathrm{L}$ & UJ & $2 / 22 / 95$ \\
\hline PAR 3T & ortho-PO4 & 50 & $\mu \mathrm{g} / \mathrm{L}$ & $\bar{U}$ & $2 / 22 / 95$ \\
\hline PAR 3T & phosphate, total & 25 & $\mu \mathrm{g} / \mathrm{L}$ & $\bar{U}$ & $2 / 22 / 95$ \\
\hline PAR 3T & chlorophyll-a & 0.5 & $\mathrm{mg} / \mathrm{m}^{3}$ & $\bar{U}$ & $3 / 8 / 95$ \\
\hline PAR 3T & nitrate & 56 & $\mu \mathrm{g} / \mathrm{L}$ & & $3 / 8 / 95$ \\
\hline PAR 3T & nitrogen (Kjeldahl) & 262 & $\mu \mathrm{g} / \mathrm{L}$ & & $3 / 8 / 95$ \\
\hline PAR 3T & nitrogen (as ammonia) & 50 & $\mu \mathrm{g} / \mathrm{L}$ & $\bar{U}$ & $3 / 8 / 95$ \\
\hline PAR 3T & ortho-PO4 & 50 & $\mu \mathrm{g} / \mathrm{L}$ & $\bar{U}$ & $3 / 8 / 95$ \\
\hline PAR 3T & phosphate, total & 13 & $\mu \mathrm{g} / \mathrm{L}$ & $\mathrm{J}$ & $3 / 8 / 95$ \\
\hline PAR 3T & chlorophyll-a & 1.54 & $\mathrm{mg} / \mathrm{m}^{3}$ & & $3 / 22 / 95$ \\
\hline PAR 3T & nitrate & 5 & $\mathrm{ug} / \mathrm{L}$ & $\bar{U}$ & $3 / 22 / 95$ \\
\hline PAR 3T & nitrogen (Kjeldahl) & 360 & $\mathrm{ug} / \mathrm{L}$ & $J$ & $3 / 22 / 95$ \\
\hline PAR 3T & nitrogen (as ammonia) & 50 & $\mathrm{ug} / \mathrm{L}$ & $\bar{U}$ & $3 / 22 / 95$ \\
\hline PAR 3T & ortho-PO4 & 25 & $\mathrm{ug} / \mathrm{L}$ & $\bar{U}$ & $3 / 22 / 95$ \\
\hline PAR 3T & phosphate, total & 50 & $\mathrm{ug} / \mathrm{L}$ & $\mathrm{U}$ & $3 / 22 / 95$ \\
\hline PAR 3T & chlorophyll-a & 1.89 & $\mathrm{mg} / \mathrm{m}^{3}$ & & $4 / 5 / 95$ \\
\hline PAR 3T & nitrate & 5 & $\mathrm{ug} / \mathrm{L}$ & $\bar{U}$ & $4 / 5 / 95$ \\
\hline PAR 3T & nitrogen (Kjeldahl) & 310 & $\mathrm{Ug} / \mathrm{L}$ & & $4 / 5 / 95$ \\
\hline PAR 3T & nitrogen (as ammonia) & 50 & $\mathrm{ug} / \mathrm{L}$ & $\bar{U}$ & $4 / 5 / 95$ \\
\hline PAR 3T & ortho-PO4 & 25 & $u g / L$ & $\mathrm{U}$ & $4 / 5 / 95$ \\
\hline PAR 3T & phosphate, total & 50 & $\mathrm{ug} / \mathrm{L}$ & $U$ & $4 / 5 / 95$ \\
\hline PAR 3T & chlorophyll-a & 3.47 & $\mathrm{mg} / \mathrm{m}^{3}$ & JV & $4 / 27 / 95$ \\
\hline PAR 3T & nitrate & 18.7 & $\mu \mathrm{g} / \mathrm{L}$ & $\mathrm{J}$ & $4 / 27 / 95$ \\
\hline PAR 3T & nitrogen (Kjeldahl) & 420 & $\mu \mathrm{g} / \mathrm{L}$ & & $4 / 27 / 95$ \\
\hline PAR 3T & nitrogen (as ammonia) & 50 & $\mu \mathrm{g} / \mathrm{L}$ & 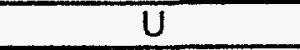 & $4 / 27 / 95$ \\
\hline PAR 3T & ortho-PO4 & 25 & $\mu \mathrm{g} / \mathrm{L}$ & UJ & $4 / 27 / 95$ \\
\hline PAR 3T & phospahte, total & 50 & $\mu \mathrm{g} / \mathrm{L}$ & $J$ & $4 / 27 / 95$ \\
\hline PAR 3T & chlorophyll-a & 0.5 & $\mathrm{mg} / \mathrm{m}^{3}$ & UJ & $5 / 11 / 95$ \\
\hline PAR 3T & nitrate & 5 & $\mu g / L$ & UJ & $5 / 11 / 95$ \\
\hline PAR 3T & nitrogen (Kjeldahl) & 210 & $\mu g / L$ & & $5 / 11 / 95$ \\
\hline PAR 3T & nitrogen (as ammonia) & 50 & $\mu g / L$ & $\bar{U}$ & $5 / 11 / 95$ \\
\hline PAR 3T & ortho-PO4 & 25 & $\mu g / L$ & UJ & $5 / 11 / 95$ \\
\hline PAR 3T & phosphate, total & 320 & $\mu g / L$ & & $5 / 11 / 95$ \\
\hline PAR 3T & chlorophyll-a & 0.5 & $\mathrm{mg} / \mathrm{m}^{3}$ & UJ & $5 / 25 / 95$ \\
\hline PAR 3T & nitrate & 5 & $\mu g / L$ & UJ & $5 / 25 / 95$ \\
\hline
\end{tabular}


Table B-5 (continued). Sample location: Par 3 top (T).

\begin{tabular}{|c|c|c|c|c|c|}
\hline WSRC ID & Analyte & Result & Units & Result qualifier & Sampling date \\
\hline - & 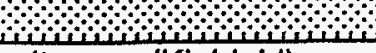 & Hiijis & inisi & क स & 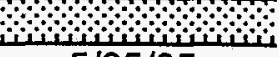 \\
\hline PAR 3T & nitrogen (Kjeldahl) & 360 & $\mu \mathrm{g} / \mathrm{L}$ & & $5 / 25 / 95$ \\
\hline PAR 3T & nitrogen (as ammonia) & 50 & $\mu g / L$ & $\bar{U}$ & $5 / 25 / 95$ \\
\hline PAR 3T & ortho-PO4 & 25 & $\mu g / L$ & UJV & $5 / 25 / 95$ \\
\hline PAR 3T & phosphate, total & 40 & $\mu \mathrm{g} / \mathrm{L}$ & $\mathrm{J}$ & $5 / 25 / 95$ \\
\hline PAR 3T & chlorophyll-a & 6.2 & $\mathrm{mg} / \mathrm{m}^{3}$ & $\mathrm{~J}$ & $6 / 8 / 95$ \\
\hline PAR 3T & nitrate & 5 & $\mu \mathrm{g} / \mathrm{L}$ & UJ & $6 / 8 / 95$ \\
\hline PAR 3T & nitrogen (Kjeldahl) & 50 & $\mu \mathrm{g} / \mathrm{L}$ & $\mathrm{U}$ & $6 / 8 / 95$ \\
\hline PAR 3T & nitrogen (as ammonia) & 50 & $\mu \mathrm{g} / \mathrm{L}$ & $\mathrm{U}$ & $6 / 8 / 95$ \\
\hline PAR 3T & ortho-PO4 & 27.99 & $\mu g / L$ & $\bar{U}$ & $6 / 8 / 95$ \\
\hline PAR 3T & phosphate, total & 50 & $\mu \mathrm{g} / \mathrm{L}$ & $\bar{U}$ & $6 / 8 / 95$ \\
\hline PAR 3T & chlorophyll-a & 4.6 & $\mathrm{mg} / \mathrm{m}^{3}$ & $\mathrm{~J}$ & $6 / 28 / 95$ \\
\hline PAR 3T & nitrate & 5 & $\mu \mathrm{g} / \mathrm{L}$ & UJ & $6 / 28 / 95$ \\
\hline PAR 3T & nitrogen (Kjeldahl) & 420 & $\mu \mathrm{g} / \mathrm{L}$ & & $6 / 28 / 95$ \\
\hline PAR 3T & nitrogen (as ammonia) & 50 & $\mu \mathrm{g} / \mathrm{L}$ & $\mathrm{U}$ & $6 / 28 / 95$ \\
\hline PAR 3T & ortho-PO4 & 25 & $\mu \mathrm{g} / \mathrm{L}$ & $\mathrm{U}$ & $6 / 28 / 95$ \\
\hline PAR 3T & phosphate, total & 50 & $\mu \mathrm{g} / \mathrm{L}$ & $U$ & $6 / 28 / 95$ \\
\hline PAR 3T & chlorophyll-a & 25.3 & $\mathrm{mg} / \mathrm{m}^{3}$ & & $7 / 12 / 95$ \\
\hline PAR 3T & nitrate & 5 & $\mu \mathrm{g} / \mathrm{L}$ & $\mathrm{U}$ & $7 / 12 / 95$ \\
\hline PAR 3T & nitrogen (Kjeldahl) & 590 & $\mu \mathrm{g} / \mathrm{L}$ & & $7 / 12 / 95$ \\
\hline PAR 3T & nitrogen (as ammonia) & 50 & $\mu \mathrm{g} / \mathrm{L}$ & $\bar{U}$ & $7 / 12 / 95$ \\
\hline PAR 3T & ortho-PO4 & 20.3 & $\mu g / L$ & $\bar{U}$ & $7 / 12 / 95$ \\
\hline PAR 3T & phosphate, total & 30 & $\mu g / L$ & 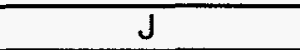 & $7 / 12 / 95$ \\
\hline PAR 3T & chlorophyll-a & 22 & $\mathrm{mg} / \mathrm{m}^{3}$ & & $7 / 26 / 95$ \\
\hline PAR 3T & nitrate & 5 & $\mu \mathrm{g} / \mathrm{L}$ & $\bar{U}$ & $7 / 26 / 95$ \\
\hline PAR 3T & nitrogen (Kjeldahl) & 630 & $\mu \mathrm{g} / \mathrm{L}$ & & $7 / 26 / 95$ \\
\hline PAR 3T & nitrogen (as ammonia) & 50 & $\mu \mathrm{g} / \mathrm{L}$ & $\bar{U}$ & $7 / 26 / 95$ \\
\hline PAR 3T & ortho-PO4 & 25 & $\mu \mathrm{g} / \mathrm{L}$ & $\bar{U}$ & $7 / 26 / 95$ \\
\hline PAR 3T & phosphate, total & 50 & $\mu \mathrm{g} / \mathrm{L}$ & $\bar{U}$ & $7 / 26 / 95$ \\
\hline PAR 3T & chlorophyll-a & 9.63 & $\mathrm{mg} / \mathrm{m}^{3}$ & & $8 / 23 / 95$ \\
\hline PAR 3T & nitrate & 5 & $\mu \mathrm{g} / \mathrm{L}$ & $U$ & $8 / 23 / 95$ \\
\hline PAR 3T & nitrogen (Kjeldahl) & 900 & $\mu g / L$ & & $8 / 23 / 95$ \\
\hline PAR 3T & nitrogen (as ammonia) & 50 & $\mu \mathrm{g} / \mathrm{L}$ & $U$ & $8 / 23 / 95$ \\
\hline PAR 3T & ortho-PO4 & 25 & $\mu g / L$ & $\mathrm{U}$ & $8 / 23 / 95$ \\
\hline PAR 3T & phosphate, total & 110 & $\mu \mathrm{g} / \mathrm{L}$ & & $8 / 23 / 95$ \\
\hline PAR 3T & chlorophyll-a & 6.06 & $\mathrm{mg} / \mathrm{m}^{3}$ & $J$ & $9 / 7 / 95$ \\
\hline PAR 3T & nitrate & 5 & $\mu \mathrm{g} / \mathrm{L}$ & $U$ & $9 / 7 / 95$ \\
\hline PAR 3T & nitrogen (Kjeldahl) & 370 & $\mu \mathrm{g} / \mathrm{L}$ & & $9 / 7 / 95$ \\
\hline PAR 3T & nitrogen (as ammonia) & 50 & $\mu \mathrm{g} / \mathrm{L}$ & $\bar{U}$ & $9 / 7 / 95$ \\
\hline PAR 3T & ortho-PO4 & 25 & $\mu \mathrm{g} / \mathrm{L}$ & $U$ & $9 / 7 / 95$ \\
\hline PAR 3T & phosphate, total & 50 & $\mu \mathrm{g} / \mathrm{L}$ & $\bar{U}$ & $9 / 7 / 95$ \\
\hline
\end{tabular}


Table B-6. Sample location: Par 3 bottom (B).

\begin{tabular}{|c|c|c|c|c|c|}
\hline WSRC ID & Analyte & Result & Units & Result qualifier & Sampling date \\
\hline \multicolumn{6}{|c|}{ 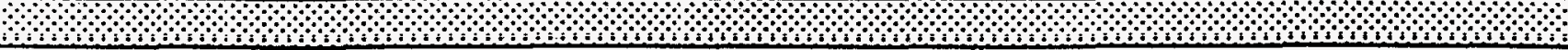 } \\
\hline PAR 3B & chlorophyll-a & 5.18 & $\mathrm{mg} / \mathrm{m}^{3}$ & & $2 / 2 / 95$ \\
\hline PAR 3B & nitrate & 5 & $\mu \mathrm{g} / \mathrm{L}$ & UJ & $2 / 2 / 95$ \\
\hline PAR 3B & nitrogen (Kjeldahl) & 418 & $\mu \mathrm{g} / \mathrm{L}$ & & $2 / 2 / 95$ \\
\hline PAR 3B & nitrogen (as ammonia) & 61.1 & $\mu \mathrm{g} / \mathrm{L}$ & $J$ & $2 / 2 / 95$ \\
\hline PAR 3B & ortho-PO4 & 611 & $\mu \mathrm{g} / \mathrm{L}$ & & $2 / 2 / 95$ \\
\hline PAR 3B & phosphate, total & 194 & $\mu \mathrm{g} / \mathrm{L}$ & $\mathrm{J}$ & $2 / 2 / 95$ \\
\hline PAR 3B & chlorophyll-a & 6.06 & $\mathrm{mg} / \mathrm{m}^{3}$ & & $2 / 22 / 95$ \\
\hline PAR 3B & nitrate & $\overline{94}$ & $\mu \mathrm{g} / \mathrm{L}$ & & $2 / 22 / 95$ \\
\hline PAR 3B & nitrogen (Kjeldahi) & 269 & $\mu \mathrm{g} / \mathrm{L}$ & & $2 / 22 / 95$ \\
\hline PAR 3B & nitrogen (as ammonia) & 50 & $\mu \mathrm{g} / \mathrm{L}$ & UJ & $2 / 22 / 95$ \\
\hline PAR 3B & ortho-PO4 & 50 & $\mu \mathrm{g} / \mathrm{L}$ & $\bar{U}$ & $2 / 22 / 95$ \\
\hline PAR 3B & phosphate, total & 25 & $\mu \mathrm{g} / \mathrm{L}$ & $\bar{U}$ & $2 / 22 / 95$ \\
\hline PAR 3B & chlorophyll-a & 0.5 & $\mathrm{mg} / \mathrm{m}^{3}$ & $\bar{U}$ & $3 / 8 / 95$ \\
\hline PAR 3B & nitrate & 66 & $\mu \mathrm{g} / \mathrm{L}$ & & $3 / 8 / 95$ \\
\hline PAR 3B & nitrogen (Kjeldahl) & 210 & $\mu \mathrm{g} / \mathrm{L}$ & $\bar{V}$ & $3 / 8 / 95$ \\
\hline PAR 3B & nitrogen (as ammonia) & 50 & $\mu \mathrm{g} / \mathrm{L}$ & $\mathrm{U}$ & $3 / 8 / 95$ \\
\hline PAR 3B & ortho-PO4 & 50 & $\mu \mathrm{g} / \mathrm{L}$ & $\bar{U}$ & $3 / 8 / 95$ \\
\hline PAR 3B & phosphate, total & 11 & $\mu \mathrm{g} / \mathrm{L}$ & $J$ & $3 / 8 / 95$ \\
\hline PAR 3B & chlorophyil-a & 1.38 & $\mathrm{mg} / \mathrm{m}^{3}$ & & $3 / 22 / 95$ \\
\hline PAR 3B & nitrate & 61.67 & $u g / L$ & & $3 / 22 / 95$ \\
\hline PAR 3B & nitrogen (Kjeldahl) & 270 & $\mathrm{ug} / \mathrm{L}$ & $\mathrm{J}$ & $3 / 22 / 95$ \\
\hline PAR 3B & nitrogen (as ammonia) & 50 & $\mathrm{ug} / \mathrm{L}$ & $\mathrm{U}$ & $3 / 22 / 95$ \\
\hline PAR 3B & ortho-PO4 & 20.63 & $\mathrm{ug} / \mathrm{L}$ & & $3 / 22 / 95$ \\
\hline PAR 3B & phosphate, total & 50 & $\mathrm{ug} / \mathrm{L}$ & $\mathrm{U}$ & $3 / 22 / 95$ \\
\hline PAR 3B & chlorophyll-a & 0.5 & $\mathrm{mg} / \mathrm{m}^{3}$ & $\mathrm{U}$ & $4 / 5 / 95$ \\
\hline PAR 3B & nitrate & 55.45 & $u g / L$ & & $4 / 5 / 95$ \\
\hline PAR 3B & nitrogen (Kjeldahl) & 240 & $\mathrm{ug} / \mathrm{L}$ & & $4 / 5 / 95$ \\
\hline PAR 3B & nitrogen (as ammonia) & 50 & $\mathrm{ug} / \mathrm{L}$ & $\bar{U}$ & $4 / 5 / 95$ \\
\hline PAR 3B & ortho-PO4 & 10 & $\mathrm{ug} / \mathrm{L}$ & $\mathrm{J}$ & $4 / 5 / 95$ \\
\hline PAR 3B & phosphate, total & 50 & $\mathrm{ug} / \mathrm{L}$ & $\mathrm{U}$ & $4 / 5 / 95$ \\
\hline PAR 3B & chlorophyll-a & 3.38 & $\mathrm{mg} / \mathrm{m}^{3}$ & JV & $4 / 27 / 95$ \\
\hline PAR 3B & nitrate & 23 & $\mu g / L$ & $J$ & $4 / 27 / 95$ \\
\hline PAR 3B & nitrogen (Kjeldahl) & 470 & $\mu \mathrm{g} / \mathrm{L}$ & & $4 / 27 / 95$ \\
\hline PAR 3B & nitrogen (as ammonia) & 140 & $\mu \mathrm{g} / \mathrm{L}$ & & $4 / 27 / 95$ \\
\hline PAR 3B & ortho-PO4 & 17 & $\mu \mathrm{g} / \mathrm{L}$ & $J$ & $4 / 27 / 95$ \\
\hline PAR 3B & phosphate, total & 70 & $\mu \mathrm{g} / \mathrm{L}$ & $\mathrm{J}$ & $4 / 27 / 95$ \\
\hline PAR 3B & chlorophyll-a & 0.5 & $\mathrm{mg} / \mathrm{m}^{3}$ & UJ & $5 / 11 / 95$ \\
\hline PAR 3B & nitrate & 34.33 & $\mu \mathrm{g} / \mathrm{L}$ & $J$ & $5 / 11 / 95$ \\
\hline PAR 3B & nitrogen (Kjeldahl) & 500 & $\mu \mathrm{g} / \mathrm{L}$ & & $5 / 11 / 95$ \\
\hline PAR 3B & nitrogen (as ammonia) & 50 & $\mu \mathrm{g} / \mathrm{L}$ & $U$ & $5 / 11 / 95$ \\
\hline PAR 3B & ortho-PO4 & 50 & $\mu g / L$ & $J$ & $5 / 11 / 95$ \\
\hline PAR 3B & phosphate, total & 470 & $\mu \mathrm{g} / \mathrm{L}$ & & $5 / 11 / 95$ \\
\hline PAR 3B & chlorophyll-a & 3.2 & $\mathrm{mg} / \mathrm{m}^{3}$ & $J$ & $5 / 25 / 95$ \\
\hline
\end{tabular}


Table B-6. Sample location: Par 3 bottom (B).

\begin{tabular}{|c|c|c|c|c|c|}
\hline WSRC ID & Analyte & Result & Units & Result qualifier & Sampling date \\
\hline & $\because \because \because$ & $\because \cdots$ & m & $\omega_{1} \omega_{0}$ & an \\
\hline PAR 3B & nitrate & 31.9 & $\mu \mathrm{g} / \mathrm{L}$ & $J$ & $5 / 25 / 95$ \\
\hline PAR 3B & nitrogen (Kjeldahl) & 750 & $\mu \mathrm{g} / \mathrm{L}$ & & $5 / 25 / 95$ \\
\hline PAR 3B & nitrogen (as ammonia) & 200 & $\mu \mathrm{g} / \mathrm{L}$ & & $5 / 25 / 95$ \\
\hline PAR 3B & ortho-PO4 & 52 & $\mu \mathrm{g} / \mathrm{L}$ & JV & $5 / 25 / 95$ \\
\hline PAR 3B & phosphate, total & 110 & $\mu \mathrm{g} / \mathrm{L}$ & & $5 / 25 / 95$ \\
\hline PAR 3B & chlorophyll-a & 10.4 & $\mathrm{mg} / \mathrm{m}^{3}$ & $J$ & $6 / 8 / 95$ \\
\hline PAR 3B & nitrate & 10.2 & $\mu \mathrm{g} / \mathrm{L}$ & $J$ & $6 / 8 / 95$ \\
\hline PAR 3B & nitrogen (Kjeldahl) & 420 & $\mu \mathrm{g} / \mathrm{L}$ & & $6 / 8 / 95$ \\
\hline PAR 3B & nitrogen (as ammonia) & 50 & $\mu \mathrm{g} / \mathrm{L}$ & $\bar{U}$ & $6 / 8 / 95$ \\
\hline PAR 3B & ortho-PO4 & 17 & $\mu \mathrm{g} / \mathrm{L}$ & $J$ & $6 / 8 / 95$ \\
\hline PAR 3B & phosphate, total & 40 & $\mu \mathrm{g} / \mathrm{L}$ & $\mathrm{J}$ & $6 / 8 / 95$ \\
\hline PAA 3B & chlorophyll-a & 12.4 & $\mathrm{mg} / \mathrm{m}^{3}$ & $J$ & $6 / 28 / 95$ \\
\hline PAR 3B & nitrate & 12.3 & $\mu \mathrm{g} / \mathrm{L}$ & $J$ & $6 / 28 / 95$ \\
\hline PAR 3B & nitrogen (Kjeldahl) & 580 & $\mu \mathrm{g} / \mathrm{L}$ & & $6 / 28 / 95$ \\
\hline PAR 3B & nitrogen (as ammonia) & 80 & $\mu \mathrm{g} / \mathrm{L}$ & $J$ & $6 / 28 / 95$ \\
\hline PAR 3B & ortho-PO4 & 51 & $\mu \mathrm{g} / \mathrm{L}$ & & $6 / 28 / 95$ \\
\hline PAR 3B & phosphate, total & 170 & $\mu \mathrm{g} / \mathrm{L}$ & & $6 / 28 / 95$ \\
\hline PAR 3B & chlorophyll-a & 21.7 & $\mathrm{mg} / \mathrm{m}^{3}$ & & $7 / 12 / 95$ \\
\hline PAR 3B & nitrate & 11.9 & $\mu \mathrm{g} / \mathrm{L}$ & & $7 / 12 / 95$ \\
\hline PAR 3B & nitrogen (Kjeldahl) & 420 & $\mu \mathrm{g} / \mathrm{L}$ & & $7 / 12 / 95$ \\
\hline PAR 3B & nitrogen (as ammonia) & 110 & $\mu \mathrm{g} / \mathrm{L}$ & & $7 / 12 / 95$ \\
\hline PAR 3B & ortho-PO4 & 32 & $\mu \mathrm{g} / \mathrm{L}$ & $\mathrm{J}$ & $7 / 12 / 95$ \\
\hline PAR 3B & phosphate, total & 40 & $\mu \mathrm{g} / \mathrm{L}$ & $J$ & $7 / 12 / 95$ \\
\hline PAR 3B & chlorophyll-a & 28.7 & $\mathrm{mg} / \mathrm{m}^{3}$ & & $7 / 26 / 95$ \\
\hline PAR 3B & nitrate & 5 & $\mu \mathrm{g} / \mathrm{L}$ & $\bar{U}$ & $7 / 26 / 95$ \\
\hline PAR 3B & nitrogen (Kjeldahl) & 640 & $\mu \mathrm{g} / \mathrm{L}$ & & $7 / 26 / 95$ \\
\hline PAR 3B & nitrogen (as ammonia) & 160 & $\mu g / L$ & & $7 / 26 / 95$ \\
\hline PAR 3B & ortho-PO4 & 36 & $\mu g / L$ & $J$ & $7 / 26 / 95$ \\
\hline PAR 3B & phosphate, total & 50 & $\mu \mathrm{g} / \mathrm{L}$ & $\bar{U}$ & $7 / 26 / 95$ \\
\hline PAR 3B & chlorophyll-a & 13.6 & $\mathrm{mg} / \mathrm{m}^{3}$ & & $8 / 23 / 95$ \\
\hline PAR 3B & nitrate & 5 & $\mu \mathrm{g} / \mathrm{L}$ & U & $8 / 23 / 95$ \\
\hline PAR 3B & nitrogen (Kjeldahi) & 1160 & $\mu g / L$ & & $8 / 23 / 95$ \\
\hline PAR 3B & nitrogen (as ammonia) & 50 & $\mu \mathrm{g} / \mathrm{L}$ & U & $8 / 23 / 95$ \\
\hline PAR 3B & ortho-PO4 & 25 & $\mu \mathrm{g} / \mathrm{L}$ & U & $8 / 23 / 95$ \\
\hline PAR 3B & phosphate, total & 260 & $\mu g / L$ & & $8 / 23 / 95$ \\
\hline PAR 3B & chlorophyll-a & 6.9 & $\mathrm{mg} / \mathrm{m}^{3}$ & $J$ & $9 / 7 / 95$ \\
\hline PAR 3B & nitrate & 5 & $\mu \mathrm{g} / \mathrm{L}$ & $\bar{U}$ & $9 / 7 / 95$ \\
\hline PAR 3B & nitrogen (Kjeldahl) & 470 & $\mu g / L$ & & $9 / 7 / 95$ \\
\hline PAR 3B & nitrogen (as ammonia) & 50 & $\mu \mathrm{g} / \mathrm{L}$ & $\mathrm{U}$ & $9 / 7 / 95$ \\
\hline PAA 3B & ortho-PO4 & 25 & $\mu g / L$ & $\bar{U}$ & $9 / 7 / 95$ \\
\hline PAR 3B & phosphate, total & 50 & $\mu g / L$ & $\bar{U}$ & $9 / 7 / 95$ \\
\hline
\end{tabular}


Table B-7. Sample location: Par 4 top (T).

\begin{tabular}{|c|c|c|c|c|c|}
\hline WSRC ID & Analyte & Result & Units & Result qualifier & Sampling date \\
\hline & n & n & m & nn & 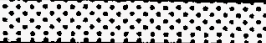 \\
\hline PAR 4T & chlorophyll-a & 0.5 & $\mathrm{mg} / \mathrm{m}^{3}$ & U & $2 / 2 / 95$ \\
\hline PAR 4T & nitrate & 5 & $\mu g / L$ & UJ & $2 / 2 / 95$ \\
\hline PAR 4T & nitrogen (Kjeldahl) & 316.3 & $\mu g / L$ & $\mathrm{~J}$ & $2 / 2 / 95$ \\
\hline PAR 4T & nitrogen (as ammonia) & 33.33 & $\mu g / L$ & & $2 / 2 / 95$ \\
\hline PAR 4T & ortho-PO4 & 50 & $\mu g / L$ & $\mathrm{U}$ & $2 / 2 / 95$ \\
\hline PAR 4T & phosphate, total & 25 & $\mu g / L$ & UJ & $2 / 2 / 95$ \\
\hline PAR 4T & chlorophyll-a & 1.64 & $\mathrm{mg} / \mathrm{m}^{3}$ & & $2 / 22 / 95$ \\
\hline PAR 4T & nitrate & 81 & $\mu g / L$ & & $2 / 22 / 95$ \\
\hline PAR 4T & nitrogen (Kjeldahl) & 381 & $\mu g / L$ & & $2 / 22 / 95$ \\
\hline PAR 4T & nitrogen (as ammonia) & 50 & $\mu g / L$ & UJ & $2 / 22 / 95$ \\
\hline PAR 4T & ortho-PO4 & 50 & $\mu g / L$ & $\bar{U}$ & $2 / 22 / 95$ \\
\hline PAR 4T & phosphate, total & 18.7 & $\mu g / L$ & $J$ & $2 / 22 / 95$ \\
\hline PAR 4T & chlorophyll-a & 0.5 & $\mathrm{mg} / \mathrm{m}^{3}$ & $\bar{U}$ & $3 / 8 / 95$ \\
\hline PAR 4T & nitrate & 33.67 & $\mu g / L$ & & 3/8/95 \\
\hline PAR 4T & nitrogen (Kjeldahl) & 285.68 & $\mu g / L$ & & $3 / 8 / 95$ \\
\hline PAR 4T & nitrogen (as ammonia) & 33.33 & $\mu g / L$ & $\mathrm{U}$ & $3 / 8 / 95$ \\
\hline PAR 4T & ortho-PO4 & 79.31 & $\mu g / L$ & & $3 / 8 / 95$ \\
\hline PAR 4T & phosphate, total & 18.01 & $\mu \mathrm{g} / \mathrm{L}$ & & $3 / 8 / 95$ \\
\hline PAR 4T & chlorophyll-a & 2.99 & $\mathrm{mg} / \mathrm{m}^{3}$ & & $3 / 22 / 95$ \\
\hline PAR 4T & nitrate & 5 & $\mu \mathrm{g} / \mathrm{L}$ & $\underline{U}$ & $3 / 22 / 95$ \\
\hline PAR 4T & nitrogen (Kjeldahl) & 360 & $\mu \mathrm{g} / \mathrm{L}$ & J & $3 / 22 / 95$ \\
\hline PAR 4T & nitrogen (as ammonia) & 50 & $\mu \mathrm{g} / \mathrm{L}$ & $\bar{U}$ & $3 / 22 / 95$ \\
\hline PAR 4T & ortho-PO4 & 25 & $\mu \mathrm{g} / \mathrm{L}$ & $\mathbf{U}$ & $3 / 22 / 95$ \\
\hline PAR 4T & phosphate, total & 50 & $\mu g / L$ & $\mathrm{U}$ & $3 / 22 / 95$ \\
\hline PAR 4T & chlorophyll-a & 0.5 & $\mathrm{mg} / \mathrm{m}^{3}$ & $\bar{U}$ & $4 / 5 / 95$ \\
\hline PAR 4T & nitrate & 5 & $\mu g / L$ & $\mathbf{U}$ & $4 / 5 / 95$ \\
\hline PAR 4T & nitrogen (Kjeldahl) & 350 & $\mu \mathrm{g} / \mathrm{L}$ & & $4 / 5 / 95$ \\
\hline PAR 4T & nitrogen (as ammonia) & 50 & $\mu g / L$ & U & $4 / 5 / 95$ \\
\hline PAR 4T & ortho-PO4 & 25 & $\mu \mathrm{g} / \mathrm{L}$ & $\mathrm{U}$ & $4 / 5 / 95$ \\
\hline PAR 4T & phosphate, total & 50 & $\mu g / L$ & $\mathrm{U}$ & $4 / 5 / 95$ \\
\hline PAR 4T & chlorophyll-a & 3.2 & $\mathrm{mg} / \mathrm{m}^{3}$ & $\mathrm{JV}$ & $4 / 27 / 95$ \\
\hline PAR 4T & nitrate & 18.8 & $\mu \mathrm{g} / \mathrm{L}$ & $\mathrm{J}$ & $4 / 27 / 95$ \\
\hline PAR 4T & nitrogen (Kjeldahl) & 350 & $\mu \mathrm{g} / \mathrm{L}$ & & 4/27/95 \\
\hline PAR 4T & nitrogen (as ammonia) & 50 & $\mu \mathrm{g} / \mathrm{L}$ & $\mathrm{U}$ & $4 / 27 / 95$ \\
\hline PAR 4T & ortho-PO4 & 25 & $\mu \mathrm{g} / \mathrm{L}$ & UJ & $4 / 27 / 95$ \\
\hline PAR 4T & phosphate, total & 36.8 & $\mu \mathrm{g} / \mathrm{L}$ & & $4 / 27 / 95$ \\
\hline PAR 4T & chlorophyll-a & 0.5 & $\mathrm{mg} / \mathrm{m}^{3}$ & UJ & $5 / 11 / 95$ \\
\hline PAR 4T & nitrate & 5 & $\mu \mathrm{g} / \mathrm{L}$ & UJ & $5 / 11 / 95$ \\
\hline PAR 4T & nitrogen (Kjeldahl) & 222.57 & $\mu g / L$ & & $5 / 11 / 95$ \\
\hline PAR 4T & nitrogen (as ammonia) & 33.33 & $\mu \mathrm{g} / \mathrm{L}$ & $\underline{U}$ & $5 / 11 / 95$ \\
\hline PAR 4T & ortho-PO4 & 38.12 & $\mu \mathrm{g} / \mathrm{L}$ & UJ & $5 / 11 / 95$ \\
\hline PAR 4T & phosphate, total & 391.48 & $\mu \mathrm{g} / \mathrm{L}$ & & $5 / 11 / 95$ \\
\hline PAR 4T & chlorophyll-a & 0.5 & $\mathrm{mg} / \mathrm{m}^{3}$ & UJ & $5 / 25 / 95$ \\
\hline PAR 4T & nitrate & 5 & $\mu \mathrm{g} / \mathrm{L}$ & UJ & $5 / 25 / 95$ \\
\hline
\end{tabular}


Table B-7 (continued). Sample location: Par 4 top (T).

\begin{tabular}{|c|c|c|c|c|c|}
\hline WSRC ID & Analyte & Result & Units & Result qualifier & Sampling date \\
\hline & & & & 6 & $\because$ \\
\hline PAR 4T & nitrogen (Kjeldahl) & 285 & $\mu \mathrm{g} / \mathrm{L}$ & J & $5 / 25 / 95$ \\
\hline PAR 4T & nitrogen (as ammonia) & 50 & $\mu \mathrm{g} / \mathrm{L}$ & $\bar{U}$ & $5 / 25 / 95$ \\
\hline PAR 4T & ortho-PO4 & 12.9 & $\mu \mathrm{g} / \mathrm{L}$ & JV & $5 / 25 / 95$ \\
\hline PAR 4T & phosphate, total & 26.67 & $\mu \mathrm{g} / \mathrm{L}$ & $J$ & $5 / 25 / 95$ \\
\hline PAR 4T & chlorophyll-a & 3.2 & $\mathrm{mg} / \mathrm{m}^{3}$ & $\mathrm{~J}$ & $6 / 8 / 95$ \\
\hline PAR 4T & nitrate & 5 & $\mu \mathrm{g} / \mathrm{L}$ & $\mathrm{UJ}$ & $6 / 8 / 95$ \\
\hline PAR 4T & nitrogen (Kjeldahl) & 50 & $\mu \mathrm{g} / \mathrm{L}$ & $\bar{U}$ & $6 / 8 / 95$ \\
\hline PAR 4T & nitrogen (as ammonia) & 50 & $\mu \mathrm{g} / \mathrm{L}$ & $U$ & $6 / 8 / 95$ \\
\hline PAR 4T & ortho-PO4 & 25 & $\mu \mathrm{g} / \mathrm{L}$ & $\mathrm{U}$ & $6 / 8 / 95$ \\
\hline PAR 4T & phosphate, total & 40 & $\mu \mathrm{g} / \mathrm{L}$ & $\mathrm{J}$ & $6 / 8 / 95$ \\
\hline PAR 4T & chlorophyll-a & 15.8 & $\mathrm{mg} / \mathrm{m}^{3}$ & $\mathrm{~J}$ & $6 / 28 / 95$ \\
\hline PAR 4T & nitrate & 5 & $\mu \mathrm{g} / \mathrm{L}$ & UJ & $6 / 28 / 95$ \\
\hline PAR 4T & nitrogen (Kjeldahl) & 420 & $\mu \mathrm{g} / \mathrm{L}$ & & $6 / 28 / 95$ \\
\hline PAR 4T & nitrogen (as ammonia) & 50 & $\mu \mathrm{g} / \mathrm{L}$ & $\bar{U}$ & $6 / 28 / 95$ \\
\hline PAR 4T & ortho-PO4 & 11 & $\mu \mathrm{g} / \mathrm{L}$ & $\mathrm{J}$ & $6 / 28 / 95$ \\
\hline PAR 4T & phosphate, total & 50 & $\mu \mathrm{g} / \mathrm{L}$ & $\bar{U}$ & $6 / 28 / 95$ \\
\hline PAR 4T & chlorophyll-a & 26.1 & $\mathrm{mg} / \mathrm{m}^{3}$ & & $7 / 12 / 95$ \\
\hline PAR 4T & nitrate & 12 & $\mu \mathrm{g} / \mathrm{L}$ & & $7 / 12 / 95$ \\
\hline PAR 4T & nitrogen (Kjeldahl) & 470 & $\mu \mathrm{g} / \mathrm{L}$ & & $7 / 12 / 95$ \\
\hline PAR 4T & nitrogen (as ammonia) & 50 & $\mu \mathrm{g} / \mathrm{L}$ & $\mathrm{U}$ & $7 / 12 / 95$ \\
\hline PAR 4T & ortho-PO4 & 13 & $\mu \mathrm{g} / \mathrm{L}$ & $J$ & $7 / 12 / 95$ \\
\hline PAR 4T & phosphate, total & 50 & $\mu \mathrm{g} / \mathrm{L}$ & $\mathrm{U}$ & $7 / 12 / 95$ \\
\hline PAR 4T & chlorophyll-a & 29.2 & $\mathrm{mg} / \mathrm{m}^{3}$ & & $7 / 26 / 95$ \\
\hline PAR 4T & nitrate & 4.03 & $\overrightarrow{\mu g / L}$ & $\bar{U}$ & $7 / 26 / 95$ \\
\hline PAR 4T & nitrogen (Kjeldahl) & 1020 & $\mu \mathrm{g} / \mathrm{L}$ & & $7 / 26 / 95$ \\
\hline PAR 4T & nitrogen (as ammonia) & 50 & $\mu \mathrm{g} / \mathrm{L}$ & $\bar{U}$ & $7 / 26 / 95$ \\
\hline PAR 4T & ortho-PO4 & 11 & $\mu \mathrm{g} / \mathrm{L}$ & $J$ & $7 / 26 / 95$ \\
\hline PAR 4T & phosphate, total & 50 & $\mu \mathrm{g} / \mathrm{L}$ & $\mathrm{U}$ & $7 / 26 / 95$ \\
\hline PAR 4T & chlorophyll-a & 12.7 & $\mathrm{mg} / \mathrm{m}^{3}$ & & $8 / 23 / 95$ \\
\hline PAR 4T & nitrate & 5 & $\mu \mathrm{g} / \mathrm{L}$ & $\mathrm{U}$ & $8 / 23 / 95$ \\
\hline PAR 4T & nitrogen (Kjeldahl) & 1520 & $\mu \mathrm{g} / \mathrm{L}$ & & $8 / 23 / 95$ \\
\hline PAR 4T & nitrogen (as ammonia) & 30 & $\mu \mathrm{g} / \mathrm{L}$ & $J$ & $8 / 23 / 95$ \\
\hline PAR 4T & ortho-PO4 & 25 & $\mu \mathrm{g} / \mathrm{L}$ & $\bar{U}$ & $8 / 23 / 95$ \\
\hline PAR 4T & phosphate, total & 50 & $\mu \mathrm{g} / \mathrm{L}$ & $\bar{U}$ & $8 / 23 / 95$ \\
\hline PAR 4T & chlorophyll-a & 7.77 & $\mathrm{mg} / \mathrm{m}^{3}$ & $J$ & $9 / 7 / 95$ \\
\hline PAR 4T & nitrate & 5 & $\mu \mathrm{g} / \mathrm{L}$ & $\mathrm{U}$ & $9 / 7 / 95$ \\
\hline PAR 4T & nitrogen (Kjeldahl) & 350 & $\mu \mathrm{g} / \mathrm{L}$ & & $9 / 7 / 95$ \\
\hline PAR 4T & nitrogen (as ammonia) & 50 & $\mu \mathrm{g} / \mathrm{L}$ & $U$ & $9 / 7 / 95$ \\
\hline PAR 4T & ortho-PO4 & 25 & $\mu \mathrm{g} / \mathrm{L}$ & $\mathrm{U}$ & $9 / 7 / 95$ \\
\hline PAR 4T & phosphate, total & 50 & $\mu \mathrm{g} / \mathrm{L}$ & $\mathrm{U}$ & $9 / 7 / 95$ \\
\hline
\end{tabular}


Table B-8. Sample location: Par 4 bottom (B).

\begin{tabular}{|c|c|c|c|c|c|}
\hline WSRC ID & Analyte & Result & Units & Result qualifier & Sampling date \\
\hline hl一 & hl一 & . & h & $\because \because$ & hl一 \\
\hline PAR 4B & chlorophyll-a & 0.5 & $\mathrm{mg} / \mathrm{m}^{3}$ & $\mathrm{U}$ & $2 / 2 / 95$ \\
\hline PAR 4B & nitrate & 5 & $\mu \mathrm{g} / \mathrm{L}$ & UJ & $2 / 2 / 95$ \\
\hline PAR 4B & nitrogen (Kjeldahl) & 1160 & $\mu \mathrm{g} / \mathrm{L}$ & & $2 / 2 / 95$ \\
\hline PAR 4B & nitrogen (as ammonia) & 50 & $\mu \mathrm{g} / \mathrm{L}$ & $\bar{U}$ & $2 / 2 / 95$ \\
\hline PAR 4B & ortho-PO4 & 274 & $\mu \mathrm{g} / \mathrm{L}$ & & $2 / 2 / 95$ \\
\hline PAR 4B & phosphate, total & 25 & $\mu \mathrm{g} / \mathrm{L}$ & $\overline{\mathrm{UJ}}$ & $2 / 2 / 95$ \\
\hline PAR 4B & chlorophyll-a & 0.5 & $\mathrm{mg} / \mathrm{m}^{3}$ & $\bar{U}$ & $2 / 22 / 95$ \\
\hline PAR 4B & nitrate & 85 & $\mu \mathrm{g} / \mathrm{L}$ & & $2 / 22 / 95$ \\
\hline PAR 4B & nitrogen (Kjeldahl) & 308 & $\mu \mathrm{g} / \mathrm{L}$ & & $2 / 22 / 95$ \\
\hline PAR 4B & nitrogen (as ammonia) & 50 & $\mu \mathrm{g} / \mathrm{L}$ & $\mathrm{UJ}$ & $2 / 22 / 95$ \\
\hline PAR 4B & ortho-PO4 & 50 & $\mu \mathrm{g} / \mathrm{L}$ & $\mathrm{U}$ & $2 / 22 / 95$ \\
\hline PAR 4B & phosphate, total & 12 & $\mu \mathrm{g} / \mathrm{L}$ & $\mathrm{J}$ & $2 / 22 / 95$ \\
\hline PAR 4B & chlorophyll-a & 0.5 & $\mathrm{mg} / \mathrm{m}^{3}$ & $\bar{U}$ & $3 / 8 / 95$ \\
\hline PAR 4B & nitrate & 67 & $\mu \mathrm{g} / \mathrm{L}$ & & $3 / 8 / 95$ \\
\hline PAR 4B & nitrogen (Kjeldahl) & 247 & $\mu \mathrm{g} / \mathrm{L}$ & & $3 / 8 / 95$ \\
\hline PAR 4B & nitrogen (as ammonia) & 50 & $\mu \mathrm{g} / \mathrm{L}$ & $\bar{U}$ & $3 / 8 / 95$ \\
\hline PAR 4B & ortho-PO4 & 50 & $\mu \mathrm{g} / \mathrm{L}$ & $\bar{U}$ & $3 / 8 / 95$ \\
\hline PAR 4B & phosphate, total & 10 & $\mu \mathrm{g} / \mathrm{L}$ & $\mathrm{J}$ & $3 / 8 / 95$ \\
\hline PAR 4B & nitrate & 67 & $\mu \mathrm{g} / \mathrm{L}$ & & $3 / 22 / 95$ \\
\hline PAR 4B & nitrogen (Kjeldahl) & 230 & $\mu \mathrm{g} / \mathrm{L}$ & $\overline{V J}$ & $3 / 22 / 95$ \\
\hline PAR 4B & nitrogen (as ammonia) & 50 & $\mu \mathrm{g} / \mathrm{L}$ & $\bar{U}$ & $3 / 22 / 95$ \\
\hline PAR 4B & ortho-PO4 & 11 & $\mu \mathrm{g} / \mathrm{L}$ & $\mathrm{J}$ & $3 / 22 / 95$ \\
\hline PAR 4B & phosphate, total & 50 & $\mu \mathrm{g} / \mathrm{L}$ & $\bar{U}$ & $3 / 22 / 95$ \\
\hline PAR 4B & chlorophyll-a & 0.5 & $\mathrm{mg} / \mathrm{m}^{3}$ & $\bar{U}$ & $4 / 5 / 95$ \\
\hline PAR 4B & nitrate & 84.1 & $\mu \mathrm{g} / \mathrm{L}$ & & $4 / 5 / 95$ \\
\hline PAR 4B & nitrogen (Kjeldahl) & 380.2 & $\mu g / L$ & & $4 / 5 / 95$ \\
\hline PAR 4B & nitrogen (as ammonia) & 50 & $\mu \mathrm{g} / \mathrm{L}$ & $\bar{U}$ & $4 / 5 / 95$ \\
\hline PAR 4B & ortho-PO4 & 72 & $\mu \mathrm{g} / \mathrm{L}$ & & $4 / 5 / 95$ \\
\hline PAR 4B & phosphate, total & 55.14 & $\mu \mathrm{g} / \mathrm{L}$ & & $4 / 5 / 95$ \\
\hline PAR 4B & chlorophyll-a & 4.62 & $\mathrm{mg} / \mathrm{m}^{3}$ & JV & $4 / 27 / 95$ \\
\hline PAR 4B & nitrate & 26 & $\mu \mathrm{g} / \mathrm{L}$ & $J$ & $4 / 27 / 95$ \\
\hline PAR 4B & nitrogen (Kjeldahl) & 760 & $\mu \mathrm{g} / \mathrm{L}$ & & $4 / 27 / 95$ \\
\hline PAR 4B & nitrogen (as ammonia) & 190 & $\mu \mathrm{g} / \mathrm{L}$ & & $4 / 27 / 95$ \\
\hline PAR 4B & ortho-PO4 & 45 & $\mu \mathrm{g} / \mathrm{L}$ & $J$ & $4 / 27 / 95$ \\
\hline PAR 4B & phosphate, total & 70 & $\mu \mathrm{g} / \mathrm{L}$ & $J$ & $4 / 27 / 95$ \\
\hline PAR 4B & chlorophyll-a & 0.5 & $\mathrm{mg} / \mathrm{m}^{3}$ & UJ & $5 / 11 / 95$ \\
\hline PAR 4B & nitrate & 21.6 & $\mu \mathrm{g} / \mathrm{L}$ & $J$ & $5 / 11 / 95$ \\
\hline PAR 4B & nitrogen (Kjeldahl) & 590 & $\mu \mathrm{g} / \mathrm{L}$ & & $5 / 11 / 95$ \\
\hline PAR 4B & nitrogen (as ammonia) & 50 & $\mu \mathrm{g} / \mathrm{L}$ & $\bar{U}$ & $5 / 11 / 95$ \\
\hline PAR 4B & ortho-PO4 & 47 & $\mu \mathrm{g} / \mathrm{L}$ & $\mathrm{J}$ & $5 / 11 / 95$ \\
\hline PAR 4B & phosphate, total & 160 & $\mu g / L$ & & $5 / 11 / 95$ \\
\hline PAR 4B & chlorophyll-a & 0.5 & $\mathrm{mg} / \mathrm{m}^{3}$ & UJ & $5 / 25 / 95$ \\
\hline PAR 4B & nitrate & 5 & $\mu g / L$ & UJ & $5 / 25 / 95$ \\
\hline PAR 4B & nitrogen (Kjeldahl) & 770 & $\mu \mathrm{g} / \mathrm{L}$ & & $5 / 25 / 95$ \\
\hline
\end{tabular}


Table B-8 (continued). Sample location: Par 4 bottom (B).

\begin{tabular}{|c|c|c|c|c|c|}
\hline WSRC ID & Analyte & Result & Units & Result qualifier & Sampling date \\
\hline & & & & & $5 / 25 / 95$ \\
\hline PAR 4B & nitrogen (as ammonia) & 320 & $\mu \mathrm{g} / \mathrm{L}$ & & $5 / 25 / 95$ \\
\hline PAR 4B & ortho-PO4 & 62 & $\mu \mathrm{g} / \mathrm{L}$ & JV & $5 / 25 / 95$ \\
\hline PAR 4B & phosphate, total & 150 & $\mu \mathrm{g} / \mathrm{L}$ & & $5 / 25 / 95$ \\
\hline PAR 4B & nitrate & 13.1 & $\mu g / L$ & $J$ & $6 / 8 / 95$ \\
\hline PAR 4B & nitrogen (Kjeldahl) & 850 & $\mu \mathrm{g} / \mathrm{L}$ & & $6 / 8 / 95$ \\
\hline PAR 4B & nitrogen (as ammonia) & 310 & $\mu \mathrm{g} / \mathrm{L}$ & & $6 / 8 / 95$ \\
\hline$\overline{P A R ~ 4 B}$ & ortho-PO4 & 125 & $\mu \mathrm{g} / \mathrm{L}$ & & $6 / 8 / 95$ \\
\hline PAR 4B & phosphate, total & 218.24 & $\mu \mathrm{g} / \mathrm{L}$ & & $6 / 8 / 95$ \\
\hline PAR 4B & chlorophyll-a & 19.8 & $\mathrm{mg} / \mathrm{m}^{3}$ & J & $6 / 28 / 95$ \\
\hline PAR 4B & nitrate & 10.1 & $\mu \mathrm{g} / \mathrm{L}$ & $\mathrm{J}$ & $6 / 28 / 95$ \\
\hline$\overline{P A R ~ 4 B}$ & nitrogen (Kjeldahl) & 580 & $\mu \mathrm{g} / \mathrm{L}$ & & $6 / 28 / 95$ \\
\hline PAR 4B & nitrogen (as ammonia) & 60 & $\mu g / L$ & $J$ & $6 / 28 / 95$ \\
\hline PAR 4B & ortho-PO4 & 40 & $\mu \mathrm{g} / \mathrm{L}$ & J & $6 / 28 / 95$ \\
\hline PAR 4B & phosphate, total & 50 & $\mu \mathrm{g} / \mathrm{L}$ & $\mathrm{J}$ & $6 / 28 / 95$ \\
\hline PAR 4B & chlorophyll-a & 22.7 & $\mathrm{mg} / \mathrm{m}^{3}$ & & $7 / 12 / 95$ \\
\hline PAR 4B & nitrate & 3.33 & $\mu g / L$ & $\bar{U}$ & $7 / 12 / 95$ \\
\hline PAR 4B & nitrogen (Kjeldahl) & 210 & $\mu \mathrm{g} / \mathrm{L}$ & & $7 / 12 / 95$ \\
\hline PAR 4B & nitrogen (as ammonia) & 50 & $\mu \mathrm{g} / \mathrm{L}$ & $\bar{U}$ & $7 / 12 / 95$ \\
\hline PAR 4B & ortho-PO4 & 23 & $\mu \mathrm{g} / \mathrm{L}$ & $\mathrm{J}$ & $7 / 12 / 95$ \\
\hline PAR 4B & phosphate, total & 30 & $\mu \mathrm{g} / \mathrm{L}$ & $J$ & $7 / 12 / 95$ \\
\hline PAR 4B & chlorophyll-a & 23.8 & $\mathrm{mg} / \mathrm{m}^{3}$ & & $7 / 26 / 95$ \\
\hline PAR 4B & nitrate & 5 & $\mu \mathrm{g} / \mathrm{L}$ & $\mathrm{U}$ & $7 / 26 / 95$ \\
\hline PAR 4B & nitrogen (Kjeldahl) & 980 & $\mu \mathrm{g} / \mathrm{L}$ & & $7 / 26 / 95$ \\
\hline PAR 4B & nitrogen (as ammonia) & 50 & $\mu \mathrm{g} / \mathrm{L}$ & $\bar{U}$ & $7 / 26 / 95$ \\
\hline PAR 4B & ortho-PO4 & 25 & $\mu \mathrm{g} / \mathrm{L}$ & $\bar{U}$ & $7 / 26 / 95$ \\
\hline PAR 4B & phosphate, total & 50 & $\mu \mathrm{g} / \mathrm{L}$ & $\bar{U}$ & $7 / 26 / 95$ \\
\hline PAR 4B & chlorophyll-a & 10.9 & $\mathrm{mg} / \mathrm{m}^{3}$ & & $8 / 23 / 95$ \\
\hline PAR 4B & nitrate & 5 & $\mu \mathrm{g} / \mathrm{L}$ & $\bar{U}$ & $8 / 23 / 95$ \\
\hline PAR 4B & nitrogen (Kjeldahl) & 1920 & $\mu \mathrm{g} / \mathrm{L}$ & & $8 / 23 / 95$ \\
\hline PAR 4B & nitrogen (as ammonia) & 50 & $\mu \mathrm{g} / \mathrm{L}$ & $\mathrm{J}$ & $8 / 23 / 95$ \\
\hline PAR 4B & ortho-PO4 & 25 & $\mu \mathrm{g} / \mathrm{L}$ & $\bar{U}$ & $8 / 23 / 95$ \\
\hline PAR 4B & phosphate, total & 50 & $\mu \mathrm{g} / \mathrm{L}$ & $\bar{U}$ & $8 / 23 / 95$ \\
\hline PAR 4B & chlorophyll-a & 23.4 & $\mathrm{mg} / \mathrm{m}^{3}$ & $J$ & $9 / 7 / 95$ \\
\hline PAR 4B & nitrate & 5 & $\mu \mathrm{g} / \mathrm{L}$ & $\bar{U}$ & $9 / 7 / 95$ \\
\hline PAR 4B & nitrogen (Kjeldahl) & 890 & $\mu \mathrm{g} / \mathrm{L}$ & & $9 / 7 / 95$ \\
\hline PAR 4B & nitrogen (as ammonia) & 420 & $\mu \mathrm{g} / \mathrm{L}$ & & $9 / 7 / 95$ \\
\hline PAR 4B & ortho-PO4 & 25 & $\mu \mathrm{g} / \mathrm{L}$ & $\mathrm{U}$ & $9 / 7 / 95$ \\
\hline PAR 4B & phosphate, total & 50 & $\mu \mathrm{g} / \mathrm{L}$ & $\mathrm{U}$ & 9/7/95 \\
\hline
\end{tabular}

